\title{
Data on Ground-Water Quality, Carson Valley and Topaz Lake areas, Douglas County, Nevada, For Year Ending September 1986
}

By Carl E. Thodal

\section{U.S. GEOLOGICAL SURVEY}

Open-File Report 88-453

Prepared in cooperation with DOUGLAS COUNTY

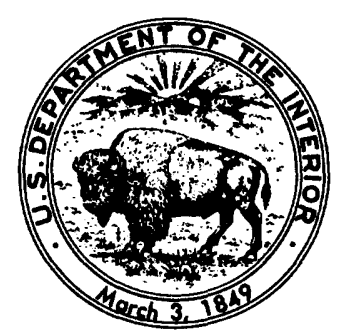

Carson City, Nevada 1989 


\title{
DEPARTMENT OF THE INTERIOR
}

MANUEL LUJAN, JR., Secretary

\author{
U.S. GEOLOGICAL SURVEY \\ Dallas L. Peck, Director
}

For additional information write to:

U.S. Geological Survey Room 227, Federal Building 705 North Plaza Street Carson City, NV 89701
Copies of this report may be purchased from:

U.S. Geological Survey Books and Open-File Reports Section Federal Center, Building 810

Box 25425

Denver, CO 80225 
Abstract

Introduction

Methods and materials used

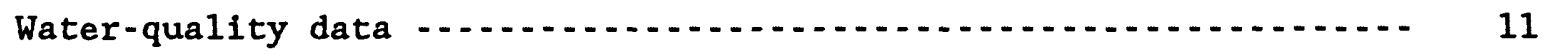

Data tabulations

References cited

\section{ILLUSTRATIONS}

Figure 1. Map showing geographic features of Douglas County, and approximate areal extent of figures 2 and $5 \ldots \ldots . . .3$

2. Maps showing monitoring-we11 locations $\ldots \ldots$

3-5. Maps showing monitoring we11s where:

3. Primary drinking-water standards were exceeded .... 20

4. Secondary drinking-water standards were exceeded -.. 21

5. Organic compounds were detected by gaschromatography and flame-ionization analysis ...... 22 


\section{TABLES}

Page

Table 1 . Information regarding wells used in the

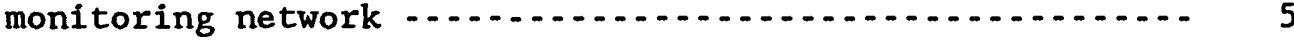

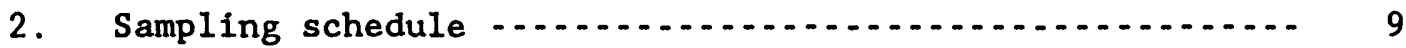

3. Sample containers and field treatments

for laboratory analyses ......................... 10

4. Background information on constituents and

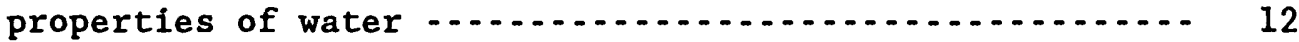

5-10. Water-quality data:

5. Physical properties and major chemical constituents - 26

6. Dissolved nitrogen, phosphorus, and organic carbon -. 38

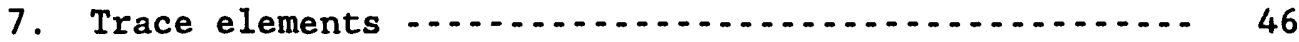

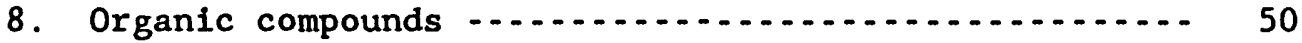

9. Dissolved radium-226, radon-222, and uranium ...... 51

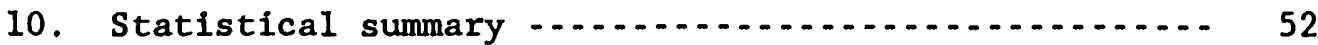


"Inch-pound" units of measure used in this report may be converted to metric (International System) units by using the following factors:

\section{Multiply}

Acre-foot (acre-ft)

Foot $(f t)$

Inch (in.)

$\operatorname{Mile}(\mathrm{mi})$

Square mile $\left(\mathrm{mi}^{2}\right)$
By

1,233

0.3048

25.40

1.609

2.590

\section{To obtain}

Cubic meter $\left(\mathrm{m}^{3}\right)$

Meter (m)

Millimeter $(\mathrm{mm})$

Kilometer $(\mathrm{km})$

Square kilometer $\left(\mathrm{km}^{2}\right)$

For temperature, degrees Celsius $\left({ }^{\circ} \mathrm{C}\right)$ may be converted to degrees Fahrenheit $\left({ }^{\circ} \mathrm{F}\right)$ by using the formula ${ }^{\circ} \mathrm{F}=\left[(1.8)\left({ }^{\circ} \mathrm{C}\right)\right]+32$.

SEA LEVEL

In this report, "sea level" refers to the National Geodetic Vertical Datum of 1929 (NGVD of 1929), which is derived from a general adjustment of the first-order leveling networks of both the United States and Canada (formerly called "Sea-Level Datum of 1929"). 


\title{
DATA ON GROUND-WATER QUALITY, CARSON VALLEY AND TOPAZ LAKE AREAS, DOUGLAS COUNTY, NEVADA, FOR YEAR ENDING SEPTEMBER 1986
}

By Carl E. Thodal

\begin{abstract}
Douglas County encompasses 708 square miles in western Nevada and has been one of the fastest growing counties in the Nation, according to the 1980 census. This rapid population growth has led to concern about the present and future impacts of development upon the ground water, which is the principal source of drinking water for most of Douglas County. In 1985, the U.S. Geological Survey, in cooperation with the Douglas County Department of Public Works, established a network of 33 wells in the Carson Valley and Topaz Lake areas to characterize the current ground-water quality and its seasonal variability and to monitor temporal responses to changing land-use activities. This report presents data collected from that network during November 1985 through September 1986. The primary drinking-water standard was exceeded for fluoride $(4.0 \mathrm{mg} / \mathrm{L}$ [milligrams per liter]) at one observation well and for nitrate-nitrogen (10 $\mathrm{mg} / \mathrm{L}$ ) at one domestic well. The primary drinking-water standard for arsenic ( $50 \mu \mathrm{g} / \mathrm{L}$ [micrograms per liter]) was exceeded in one observation well. Secondary drinking-water standards were exceeded as follows: $\mathrm{pH}$ (6.5-8.5 units) at one public-supply well and one domestic well, sulfate $(250 \mathrm{mg} / \mathrm{L})$ at one observation well, dissolved solids $(500 \mathrm{mg} / \mathrm{L})$ at two observation wells, iron $(600 \mu \mathrm{g} / \mathrm{L})$ at one observation well, and manganese $(50 \mu \mathrm{g} / \mathrm{L})$ at three observation wells and one public-supply well. The presence and overall concentration (but not the individual identities) of organic compounds were estimated using a gas chromatograph and flame-ionization detector for ground-water samples collected from 30 network wells. Seven wells (two domestic and five observation) had overall concentrations greater than 10 micrograms per liter relative to the internal standard compound perdeuteronaphthalene.
\end{abstract}




\section{INTRODUCTION}

Douglas County encompasses $708 \mathrm{mi}^{2}$ (U.S. Bureau of the Census, 1983, p. 82) in western Nevada (figure 1). The county is bounded on the west by Lake Tahoe, on the east by the Pine Nut Mountains, and on the south by the Wellington Hills. The north-south trending mountain ranges divide the county into three major valleys: the Lake Tahoe Basin along the western border (about $70 \mathrm{mi}^{2}$ ), Carson Valley in the west-central portion (about $420 \mathrm{mi}^{2}$ ), and the Nevada portion of Antelope Valley in the southeastern apex of the county (about $220 \mathrm{mi}^{2}$ ). Two major river systems flow in a northerly direction through the county: the Carson River through Carson Valley and the West Walker River through Antelope Valley. The economics of the county are dominated by agriculture in Carson and Antelope Valleys, and by tourism and gaming along the southeast shore of Lake Tahoe. The major population centers are Stateline at Lake Tahoe and the MindenGardnerville area in Carson Valley.

Douglas County has been one of the fastest growing counties in the Nation, with a 182-percent increase in population between the 1970 and 1980 censuses (Governor's Office of Planning Coordination, 1984, page 37). This increase is apparently a result of recent economic growth in the gaming industry, availability of residential property, and the aesthetic qualities of the area. This rapid growth has led to concern about the present and future impacts of development on the land and water resources.

Ground water is the principal source of drinking water for most of Douglas County except in the Lake Tahoe basin where the lake is the primary source. Ground water is also used for irrigation in Carson and Antelope Valleys. In 1969, just prior to the rapid population increase, the estimated 6,000 inhabitants in Douglas County withdrew approximately 7,000 acre-feet of ground water for irrigation and about 420 acre-feet for public supply. By 1985, the County's population had increased to 23,200 inhabitants and estimated annual ground-water withdrawals had increased to 10,500 acre-feet for irrigation and 3,900 acre-feet for public supply. Estimates of surface-water withdrawals decreased from 240,000 acre-feet for irrigation in 1969 to 220,000 acre-feet in 1985, while surface-water withdrawals for public supply increased from 1,200 acre-feet in 1969 to about 2,200 acre-feet in 1985 (Smales and Harrill, 1971 , p. 18; E. A. Frick, U.S. Geological Survey, written communication, 1987). 


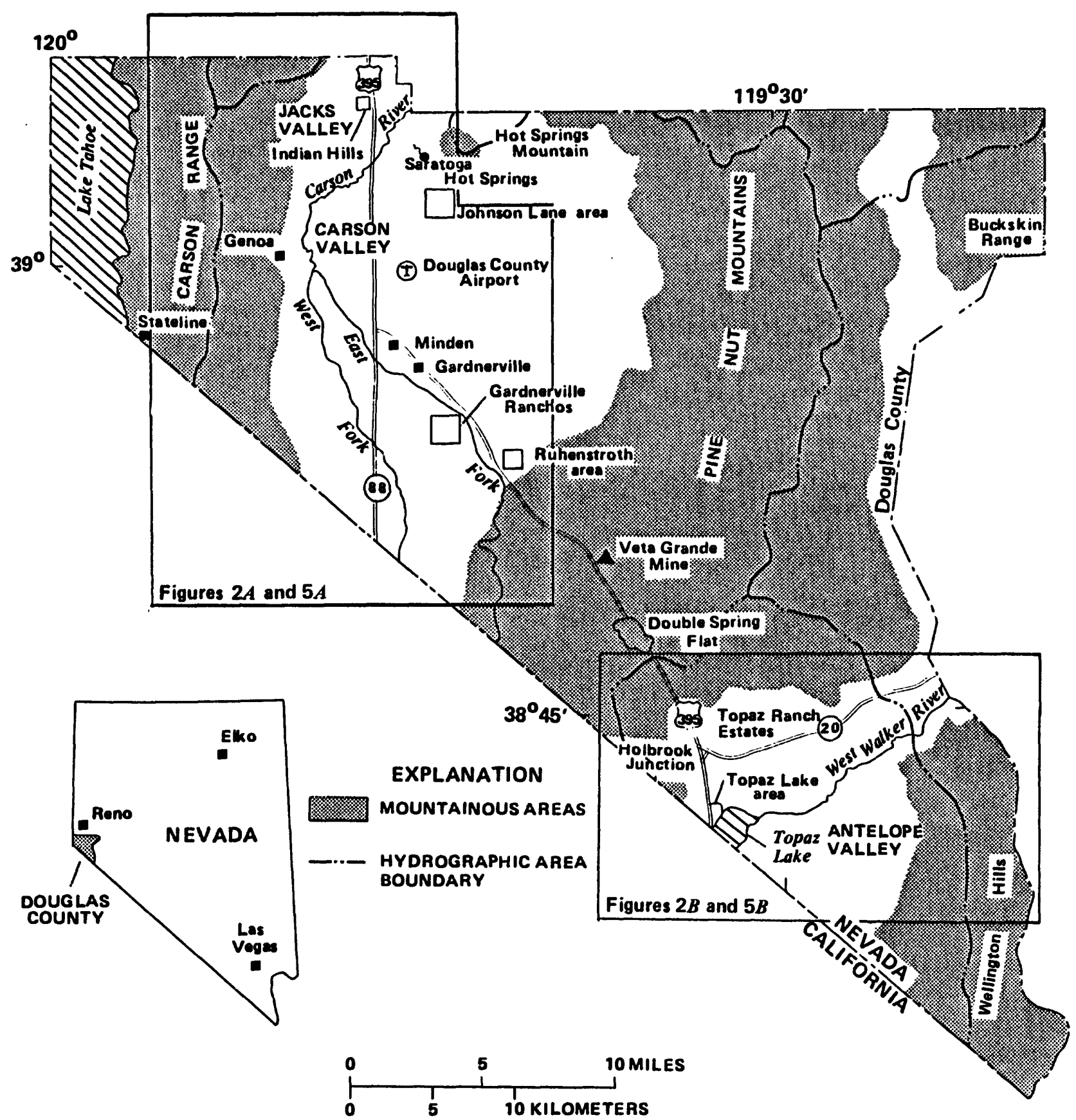

FIGURE 1.-Geographic features of Douglas County and approximate areal extent of figures 2 and 5. 
Six community water-supply systems of varying sizes serve developed areas in and near the Minden-Gardnerville area in southern Carson Valley and a residential area in Indian Hills in the northern part of the valley. Smaller community systems serve developments adjacent to and northeast of Topaz Lake in Antelope Valley. The remainder of the county outside the Lake Tahoe basin is served by private domestic wells.

Potential sources of ground-water contamination in Douglas County include (1) natural, localized sources of mineralized water and of uranium and its radioactive daughter products (Otton and others, 1985); (2) a gold mining and milling operation in the southeastern corner of Carson Valley; (3) a large regional landfill in Carson Valley; (4) infiltration of nutrients from the use of agricultural fertilizers in Carson and Antelope Valleys; (5) land application of municipal sewage effluents in Carson Valley; (6) percolation of domestic wastes from private septic tanks in more densely developed suburban areas of the county (Garcia, 1989); and (7) localized contamination by improper disposal of organic solvents and leakage of petroleum products from underground storage tanks. Potentially adverse health effects of organic compounds, the increasing reliance on land application for disposal of municipal sewage effluents, and percolation of domestic wastes are the principal water-quality concerns for maintenance of the public water supply. Currently, local communities in Carson Valley operate two land disposal systems, and sewage sludge and effluents are imported for land disposal from sewage-treatment systems in the Lake Tahoe basin. Increased use of land application in Carson Valley is being considered to expand the sewage-treatment facilities.

Previous studies have identified areas where degradation of groundwater quality may occur in Douglas County (Nowlin, 1982; Garcia, 1989). In addition to the ground-water quality analysis, a three-dimensional model was developed to predict surface-water outflow and ground-water levels in response to various land-use alternatives (Maurer, 1986). Actual measurements of ground-water levels and estimates of pumpage have been compiled from ongoing data-collection efforts related to ground-water resources in Carson Valley (Berger, 1987).

In 1985, the U.S. Geological Survey, in cooperation with the Douglas County Department of Public Works, established a network of 33 wells for monitoring ground-water quality in the Carson Valley and Topaz Lake areas. Several of the monitoring wells are not used for domestic purposes and only five represent public supply sources. The monitoring wells are identified and described in table 1 and locations are shown in figure 2. 
TABLE 1.--Information regarding wells used in monitoring network

["-- Indicates that no Information was avallable]

U.S. Geological Survey site designations

\begin{tabular}{|c|c|c|c|}
\hline $\begin{array}{c}\text { Site } \\
\text { number } 1 \\
\text { (f1gure 2) }\end{array}$ & $\begin{array}{l}\text { Type } \\
\text { of } \\
\text { site }\end{array}$ & $\begin{array}{c}\text { Standard } \\
\text { Identif Ication }\end{array}$ & $\begin{array}{l}\text { Local } \\
\text { Identification }\end{array}$ \\
\hline
\end{tabular}

Depth (feet

below land surface)

\begin{tabular}{|c|c|c|c|c|}
\hline & & & & \\
\hline $\begin{array}{c}\text { altitude } \\
\text { (feet above } \\
\text { sea level) }\end{array}$ & We 11 & $\begin{array}{c}\text { Top of } \\
\text { open } \\
\text { Interval }\end{array}$ & $\begin{array}{l}\text { Bottom } \\
\text { of open } \\
\text { Interval }\end{array}$ & $\begin{array}{l}\text { Casing } \\
\text { diameter } \\
\text { (inches) }\end{array}$ \\
\hline
\end{tabular}

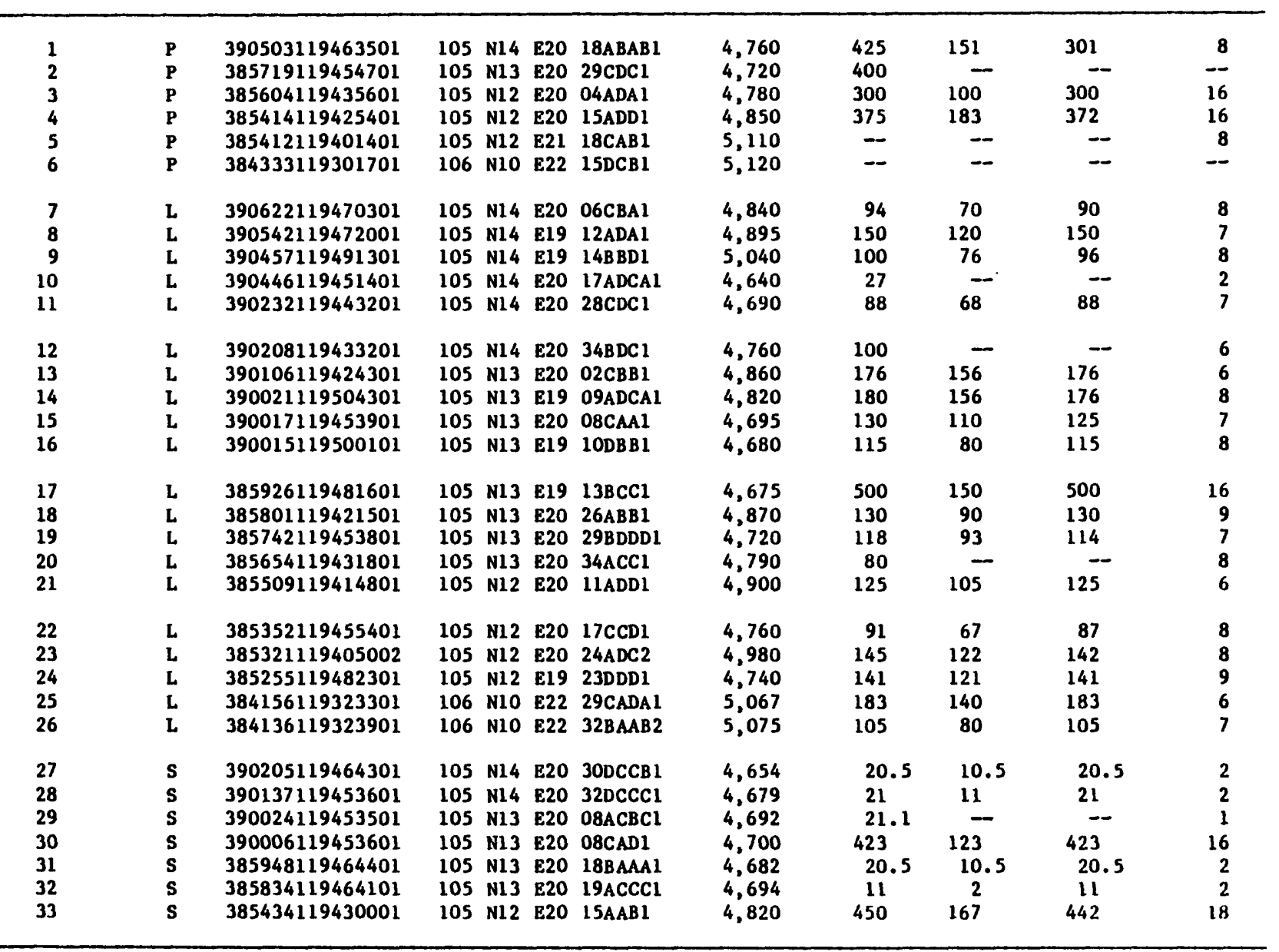

1 In this table, we11s are identified by a short site number, and by the standard identification (ID), and the local (Nevada) ID used by the U.S. Geological Survey. Except in this table, only the short site numbers (1-33) are used, for convenience.

The standard Geological Survey site ID is based on the grid system of latitude and longitude. The ID indicates the geographic location of each site, and provides a unique number for each. The ID consists of 15 digits: The first 6 denote the degrees, minutes, and seconds of latitude; the next 7 denote degrees, minutes, and seconds of longitude; and the last 2 digits (assigned sequentially) identify the sites within a l-second grid. For example, site 385604119435601 is at $38^{\circ} 56^{\prime} 04^{\circ}$ lat 1 tude and $119^{\circ} 43^{\prime} 56^{\circ}$ " long 1 tude, and it 18 the first site recorded in that $1-8$ econd grid.

The local well-identification system 18 based on an index of hydrographic areas in Nevada (Rush, 1968) and the rectangular subdivision of the public lands referenced to the Mount Diablo base line and meridian. Each designation consists of four units separated by spaces: The first unit is the hydrographic area number. The second unit 18 the township, preceded by an $N$ or $S$ to indicate location north or south of the base line. The third unit 18 the range, preceded by an $E$ to indicate location east of the meridian. The fourth unit consists of the section number and letters designating the quarter section, quarter-quarter section, and 80 on (A, B, C, and D Indicate the northeast, northwest, southwest, and southeast quarters, respectively), followed by number indicating the sequence in which the well was recorded. For example, well $105 \mathrm{~N} 12$ E21 18CABI 18 in Carson Valley (hydrographic area 105). It is the first well recorded in the Wht of the NEt of the SWt of section 18, Township 12 North, Range 21 Bast, Mount Diablo base 1 ine and meridian.

2 P, primary; L, long-term term; S, supplemental. 


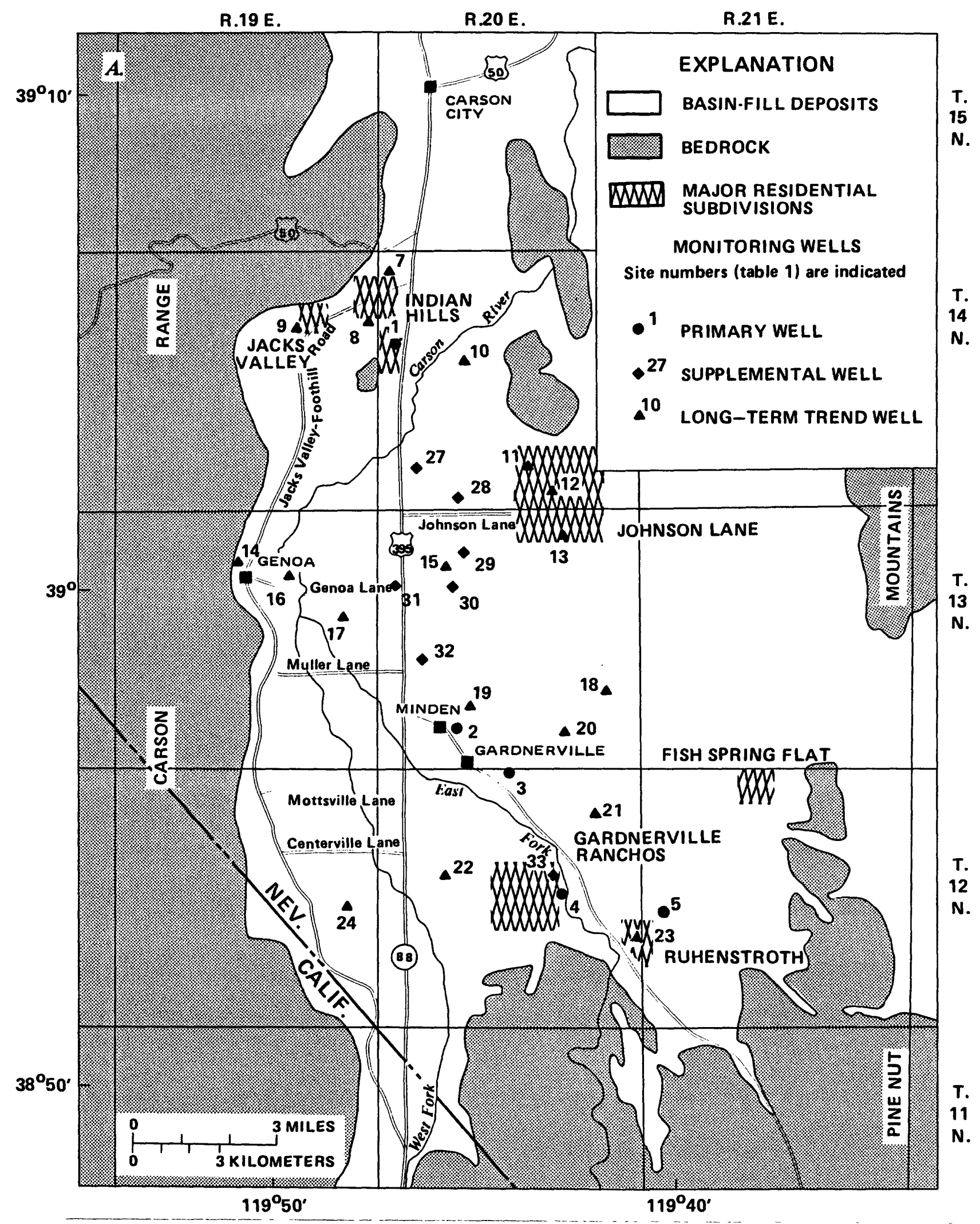

FIGURE 2.-Monitoring-well locations in (A) Carson Valley and (B) Topaz Lake area. 


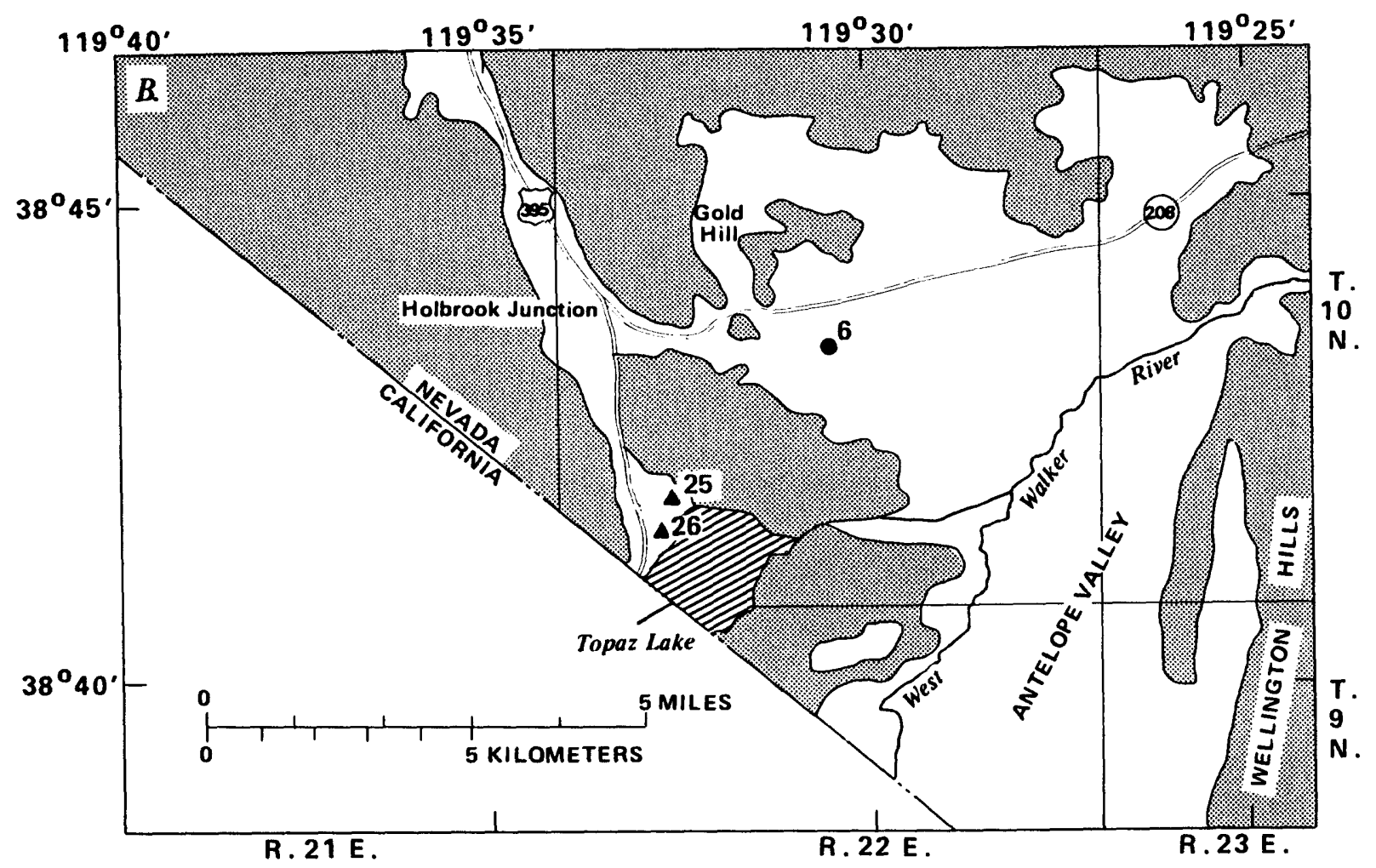

FIGURE 2.-Continued.

The purpose of this report is to present the ground-water quality data collected during November 1985 through September 1986. The data include: physical properties and major chemical constituents; nutrients; trace elements; methylene-chloride-extractable organic compounds; and radionuclides. The frequency of data collection was every other month for 6 "primary" sites, every third month for 20 "long-term trend" sites, and once for 7 "supplemental" sites. The data will serve as a baseline to establish current ground-water quality conditions in the study area and to characterize their seasonal variability. This report will also assist in the assessment of future ground-water quality responses to changing land-use practices. 
The monitoring network is composed of wells defined as (1) primary sites-heavily pumped municipal wells or wells in areas with a high potential for contamination; (2) long-term trend sites--wells that will indicate time trends in water-quality; and (3) supplemental sites--wells added on a one-time basis each year to fill in data gaps. The network consists of 6 primary sites, 20 long-term trend-sites, and 7 supplemental sites (table 1, figure 2). Five shallow (less than 30 feet deep) observation wells, installed by the Geological Survey, were selected as supplemental sites in order to characterize the chemical quality of the watertable aquifer in Carson Valley. The sampling schedule is listed in table 2 .

Four supplemental sites (numbers 27, 28, 31, and 32) and long-term trend site number 10 are constructed of polyvinyl chloride (PVC) thermoplastic well casings. PVC has many desirable properties for monitoringwell construction. Some data, however, suggest potential interferences from the use of PVC for monitoring trace chemical species (Barcelona and others, 1983, page 43) and particularly when casing sections and screens are joined by solvent primers and cements (Barcelona and others, page 41). The integrity of major chemical data collected from these sites to characterize the water quality of the shallow ground-water system should not be affected by the materials used for well construction, but interpretation of trace chemical data (for example, organic compounds and trace metals) warrants careful scrutiny.

Water-quality variables measured at each site included (1) temperature, by hand thermometer, accurate to $\pm 0.5^{\circ} \mathrm{C}$; (2) $\mathrm{pH}$, by digital field instrument, accurate to \pm 0.1 unit; (3) specific conductance, by field instrument, accurate to \pm 5 microsiemens per centimeter $(\mu \mathrm{s} / \mathrm{cm})$; and (4) alkalinity, by incremental$\mathrm{pH}$ titration with a field instrument, accurate to \pm 1 milligram per liter $(\mathrm{mg} / \mathrm{L}) \mathrm{CaCO}_{3}$. Field instruments were calibrated immediately before each use according to the methods described by the U.S. Environmental Protection Agency (1972, chapters 3 and 4) and by Barnes (1964).

Ground-water samples were collected using the existing pump in each monitoring well, or from the natural discharge from flowing wells. Samples from Geological Survey observation wells and we11 number 105 N14 E20 17ADCA1 were obtained with a peristaltic pump. Each well was allowed to discharge a minimum volume equivalent to the calculated volume of three well bores in an attempt to collect samples which are representative of the aquifer. Water temperature, $\mathrm{pH}$, and specific-conductance measurements were made frequently during this flushing period. 
TABLE 2.--Sampling schedule

[P, primary sites; L, long-term trend sites; S, supplemental sites; $Z$, selected sites; --, no sampling scheduled]

\begin{tabular}{|c|c|c|c|c|c|c|c|c|}
\hline Constituent or property & $\begin{array}{l}\text { Nov. } \\
1985\end{array}$ & $\begin{array}{l}\text { Ja n } \\
1986\end{array}$ & $\begin{array}{l}\text { Feb } \\
1986\end{array}$ & $\begin{array}{l}\text { Mar. } \\
1986\end{array}$ & $\begin{array}{l}\text { May } \\
1986\end{array}$ & $\begin{array}{l}\text { July } \\
1986\end{array}$ & $\begin{array}{l}\text { Aug } \\
1986\end{array}$ & $\begin{array}{l}\text { Sep } \\
1986\end{array}$ \\
\hline
\end{tabular}

\section{Field measurements:}

Temperature, $\mathrm{pH}$, specific

$\begin{array}{llllllllll}\text { conductance, and alkalinity } & P, L & P & L & P & P, L & P & L, S & P\end{array}$

Major dissolved constituents:

Bicarbonate-carbonate, calcium,

chloride, fluoride, magnesium,

potassium, silica, sodium,

$\begin{array}{llllllllll}\text { sulfate, and dissolved solids } & P, L & P & L & P & P, L & P & L, S & P\end{array}$

Selected nutrients:

Dissolved nitrogen and

phosphorus compounds and

dissolved organic carbon

$\begin{array}{llllllll}\text { P, L } & \text { P } & \text { L } & \text { P } & \text { P, L } & \text { P } & \text { L, S } & \text { P }\end{array}$

Trace elements:

Dissolved arsenic, barium, boron, cadmium, chromium, copper, Iron, lead, manganese, mercury, selenium, silver, and zinc

Radiochemistry:

Dissolved radium-226,

radon -222 , and uranium

Semiquantitative organic scan:

\begin{tabular}{llllllll}
-- & -- & - & - & $Z$ & $Z$ & - & - \\
- & -- & - & - & $P$ & $\mathrm{~L}, \mathrm{~S}$ & -- & -- \\
\hline
\end{tabular}

Water sampled for laboratory analysis was collected and treated by standard Geological Survey methods (Skougstad and others, 1979) and shipped within 2 days to the U.S. Geological Survey laboratory in Arvada, Colo. The types of containers and field treatment procedures used for the various determinations are listed in table 3 . The methods and precisions of these analyses are described by Feltz and Anthony (1985, part 5). 
TABLE 3.--Sample containers and field treatments for laboratory analyses [Abbreviations: $\mathrm{mL}$, milliliter: ${ }^{\circ} \mathrm{C}$, degrees Celsius]

\begin{tabular}{|c|c|c|}
\hline Type of analysis & Container & Treatment \\
\hline Dissolved major anions & $\begin{array}{l}250-\mathrm{mL} \text { polyethylene } \\
\text { bottle, field-rinsed }\end{array}$ & Filtered ${ }^{1}$ \\
\hline $\begin{array}{l}\text { Dissolved major cations } \\
\text { and trace constituents }\end{array}$ & $\begin{array}{l}500-\mathrm{mL} \text { polyethylene } \\
\text { bottle, acid-rinsed }\end{array}$ & $\begin{array}{l}\text { Filtered, }{ }^{1} \text { acidified } \\
\text { with nitric acid }\end{array}$ \\
\hline Dissolved nutrients & $\begin{array}{l}250-\mathrm{mL} \text { brown } \\
\text { polyethylene bottle, } \\
\text { field-rinsed }\end{array}$ & $\begin{array}{l}\text { Filtered, chilled, } \\
\text { preserved with } \\
\text { mercuric chloride }\end{array}$ \\
\hline Dissolved organic carbon & $\begin{array}{l}125-\mathrm{mL} \text { glass bottle, } \\
\text { baked, } 350^{\circ} \mathrm{C}\end{array}$ & Filtered, ${ }^{2}$ chilled \\
\hline Dissolved mercury & $\begin{array}{l}250-\mathrm{mL} \text { glass bottle, } \\
\text { acid-rinsed }\end{array}$ & $\begin{array}{l}\text { Filtered, acidified } \\
\text { with nitric acid, } \\
\text { preserved with potas- } \\
\text { sium dichromate }\end{array}$ \\
\hline Dissolved radon-222 & $80-\mathrm{mL}$ glass bubbler & None \\
\hline $\begin{array}{l}\text { Semiquantitative } \\
\text { organic scan }\end{array}$ & $\begin{array}{l}\text { 1-liter glass bottle, } \\
\text { baked, } 350^{\circ} \mathrm{C}\end{array}$ & Chilled \\
\hline
\end{tabular}

1 Prerinsed, 0.45 -micrometer-pore-size cellulose-nitrate membrane filter. 2 Prerinsed, 0.45-micrometer-pore-size, silver-metal membrane filter.

A semiquantitative "scan" for the presence and overall concentration (but not the individual identities) of organic substances was made on samples from 30 selected sites using a gas chromatograph and flameionization detector. Gas chromatography (GC) is an analytical technique used to separate a mixture of organic compounds into discrete "GC peaks." Each peak represents an individual organic component of the original mixture. Organic compounds originally dissolved in water must first 
be extracted from aqueous solution into an organic solvent prior to GC analysis. Methylene chloride serves as the organic solvent for this analysis. Each extracted sample is injected into the GC where a flow of inert gas carries it onto a capillary column. The column is heated precisely (from 45 to $300{ }^{\circ} \mathrm{C}$ ) at a controlled rate ( $6{ }^{\circ} \mathrm{C}$ per minute), resulting in separation of the various discrete organic components. As each compound exits the GC, it enters the flame-ionization detector, where a flame ionizes the compound, causing an electrical current to flow in a circuit. The resulting current is proportional to the mass of the ionized compound (H.R. Feltz, U.S. Geological Survey, written communication, 1985).

The estimated overall concentration is calculated relative to the response of the compound perdeuteronaphthalene (which is an internal standard added to each sample just prior to analysis), and is reported as micrograms of equivalent perdeuteronaphthalene per liter of sample. The number of organic compounds detected is determined by the number of peaks on the chromatogram that (1) have an area greater than 10 percent of the added internal standard and (2) are not detected in a blank (deionized water that is treated as a sample in all aspects). This method gives a cost-effective indication of overall organic contamination, providing direction for selective analysis by the more expensive gaschromatographic/mass-spectrometric technique that is necessary to identify specific organic compounds.

Water samples collected from 10 wells selected as a representative subset of the monitoring network, were submitted for laboratory analysis to determine concentrations of dissolved uranium, dissolved radium-226, and dissolved radon-222. Exposure to naturally occurring radionuclides of the uranium-238 decay series, including radon-222 and radium-226, is believed to represent a significant threat to public health (Hobbs and McClellan, 1980, p. 518-522).

\section{WATER-QUALITY DATA}

This report presents the data collected from November 1985 through September 1986. Physical and chemical data are given in tables 5-9 (see section titled "Data Tabulations"), and a statistical summary, by constituent, of water-quality data collected at all wells in the monitoring network, is presented in table 10 ( $p$. 52). Measurements below detection limits were not included when summary statistics were calculated; thus, for some constituents the estimated statistics may be skewed higher than the true population statistics.

Table 4 lists background information on constituents and properties of water, including standards and criteria for water use. 

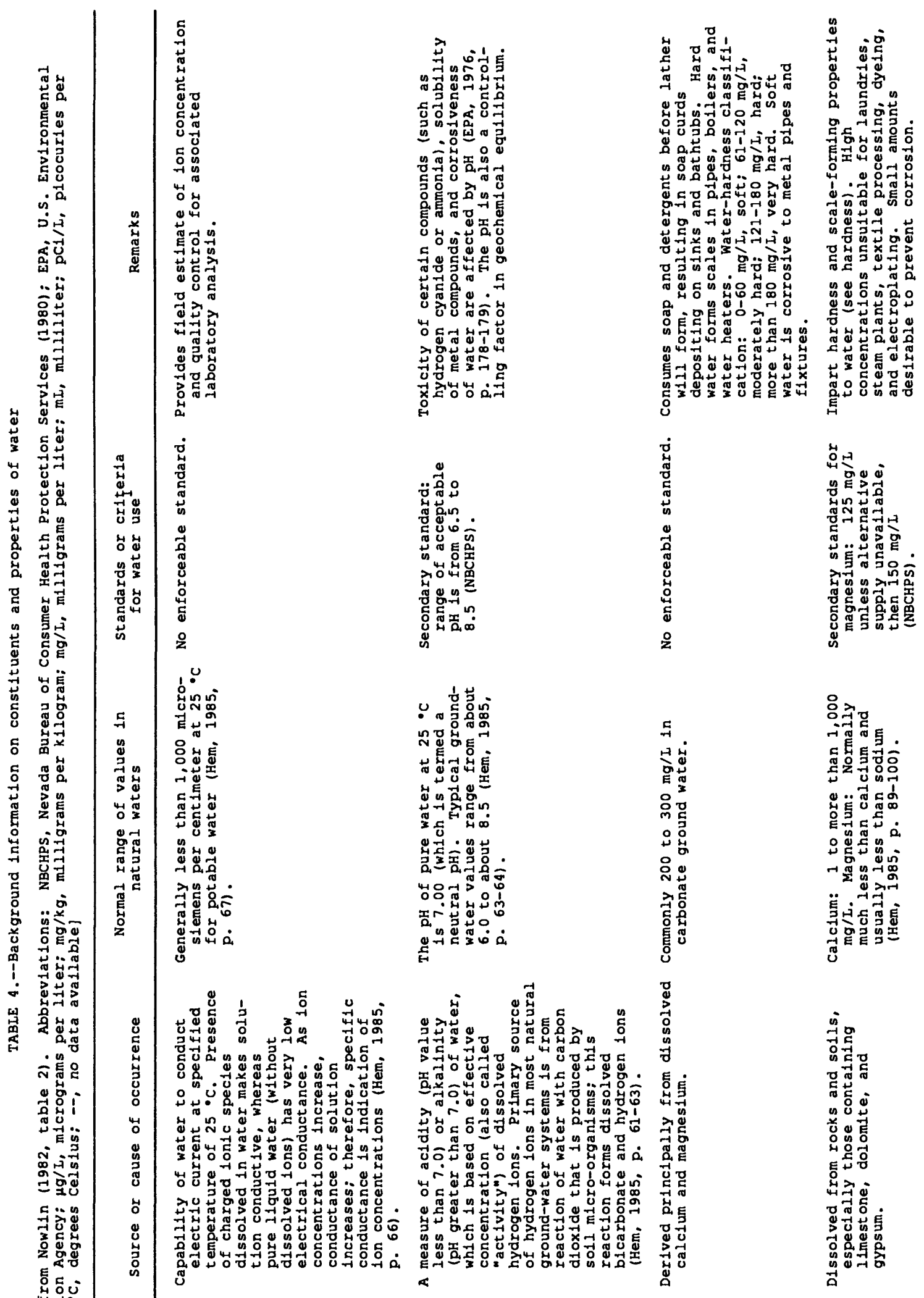

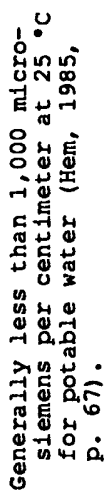

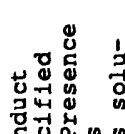

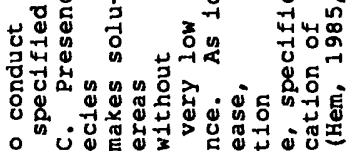

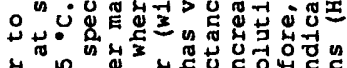

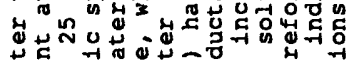

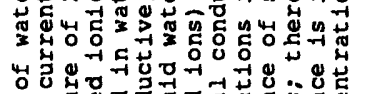

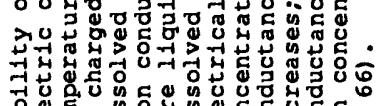

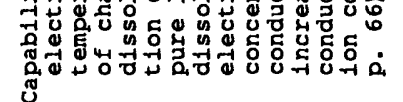

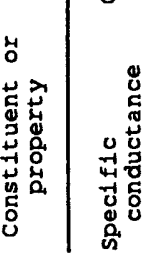

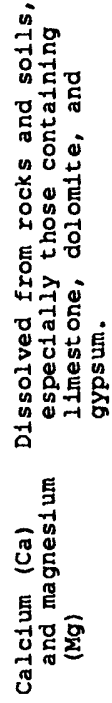



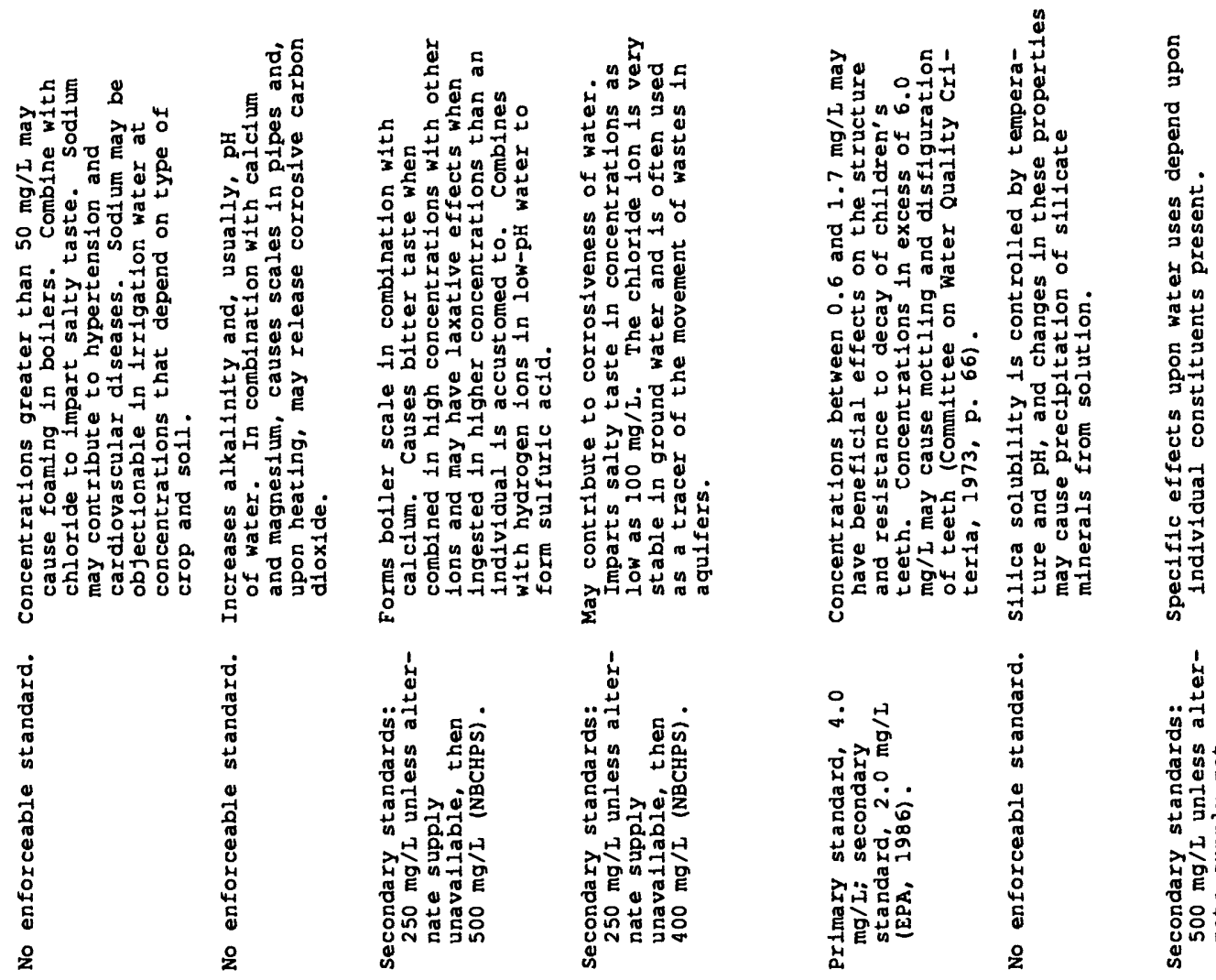

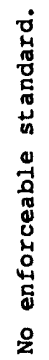
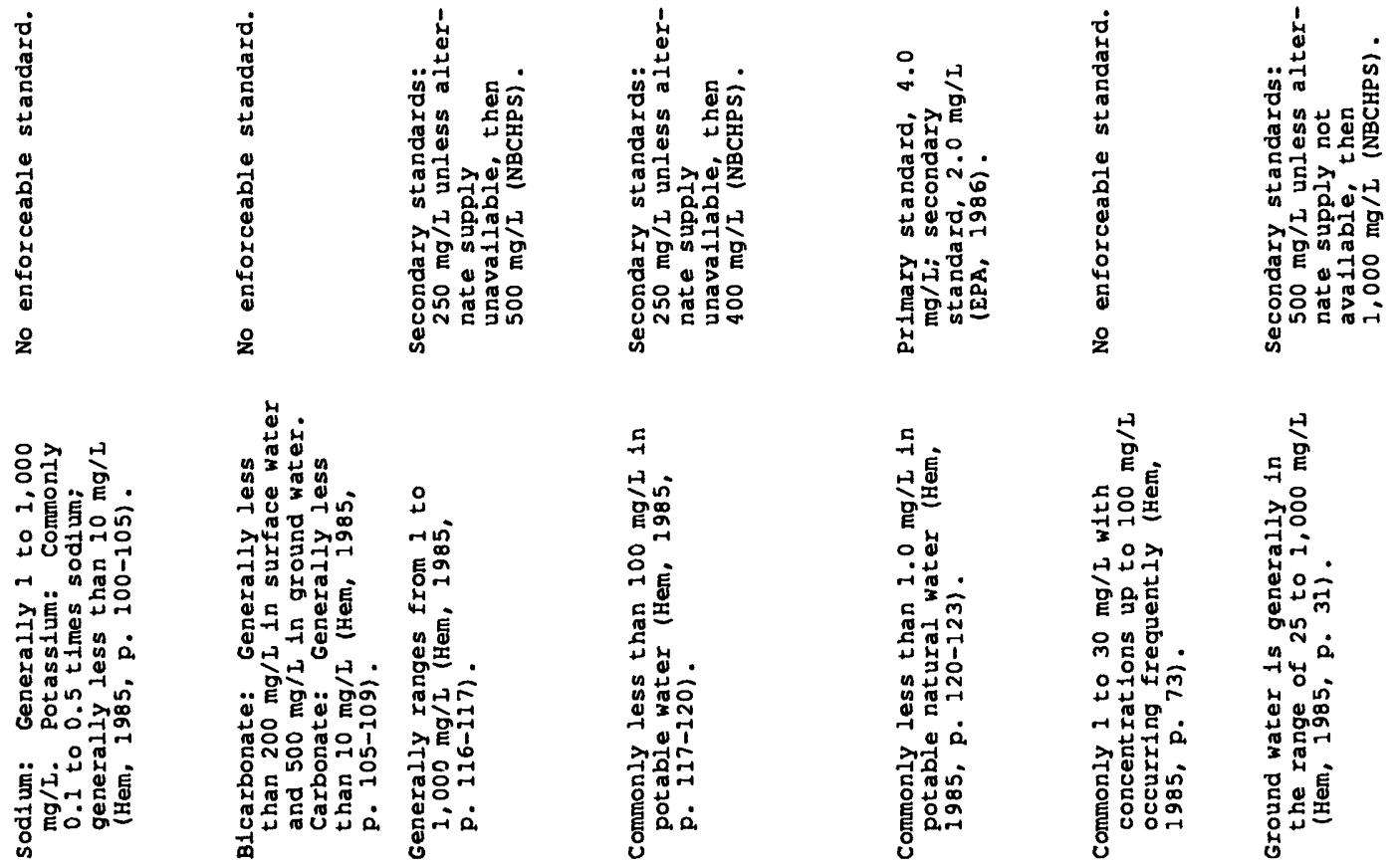

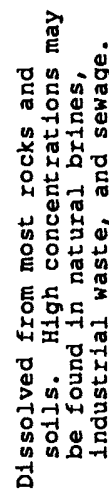
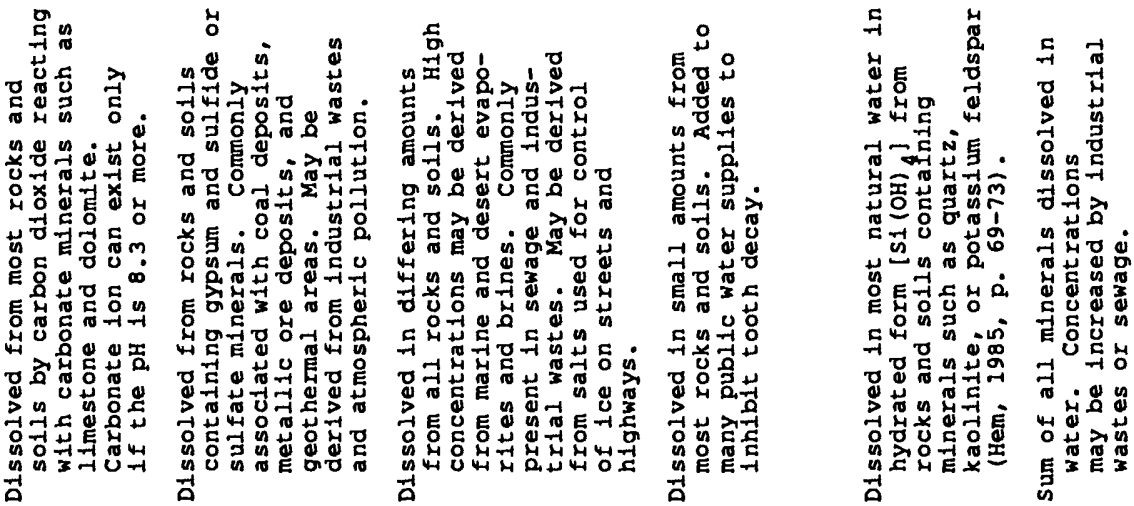

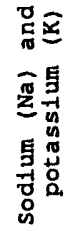
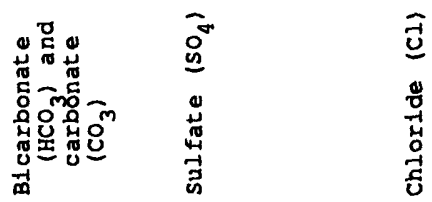

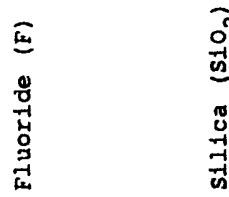

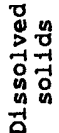




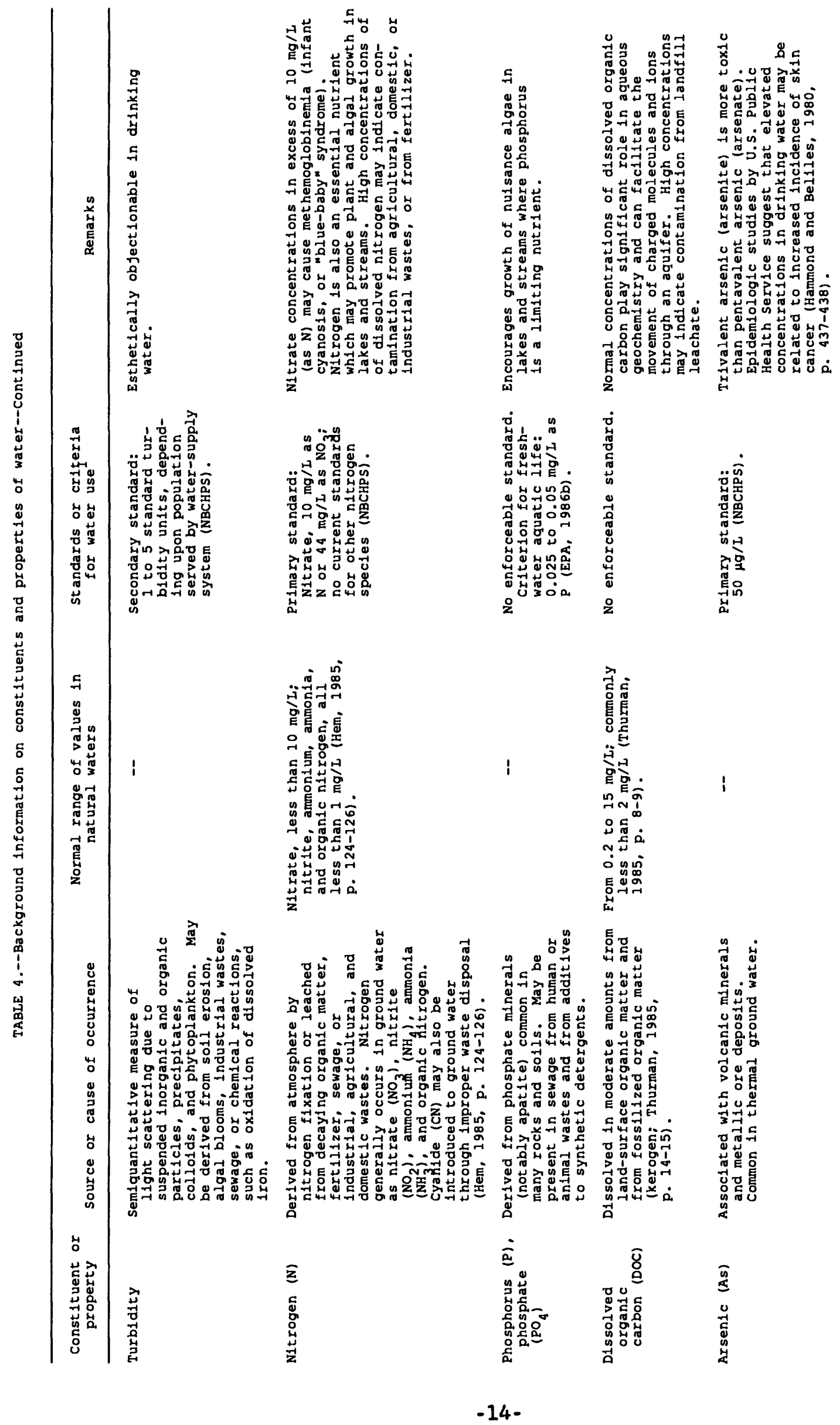



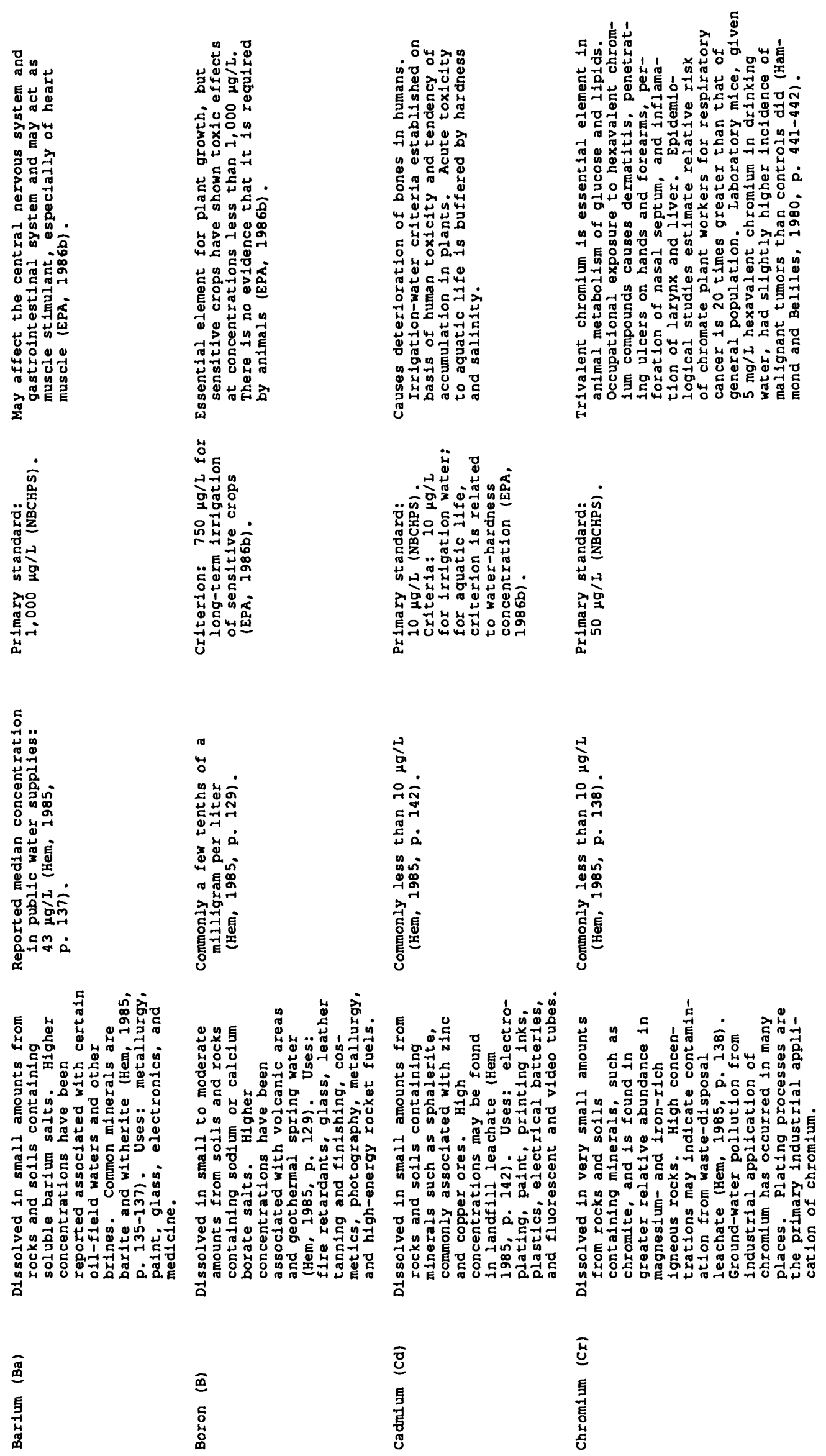


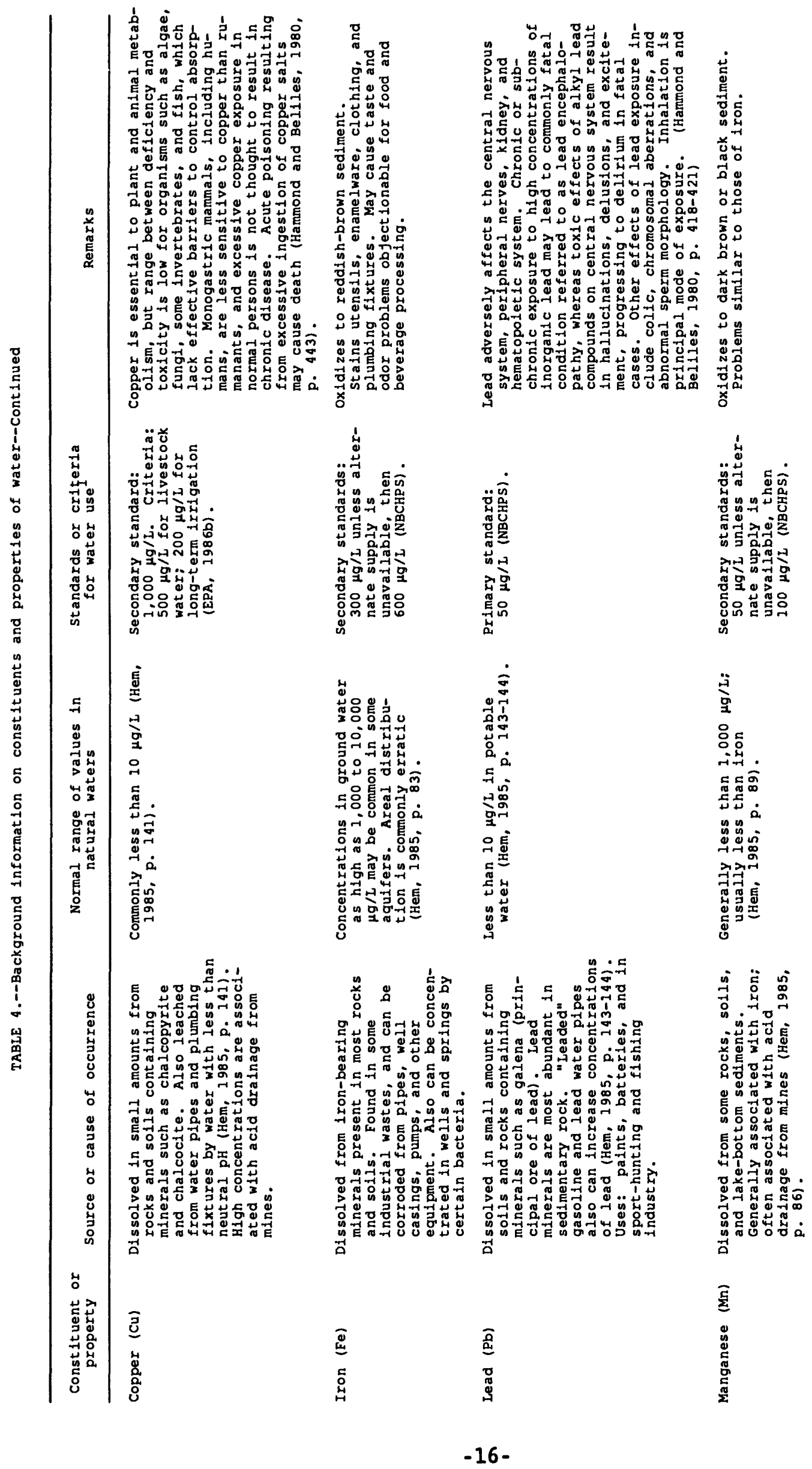



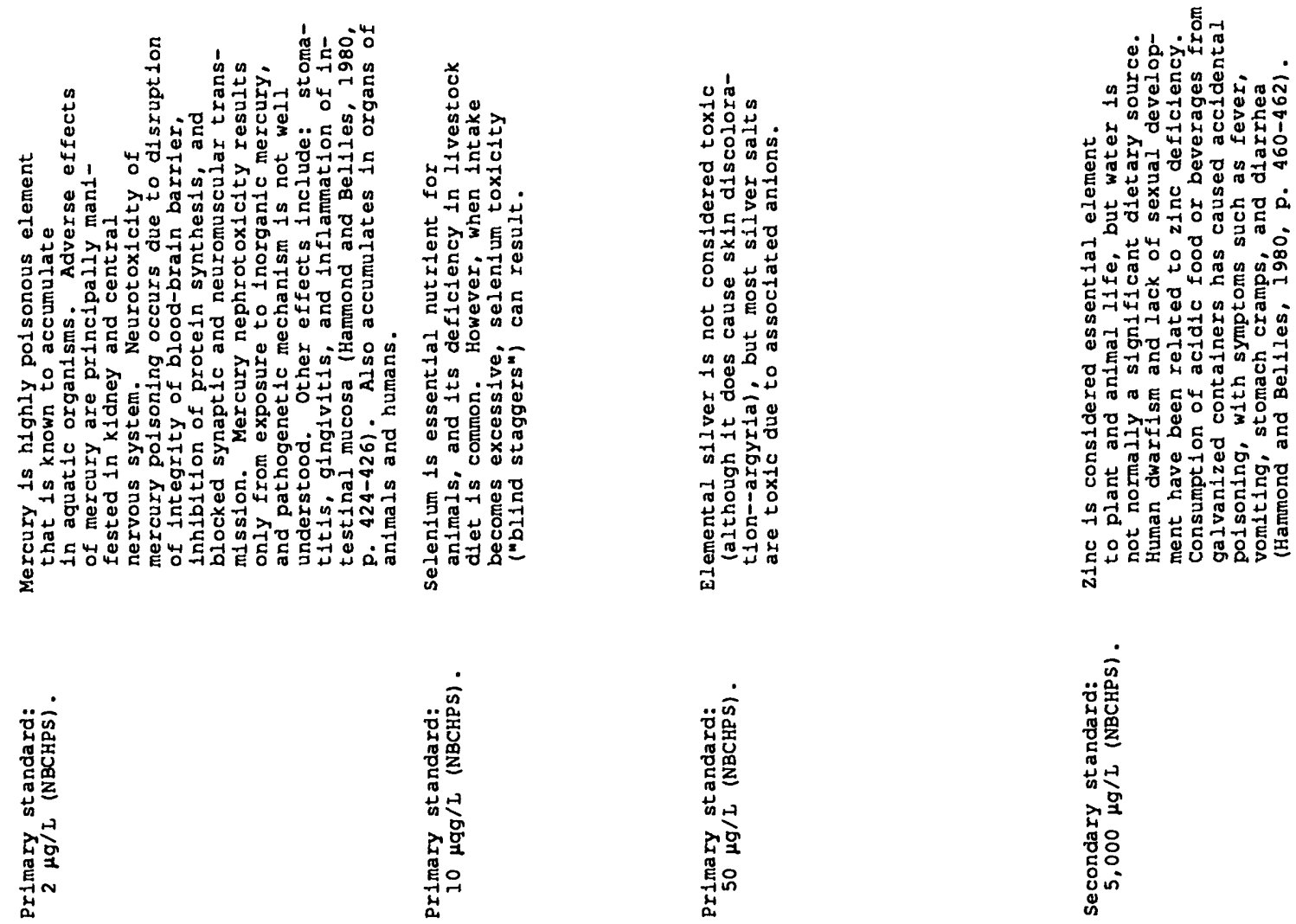

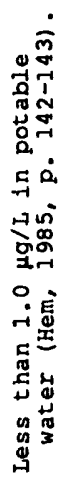
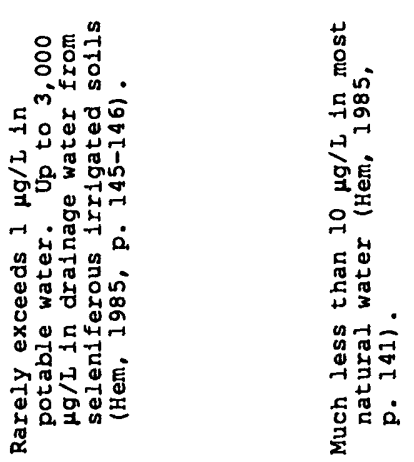

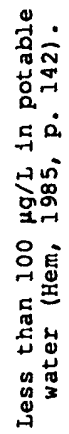
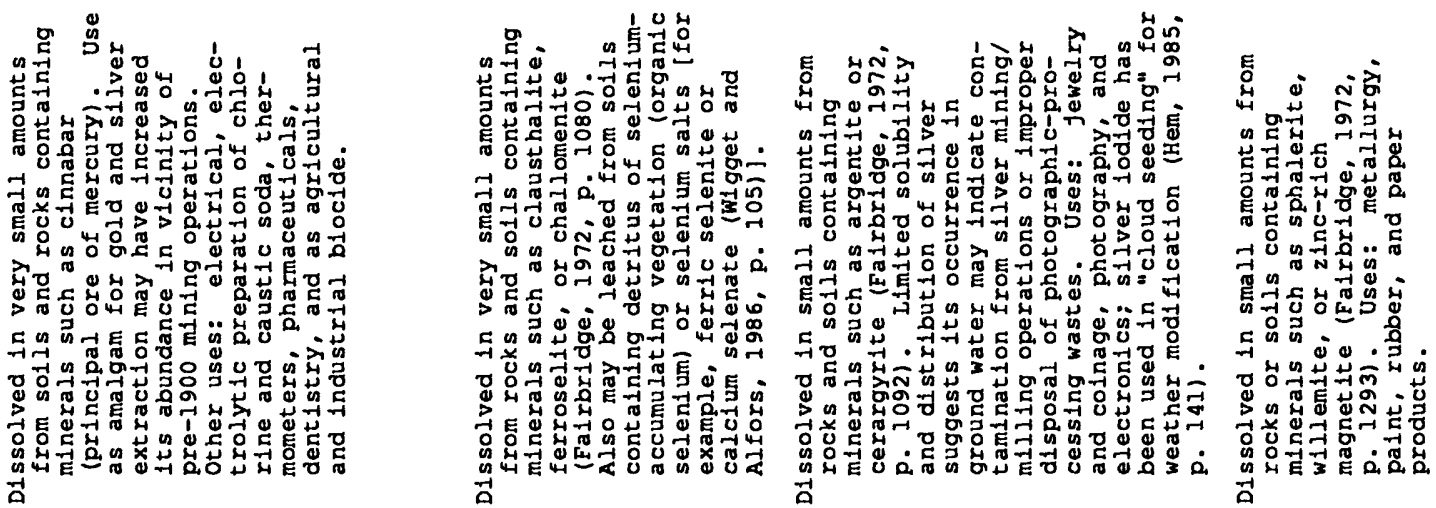

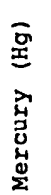

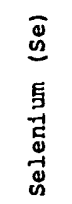

5
5
5
5
-1
2

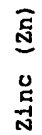




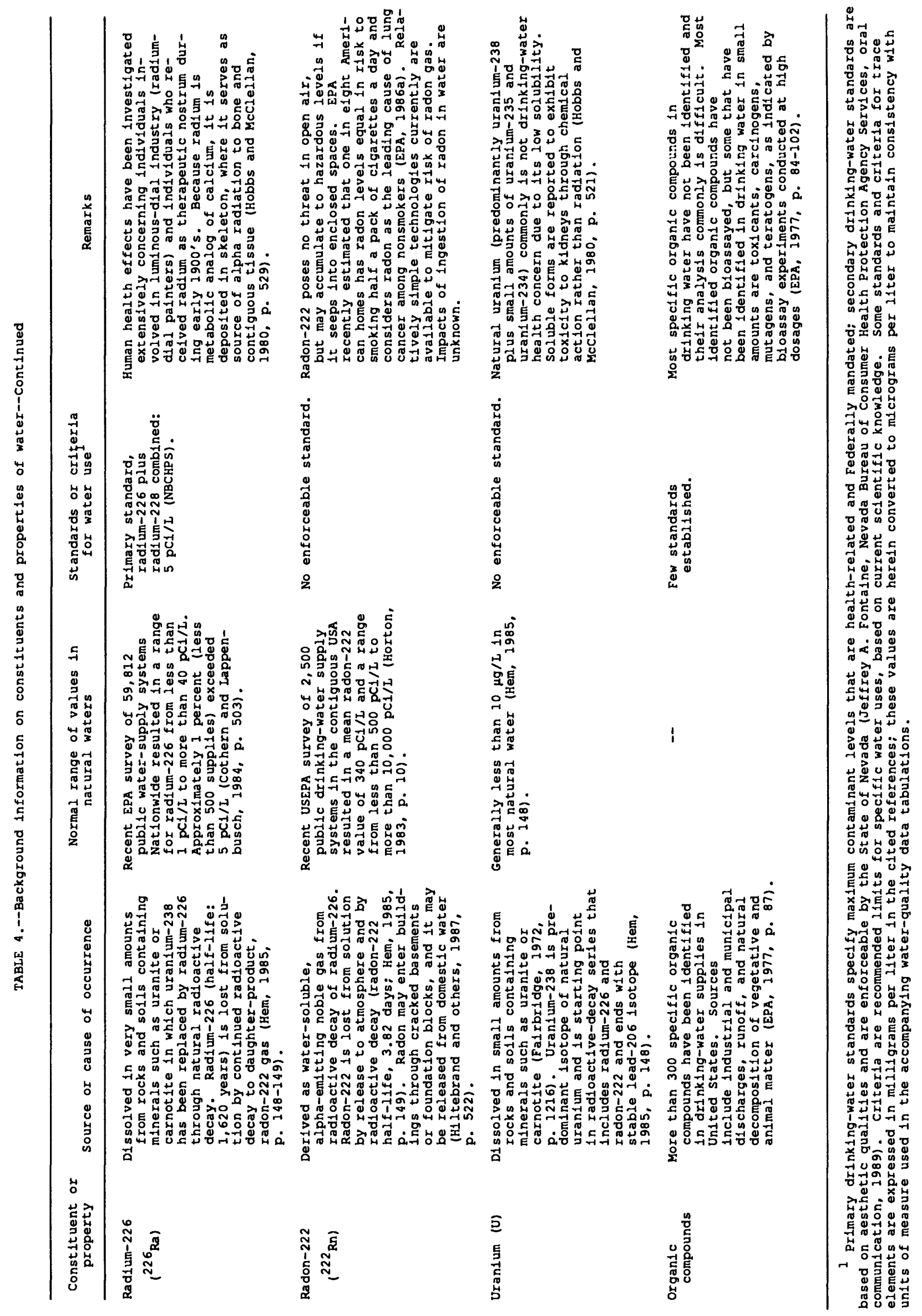


Primary drinking-water standards were exceeded for arsenic ( $50 \mu \mathrm{g} / \mathrm{L}$ ) at one observation well, for fluoride $(4.0 \mathrm{mg} / \mathrm{L})$ at one observation well, and for nitrate-nitrogen ( $10 \mathrm{mg} / \mathrm{L}$ ) at one domestic well. Secondary drinking-water standards were exceeded as follows: $\mathrm{pH}$ (6.5-8.5 units) at one public-supply well and one domestic well, sulfate (250 mg/L) at one observation well, dissolved solids $(500 \mathrm{mg} / \mathrm{L})$ at two observation wells, iron $(600 \mu \mathrm{g} / \mathrm{L})$ at one observation well, and manganese $(50 \mu \mathrm{g} / \mathrm{L})$ at three observation wells and one public-supply well. Seven wells (two domestic and five observation) had overall concentrations of unidentified organic compounds greater than $10 \mu \mathrm{g} / \mathrm{L}$ relative to the internal standard compound perdeuteronapthalene. Locations where primary drinking-water regulations and secondary drinking-water regulations have been exceeded are illustrated in figures 3 and 4, respectively, and locations where organic compounds were detected are shown in figure 5.

Regarding water-quality data listed for site 4 (tables 5-8), about 1,700 gallons of hydrochloric acid was added to the well during the first week of February 1986 to improve its yield. Between that time and the collection of the first water-quality sample from site 4 (on February 28 , 1986), an estimated 2 million gallons of water was withdrawn from the well (Car1 Bricker, Gardnerville Ranchos General Improvement District, oral communication, 1986). 


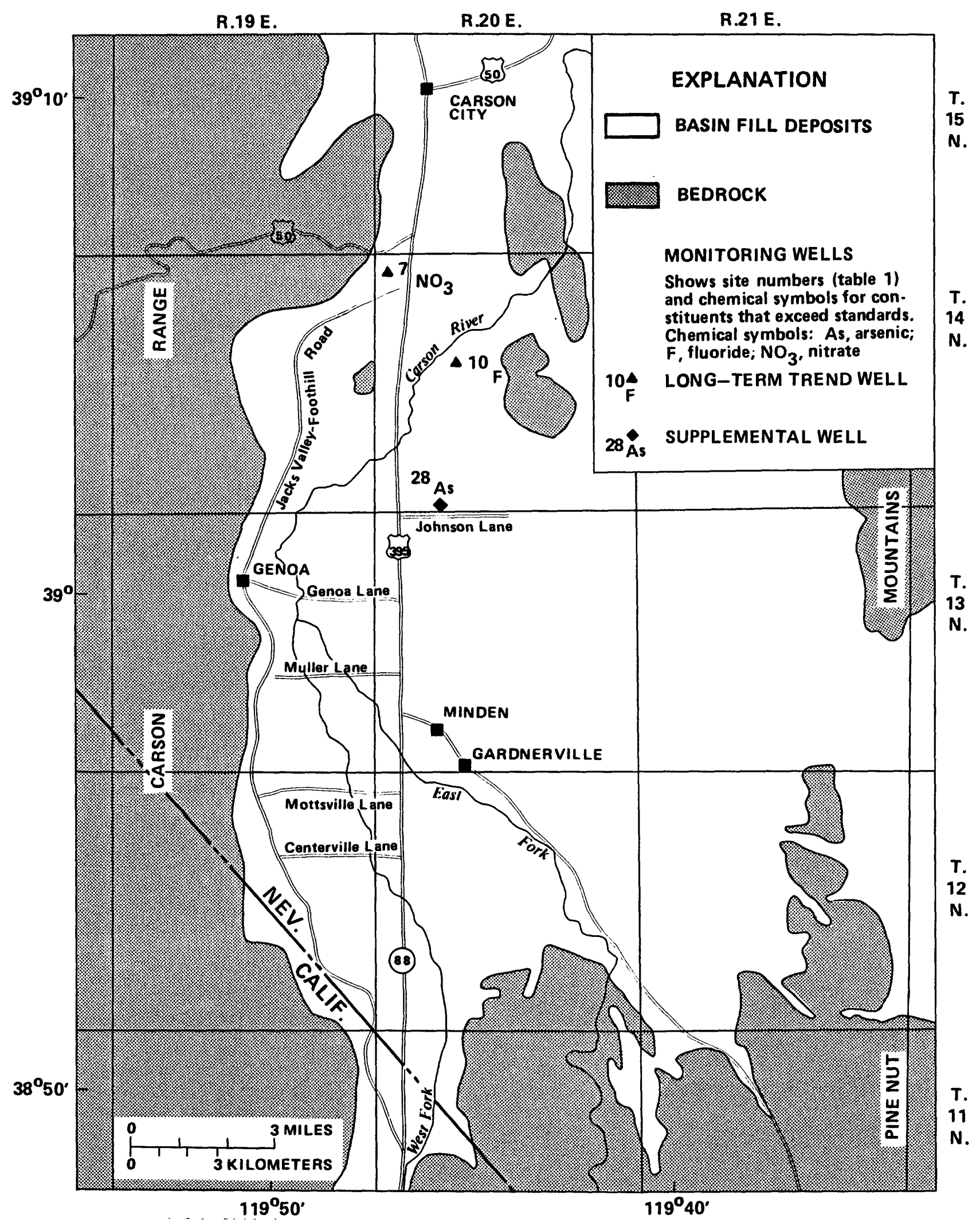

FIGURE 3.--Monitoring wells in Carson Valley where primary drinking-water standards were exceeded for indicated constituent. Note: no standards were exceeded in Topaz Lake area monitoring wells. 
R.19 E.

R.20 E.

R.21 E.

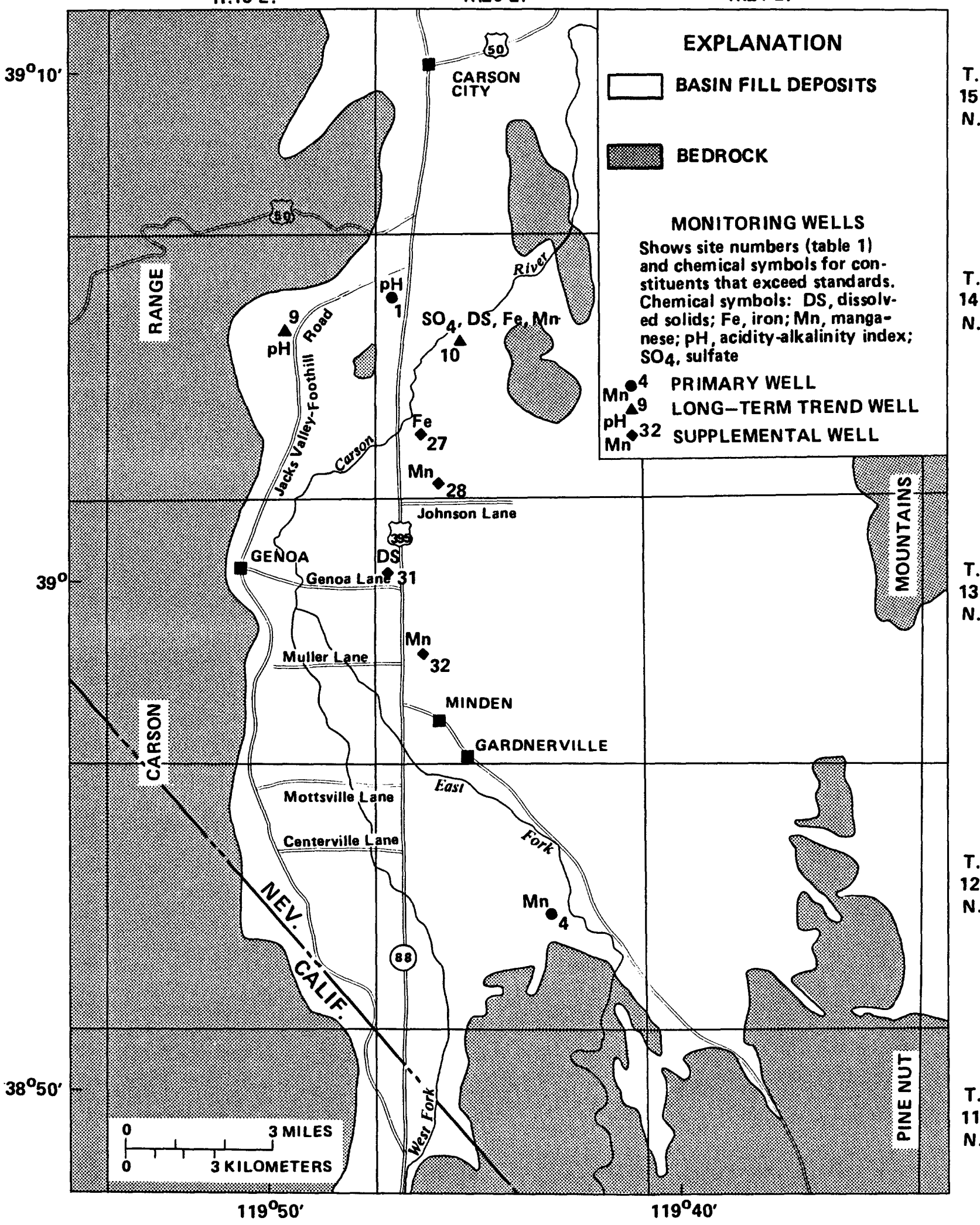

T.

N.

T.

14

N.

$T$.

$T$.

11

$N$.

FIGURE 4.--Monitoring wells in Carson Valley where secondary drinking-water standards were exceeded for indicated constituent. Note: no standards were exceeded in Topaz Lake area monitoring wells. 


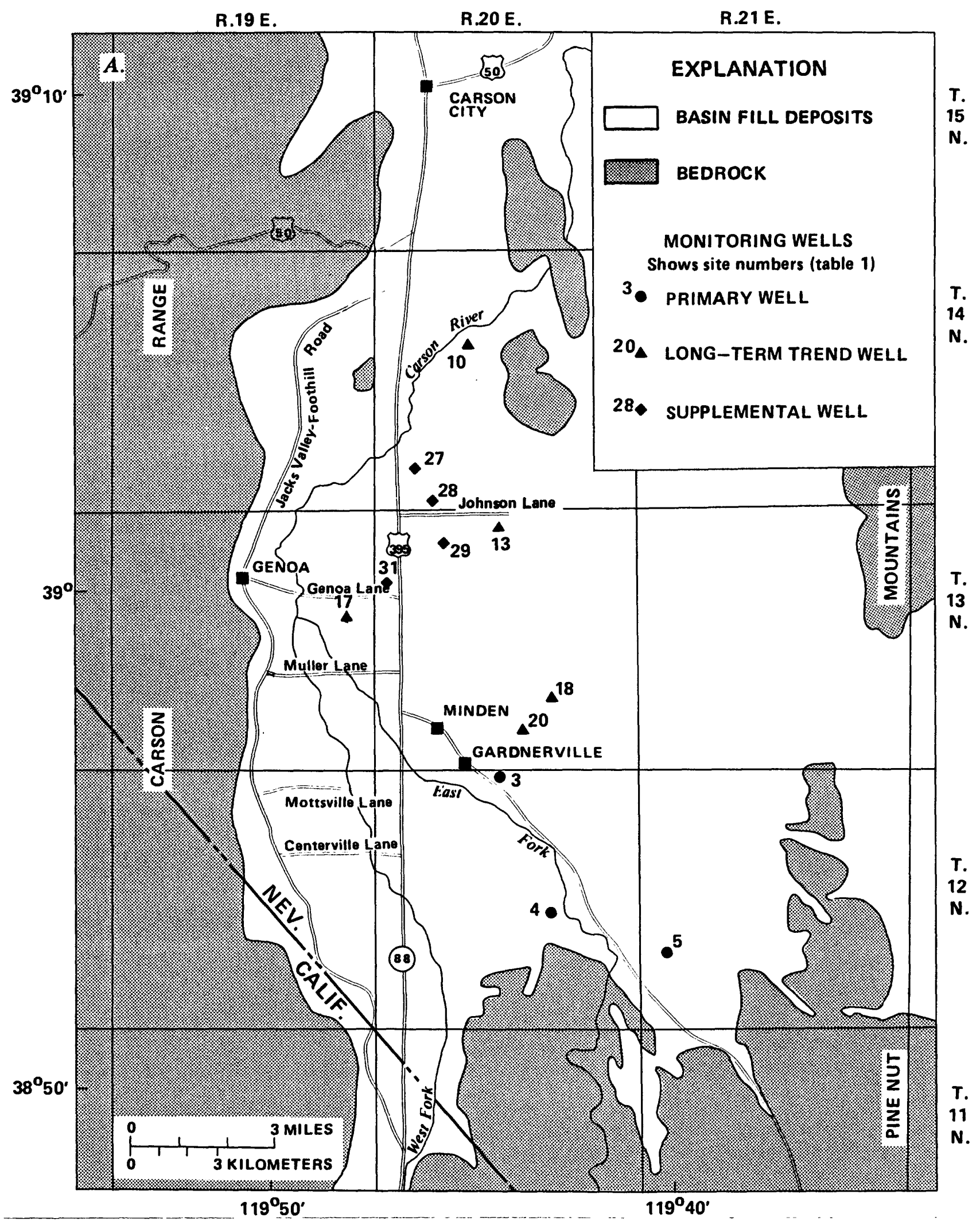

FIGURE 5.-Monitoring wells where organic compounds were detected (but not individually identified) by gas-chromatography and flame-ionization analysis in $(A)$ Carson Valley and $(B)$ Topaz Lake area. 


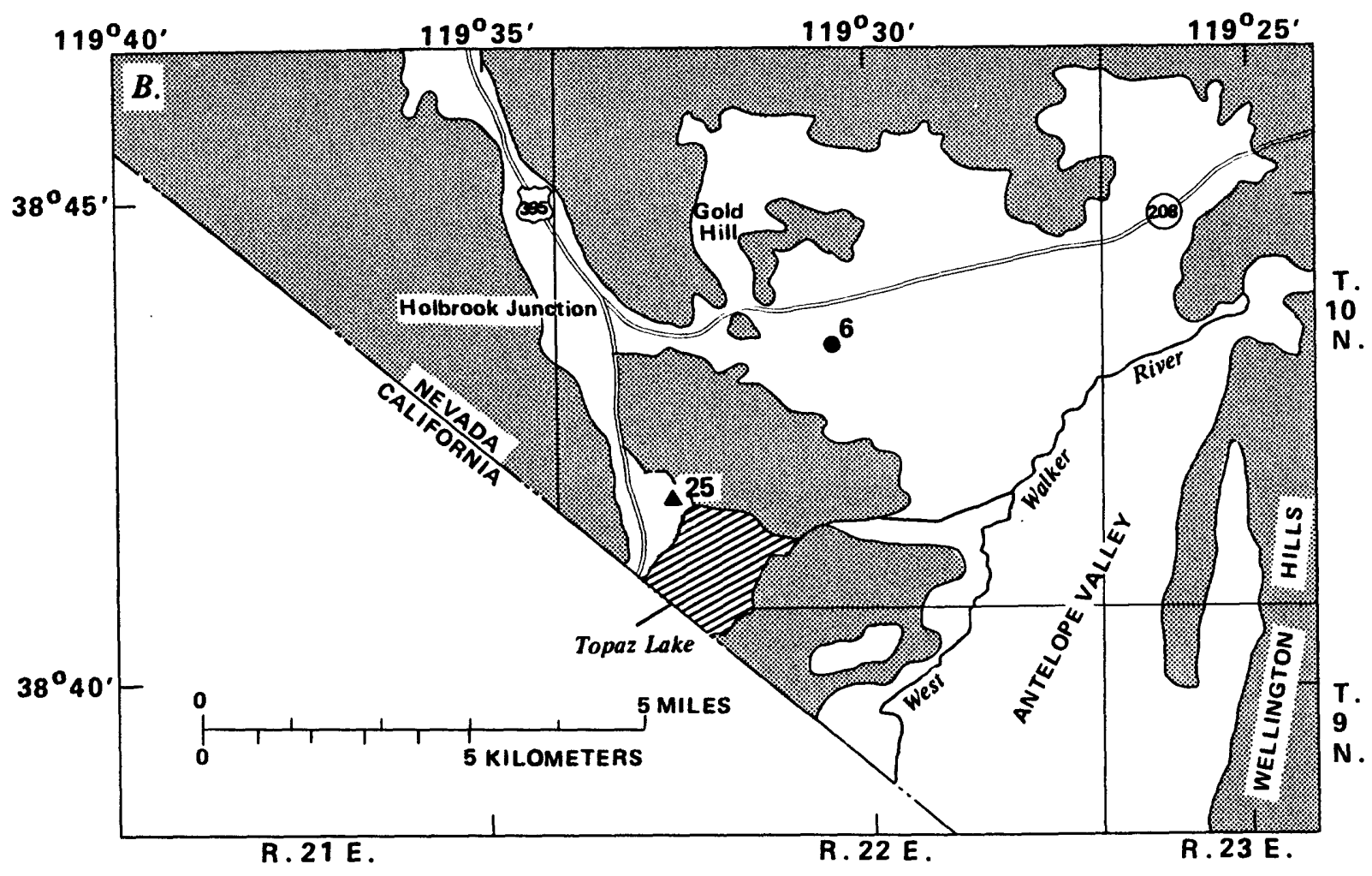

FIGURE 5.--Continued.

page 25 follows 


\section{DATA}

\section{TABULATIONS}


TABLE 5.--Data on physical properties and major chemical constituents

[Abbreviations: DEG C, degrees Celsius; IT, incremental titration; MG/L, milligrams per liter; NrU, nephelometric turbidity units; UG/L, micrograms per liter; US/CM, microsiemens per centimeter at $25^{\circ} \mathrm{C}$; <, less than; --, data not avallable]

\begin{tabular}{|c|c|c|c|c|c|c|c|c|}
\hline $\begin{array}{c}\text { SITE } \\
\text { NUMBER } \\
\text { (figure 2) }\end{array}$ & DATE & TIME & $\begin{array}{l}\text { FIELD } \\
\text { SPE- } \\
\text { CIFIC } \\
\text { CON- } \\
\text { DUCT- } \\
\text { ANCE } \\
\text { (US/CM) }\end{array}$ & $\begin{array}{c}\text { FIELD } \\
\text { phl } \\
\text { (STAND- } \\
\text { ARD } \\
\text { UNITS) }\end{array}$ & $\begin{array}{c}\text { WATEK } \\
\text { TEMPER- } \\
\text { ATURE } \\
\text { (DEG C) }\end{array}$ & $\begin{array}{c}\text { TOTAL } \\
\text { HARD- } \\
\text { NESS } \\
(\mathrm{MG} / \mathrm{L} \\
\mathrm{AS} \\
\left.\mathrm{CACO}_{3}\right)\end{array}$ & $\begin{array}{l}\text { HARD- } \\
\text { NESS, } \\
\text { NONCAR- } \\
\text { BONATE } \\
(\mathrm{MG} / \mathrm{L} \\
\mathrm{AS} \\
\left.\mathrm{CACO}_{3}\right)\end{array}$ & $\begin{array}{l}\text { CALCIUM, } \\
\text { DIS- } \\
\text { SOLVED } \\
\text { (MG/L } \\
\text { AS CA) }\end{array}$ \\
\hline 1 & $\begin{array}{l}12-19-85 \\
01-31-86 \\
03-17-86 \\
05-09-86 \\
08-06-86 \\
09-04-86\end{array}$ & $\begin{array}{l}1515 \\
1600 \\
1550 \\
0930 \\
1415 \\
1505\end{array}$ & $\begin{array}{l}290 \\
310 \\
295 \\
215 \\
280 \\
250\end{array}$ & $\begin{array}{l}8.5 \\
8.6 \\
8.7 \\
9.1 \\
9.1 \\
9.0\end{array}$ & $\begin{array}{l}18.5 \\
18.5 \\
18.5 \\
18.5 \\
19.5 \\
19.5\end{array}$ & $\begin{array}{l}38 \\
45 \\
51 \\
21 \\
19 \\
21\end{array}$ & $\begin{array}{l}0 \\
0 \\
0 \\
0 \\
0 \\
0\end{array}$ & $\begin{array}{l}13 \\
15 \\
17 \\
7.9 \\
7.1 \\
7.8\end{array}$ \\
\hline 2 & $\begin{array}{l}11-18-85 \\
01-27-86 \\
03-17-86 \\
05-14-86\end{array}$ & $\begin{array}{l}1510 \\
1440 \\
1150 \\
1440\end{array}$ & $\begin{array}{l}210 \\
195 \\
195 \\
195\end{array}$ & $\begin{array}{l}8.2 \\
8.0 \\
8.5 \\
8.4\end{array}$ & $\begin{array}{l}13.0 \\
13.0 \\
13.0 \\
13.5\end{array}$ & $\begin{array}{l}71 \\
75 \\
71 \\
65\end{array}$ & $\begin{array}{l}0 \\
0 \\
0 \\
0\end{array}$ & $\begin{array}{l}22 \\
23 \\
22 \\
20\end{array}$ \\
\hline 3 & $\begin{array}{l}11-18-85 \\
01-28-86 \\
03-17-86 \\
05-16-86 \\
07-31-86 \\
09-04-86\end{array}$ & $\begin{array}{l}1315 \\
1415 \\
1400 \\
1040 \\
1220 \\
1050\end{array}$ & $\begin{array}{l}435 \\
385 \\
440 \\
410 \\
430 \\
425\end{array}$ & $\begin{array}{l}7.0 \\
7.1 \\
7.3 \\
7.1 \\
7.2 \\
7.2\end{array}$ & $\begin{array}{l}12.0 \\
12.0 \\
12.0 \\
12.5 \\
12.0 \\
12.0\end{array}$ & $\begin{array}{l}170 \\
170 \\
180 \\
160 \\
210 \\
160\end{array}$ & $\begin{array}{l}0 \\
1 \\
9 \\
0 \\
0 \\
1\end{array}$ & $\begin{array}{l}47 \\
45 \\
47 \\
44 \\
56 \\
44\end{array}$ \\
\hline 4 & $\begin{array}{l}02-28-86 \\
05-15-86 \\
07-30-86 \\
09-03-86\end{array}$ & $\begin{array}{l}0950 \\
1610 \\
1300 \\
1430\end{array}$ & $\begin{array}{l}400 \\
360 \\
205 \\
220\end{array}$ & $\begin{array}{l}6.8 \\
6.8 \\
7.1 \\
7.3\end{array}$ & $\begin{array}{l}12.0 \\
12.5 \\
12.0 \\
12.0\end{array}$ & $\begin{array}{r}150 \\
120 \\
82 \\
83\end{array}$ & $\begin{array}{r}82 \\
61 \\
8 \\
5\end{array}$ & $\begin{array}{l}40 \\
32 \\
22 \\
22\end{array}$ \\
\hline 5 & $\begin{array}{l}11-19-85 \\
01-29-86 \\
03-19-86 \\
05-15-86 \\
07-30-86 \\
09-03-86\end{array}$ & $\begin{array}{l}1145 \\
1115 \\
1400 \\
1040 \\
1000 \\
1245\end{array}$ & $\begin{array}{l}400 \\
395 \\
385 \\
400 \\
410 \\
395\end{array}$ & $\begin{array}{l}7.7 \\
7.7 \\
8.0 \\
8.0 \\
8.1 \\
8.0\end{array}$ & $\begin{array}{l}21.5 \\
21.0 \\
21.5 \\
22.0 \\
21.0 \\
21.5\end{array}$ & $\begin{array}{l}86 \\
90 \\
87 \\
81 \\
86 \\
84\end{array}$ & $\begin{array}{l}0 \\
0 \\
0 \\
0 \\
0 \\
0\end{array}$ & $\begin{array}{l}28 \\
29 \\
28 \\
26 \\
28 \\
27\end{array}$ \\
\hline 6 & $\begin{array}{l}11-15-85 \\
01-27-86 \\
03-19-86 \\
05-12-86 \\
08-04-86 \\
09-0.3-86\end{array}$ & $\begin{array}{l}1035 \\
0930 \\
1100 \\
1040 \\
1010 \\
1015\end{array}$ & $\begin{array}{l}540 \\
505 \\
490 \\
495 \\
490 \\
495\end{array}$ & $\begin{array}{l}7.4 \\
7.4 \\
7.5 \\
7.7 \\
7.7 \\
7.6\end{array}$ & $\begin{array}{l}15.0 \\
14.5 \\
15.5 \\
15.5 \\
15.0 \\
15.5\end{array}$ & $\begin{array}{l}240 \\
240 \\
220 \\
210 \\
220 \\
230\end{array}$ & $\begin{array}{l}47 \\
47 \\
50 \\
44 \\
48 \\
48\end{array}$ & $\begin{array}{l}70 \\
70 \\
65 \\
64 \\
65 \\
67\end{array}$ \\
\hline
\end{tabular}


TABLE 5.--Data on physical properties and major chemical constituents - - Continued

\begin{tabular}{|c|c|c|c|c|c|c|c|}
\hline $\begin{array}{c}\text { SITE } \\
\text { NUMBER } \\
\text { (f1gure 2) }\end{array}$ & DATE & $\begin{array}{l}\text { MAGNE- } \\
\text { SIUM, } \\
\text { DIS- } \\
\text { SOLVED } \\
\text { (MG/L } \\
\text { AS MG) }\end{array}$ & $\begin{array}{l}\text { SODIUM, } \\
\text { DIS- } \\
\text { SOLVED } \\
\text { (MG/L } \\
\text { AS NA) }\end{array}$ & $\begin{array}{l}\text { POTAS- } \\
\text { SIUM, } \\
\text { DIS- } \\
\text { SOLVED } \\
\text { (MG/L } \\
\text { AS K) }\end{array}$ & $\begin{array}{l}\text { BICAR- } \\
\text { BONATE, } \\
\text { IT-FIELD } \\
(\mathrm{MG} / \mathrm{L} \\
\left.\text { AS } \mathrm{HCO}_{3}\right)\end{array}$ & $\begin{array}{c}\text { CAR- } \\
\text { BONATE, } \\
\text { IT-FIELD } \\
(\mathrm{MG} / \mathrm{L} \\
\left.\mathrm{AS} \mathrm{CO}_{3}\right)\end{array}$ & $\begin{array}{l}\text { ALKA- } \\
\text { LINITY, } \\
\text { CARBON- } \\
\text { ATE, } \\
\text { IT-FIELD } \\
(\mathrm{MG/L} \mathrm{AS} \\
\left.\mathrm{CACO}_{3}\right)\end{array}$ \\
\hline 1 & $\begin{array}{l}12-19-85 \\
01-31-86 \\
03-17-86 \\
05-09-86 \\
08-06-86 \\
09-04-86\end{array}$ & $\begin{array}{l}1.4 \\
1.8 \\
2.0 \\
.4 \\
.2 \\
.3\end{array}$ & $\begin{array}{l}47 \\
44 \\
45 \\
46 \\
47 \\
48\end{array}$ & $\begin{array}{l}0.6 \\
.9 \\
.9 \\
.6 \\
.6 \\
.7\end{array}$ & $\begin{array}{r}123 \\
123 \\
124 \\
101 \\
85 \\
95\end{array}$ & $\begin{array}{r}2 \\
4 \\
5 \\
9 \\
10 \\
7\end{array}$ & $\begin{array}{r}102 \\
107 \\
110 \\
86 \\
87 \\
90\end{array}$ \\
\hline 2 & $\begin{array}{l}11-18-85 \\
01-27-86 \\
03-17-86 \\
05-14-86\end{array}$ & $\begin{array}{l}3.9 \\
4.3 \\
3.8 \\
3.6\end{array}$ & $\begin{array}{l}13 \\
13 \\
13 \\
13\end{array}$ & $\begin{array}{l}2.3 \\
2.3 \\
2.3 \\
2.3\end{array}$ & $\begin{array}{r}95 \\
106 \\
87 \\
92\end{array}$ & $\begin{array}{l}0 \\
0 \\
3 \\
0\end{array}$ & $\begin{array}{l}78 \\
87 \\
76 \\
76\end{array}$ \\
\hline 3 & $\begin{array}{l}11-18-85 \\
01-28-86 \\
03-17-86 \\
05-16-86 \\
07-31-86 \\
09-04-86\end{array}$ & $\begin{array}{l}13 \\
13 \\
14 \\
13 \\
16 \\
13\end{array}$ & $\begin{array}{l}21 \\
21 \\
22 \\
20 \\
25 \\
22\end{array}$ & $\begin{array}{l}3.0 \\
3.0 \\
3.2 \\
3.0 \\
3.2 \\
2.8\end{array}$ & $\begin{array}{l}210 \\
201 \\
203 \\
206 \\
255 \\
199\end{array}$ & $\begin{array}{l}0 \\
0 \\
0 \\
0 \\
0 \\
0\end{array}$ & $\begin{array}{l}180 \\
165 \\
166 \\
169 \\
209 \\
163\end{array}$ \\
\hline 4 & $\begin{array}{l}02-28-86 \\
05-15-86 \\
07-30-86 \\
09-03-86\end{array}$ & $\begin{array}{l}11 \\
9.8 \\
6.6 \\
6.7\end{array}$ & $\begin{array}{l}15 \\
17 \\
14 \\
14\end{array}$ & $\begin{array}{l}2.3 \\
2.3 \\
1.9 \\
1.8\end{array}$ & $\begin{array}{l}77 \\
72 \\
90 \\
95\end{array}$ & $\begin{array}{l}0 \\
0 \\
0 \\
0\end{array}$ & $\begin{array}{l}63 \\
59 \\
74 \\
78\end{array}$ \\
\hline 5 & $\begin{array}{l}11-19-85 \\
01-29-86 \\
03-19-86 \\
05-15-86 \\
07-30-86 \\
09-03-86\end{array}$ & $\begin{array}{l}4.0 \\
4.2 \\
4.1 \\
3.9 \\
4.0 \\
4.0\end{array}$ & $\begin{array}{l}47 \\
48 \\
47 \\
45 \\
48 \\
47\end{array}$ & $\begin{array}{l}2.4 \\
2.4 \\
2.5 \\
2.4 \\
2.5 \\
2.3\end{array}$ & $\begin{array}{l}150 \\
156 \\
143 \\
142 \\
150 \\
146\end{array}$ & $\begin{array}{l}0 \\
0 \\
0 \\
0 \\
0 \\
0\end{array}$ & $\begin{array}{l}120 \\
128 \\
117 \\
116 \\
123 \\
120\end{array}$ \\
\hline 6 & $\begin{array}{l}11-15-85 \\
01-27-86 \\
03-19-86 \\
05-12-86 \\
08-04-86 \\
09-03-86\end{array}$ & $\begin{array}{l}15 \\
16 \\
14 \\
13 \\
14 \\
15\end{array}$ & $\begin{array}{l}19 \\
19 \\
19 \\
19 \\
20 \\
20\end{array}$ & $\begin{array}{l}1.2 \\
1.1 \\
1.1 \\
1.1 \\
1.0 \\
1.1\end{array}$ & $\begin{array}{l}230 \\
237 \\
207 \\
206 \\
210 \\
221\end{array}$ & $\begin{array}{l}0 \\
0 \\
0 \\
0 \\
0 \\
0\end{array}$ & $\begin{array}{l}190 \\
194 \\
170 \\
169 \\
172 \\
181\end{array}$ \\
\hline
\end{tabular}


TABLE 5.--Data on physical properties and major chemical constituents--Continued

\begin{tabular}{|c|c|c|c|c|c|c|c|c|}
\hline $\begin{array}{c}\text { SITE } \\
\text { NUMBER } \\
\text { (figure 2) }\end{array}$ & DATE & $\begin{array}{l}\text { SULFATE, } \\
\text { DIS- } \\
\text { SOLVED } \\
(\mathrm{MG} / \mathrm{L} \\
\left.\mathrm{AS} \mathrm{SO}_{4}\right)\end{array}$ & $\begin{array}{l}\text { CHLO- } \\
\text { RIDE, } \\
\text { DIS- } \\
\text { SOLVED } \\
\text { (MG/L } \\
\text { AS CL) }\end{array}$ & $\begin{array}{l}\text { FLUO- } \\
\text { RIDE, } \\
\text { DIS- } \\
\text { SOLVED } \\
\text { (MG/L } \\
\text { AS F) }\end{array}$ & $\begin{array}{l}\text { SILICA, } \\
\text { DIS- } \\
\text { SOLVED } \\
\text { (MG/L } \\
\text { AS } \\
\mathrm{SIO}_{2} \text { ) }\end{array}$ & $\begin{array}{l}\text { SOLIDS, } \\
\text { RESIDUE } \\
\text { AT 180 } \\
\text { DEG C, } \\
\text { DIS- } \\
\text { SOLVED } \\
(\mathrm{MG} / \mathrm{L})\end{array}$ & $\begin{array}{l}\text { SOLIDS, } \\
\text { SUM OF } \\
\text { CONSTI- } \\
\text { TUENTS, } \\
\text { DIS- } \\
\text { SOLVED } \\
\text { (MG/L) }\end{array}$ & $\begin{array}{c}\text { TUR- } \\
\text { BID- } \\
\text { ITY } \\
\text { (NTU) }\end{array}$ \\
\hline 1 & $\begin{array}{l}12-19-85 \\
01-31-86 \\
03-17-86 \\
05-09-86 \\
08-06-86 \\
09-04-86\end{array}$ & $\begin{array}{l}15 \\
15 \\
16 \\
16 \\
16 \\
16\end{array}$ & $\begin{array}{l}10 \\
13 \\
12 \\
12 \\
10 \\
10\end{array}$ & $\begin{array}{r}0.4 \\
.4 \\
.4 \\
.3 \\
.4 \\
.4\end{array}$ & $\begin{array}{l}23 \\
24 \\
24 \\
19 \\
18 \\
19\end{array}$ & $\begin{array}{l}166 \\
179 \\
181 \\
157 \\
161 \\
161\end{array}$ & $\begin{array}{l}180 \\
180 \\
190 \\
170 \\
160 \\
160\end{array}$ & $\begin{array}{r}1.7 \\
.6 \\
.8 \\
.8 \\
1.0 \\
-\end{array}$ \\
\hline 2 & $\begin{array}{l}11-18-85 \\
01-27-86 \\
03-17-86 \\
05-14-86\end{array}$ & $\begin{array}{l}19 \\
11 \\
14 \\
16\end{array}$ & $\begin{array}{l}4.7 \\
4.6 \\
4.6 \\
4.5\end{array}$ & $\begin{array}{r}<.1 \\
<.1 \\
.1 \\
.1\end{array}$ & $\begin{array}{l}30 \\
30 \\
29 \\
30\end{array}$ & $\begin{array}{l}127 \\
137 \\
130 \\
129\end{array}$ & $\begin{array}{l}140 \\
140 \\
140 \\
140\end{array}$ & $\begin{array}{r}.7 \\
.8 \\
.6 \\
1.0\end{array}$ \\
\hline 3 & $\begin{array}{l}11-18-85 \\
01-28-86 \\
03-17-86 \\
05-16-86 \\
07-31-86 \\
09-04-86\end{array}$ & $\begin{array}{l}31 \\
22 \\
29 \\
32 \\
33 \\
33\end{array}$ & $\begin{array}{l}11 \\
9.9 \\
10 \\
13 \\
8.3 \\
9.3\end{array}$ & $\begin{array}{l}<.1 \\
<.1 \\
<.1 \\
.1 \\
<.1 \\
<.1\end{array}$ & $\begin{array}{l}31 \\
31 \\
31 \\
30 \\
31 \\
31\end{array}$ & $\begin{array}{l}250 \\
242 \\
258 \\
254 \\
306 \\
240\end{array}$ & $\begin{array}{l}260 \\
250 \\
260 \\
260 \\
300 \\
250\end{array}$ & $\begin{array}{r}.5 \\
.3 \\
.4 \\
1.0 \\
.5\end{array}$ \\
\hline 4 & $\begin{array}{l}02-28-86 \\
05-15-86 \\
07-30-86 \\
09-03-86\end{array}$ & $\begin{array}{l}18 \\
34 \\
24 \\
24\end{array}$ & $\begin{array}{l}71 \\
46 \\
6.2 \\
6.3\end{array}$ & $\begin{array}{r}.2 \\
.3 \\
<.1 \\
.1\end{array}$ & $\begin{array}{l}32 \\
43 \\
34 \\
33\end{array}$ & $\begin{array}{l}264 \\
255 \\
165 \\
151\end{array}$ & $\begin{array}{l}230 \\
220 \\
150 \\
150\end{array}$ & $\begin{array}{l}1.5 \\
3.0 \\
1.0 \\
-\end{array}$ \\
\hline 5 & $\begin{array}{l}11-19-85 \\
01-29-86 \\
03-19-86 \\
05-15-86 \\
07-30-86 \\
09-03-86\end{array}$ & $\begin{array}{l}32 \\
20 \\
23 \\
32 \\
35 \\
35\end{array}$ & $\begin{array}{l}15 \\
13 \\
14 \\
14 \\
15 \\
13\end{array}$ & $\begin{array}{r}<.1 \\
.1 \\
.2 \\
.1 \\
<.1 \\
.1\end{array}$ & $\begin{array}{l}31 \\
31 \\
31 \\
31 \\
30 \\
30\end{array}$ & $\begin{array}{l}245 \\
252 \\
236 \\
243 \\
262 \\
240\end{array}$ & $\begin{array}{l}230 \\
240 \\
220 \\
220 \\
240 \\
230\end{array}$ & $\begin{array}{r}.5 \\
4.4 \\
.3 \\
1.0 \\
.6\end{array}$ \\
\hline 6 & $\begin{array}{l}11-15-85 \\
01-27-86 \\
03-19-86 \\
05-12-86 \\
08-04-86 \\
09-03-86\end{array}$ & $\begin{array}{l}79 \\
80 \\
77 \\
80 \\
75 \\
88\end{array}$ & $\begin{array}{l}3.4 \\
3.6 \\
3.1 \\
3.3 \\
3.2 \\
4.0\end{array}$ & $\begin{array}{l}.1 \\
.1 \\
.1 \\
.1 \\
.1 \\
.1\end{array}$ & $\begin{array}{l}25 \\
25 \\
25 \\
25 \\
25 \\
25\end{array}$ & $\begin{array}{l}316 \\
327 \\
303 \\
308 \\
323 \\
300\end{array}$ & $\begin{array}{l}330 \\
330 \\
310 \\
310 \\
310 \\
330\end{array}$ & $\begin{array}{l}.8 \\
.3 \\
.5 \\
.5 \\
.6 \\
-.\end{array}$ \\
\hline
\end{tabular}


TABLE 5.-Data on physical properties and major chemical constituents-.Continued

\begin{tabular}{|c|c|c|c|c|c|c|c|c|}
\hline $\begin{array}{c}\text { SITE } \\
\text { NUMBER } \\
\text { (f1gure 2) }\end{array}$ & DATE & TIME & $\begin{array}{l}\text { FIELD } \\
\text { SPE- } \\
\text { CIFIC } \\
\text { CON- } \\
\text { DUCT- } \\
\text { ANCE } \\
\text { (US/CM) }\end{array}$ & $\begin{array}{c}\text { FIELD } \\
\text { pH } \\
\text { (STAND- } \\
\text { ARD } \\
\text { UNITS) }\end{array}$ & $\begin{array}{c}\text { WATER } \\
\text { TEMPER- } \\
\text { ATURE } \\
\text { (DEG C) }\end{array}$ & $\begin{array}{c}\text { TOTAL } \\
\text { HARD- } \\
\text { NESS } \\
(\mathrm{MG} / \mathrm{L} \\
\text { AS } \\
\left.\mathrm{CACO}_{3}\right)\end{array}$ & $\begin{array}{l}\text { HARD- } \\
\text { NESS, } \\
\text { NONCAR- } \\
\text { BONATE } \\
(\mathrm{MG} / \mathrm{L} \\
\text { AS } \\
\left.\mathrm{CACO}_{3}\right)\end{array}$ & $\begin{array}{l}\text { CALCIUM, } \\
\text { DIS- } \\
\text { SOLVED } \\
\text { (MG/L } \\
\text { AS CA) }\end{array}$ \\
\hline 7 & $\begin{array}{l}12-03-85 \\
02-27-86 \\
05-09-86 \\
08-11-86\end{array}$ & $\begin{array}{l}1415 \\
0945 \\
1240 \\
1010\end{array}$ & $\begin{array}{l}735 \\
685 \\
810 \\
750\end{array}$ & $\begin{array}{l}6.9 \\
7.2 \\
7.1 \\
7.3\end{array}$ & $\begin{array}{l}13.0 \\
12.5 \\
13.0 \\
12.5\end{array}$ & $\begin{array}{l}230 \\
240 \\
250 \\
240\end{array}$ & $\begin{array}{l}55 \\
77 \\
81 \\
76\end{array}$ & $\begin{array}{l}75 \\
77 \\
80 \\
77\end{array}$ \\
\hline 8 & $\begin{array}{l}12-03-85 \\
02-26-86 \\
05-09-86 \\
08-19-86\end{array}$ & $\begin{array}{l}1215 \\
1505 \\
1450 \\
1240\end{array}$ & $\begin{array}{l}255 \\
245 \\
265 \\
255\end{array}$ & $\begin{array}{l}7.8 \\
8.1 \\
8.2 \\
8.1\end{array}$ & $\begin{array}{l}12.5 \\
13.0 \\
13.5 \\
14.5\end{array}$ & $\begin{array}{l}50 \\
51 \\
50 \\
50\end{array}$ & $\begin{array}{l}0 \\
0 \\
0 \\
0\end{array}$ & $\begin{array}{l}17 \\
17 \\
17 \\
17\end{array}$ \\
\hline 9 & $\begin{array}{l}11-22-85 \\
02-27-86 \\
05-09-86 \\
08-12-86\end{array}$ & $\begin{array}{l}0950 \\
1230 \\
1600 \\
1440\end{array}$ & $\begin{array}{l}120 \\
125 \\
125 \\
115\end{array}$ & $\begin{array}{l}8.6 \\
8.7 \\
8.8 \\
8.7\end{array}$ & $\begin{array}{l}13.5 \\
14.5 \\
15.0 \\
14.5\end{array}$ & $\begin{array}{l}33 \\
33 \\
33 \\
--\end{array}$ & $\begin{array}{l}0 \\
0 \\
0 \\
--\end{array}$ & $\begin{array}{l}13 \\
13 \\
13 \\
13\end{array}$ \\
\hline 10 & $\begin{array}{l}12-09-85 \\
02-10-86 \\
05-19-86 \\
08-13-86\end{array}$ & $\begin{array}{l}1210 \\
1200 \\
1150 \\
1020\end{array}$ & $\begin{array}{l}2850 \\
2800 \\
3000 \\
2950\end{array}$ & $\begin{array}{l}6.6 \\
6.8 \\
6.7 \\
6.8\end{array}$ & $\begin{array}{l}13.0 \\
13.0 \\
13.5 \\
13.0\end{array}$ & $\begin{array}{l}500 \\
500 \\
520 \\
570\end{array}$ & $\begin{array}{l}350 \\
360 \\
390 \\
440\end{array}$ & $\begin{array}{l}190 \\
190 \\
200 \\
220\end{array}$ \\
\hline 11 & $\begin{array}{l}11-20-85 \\
02-26-86 \\
05-20-86 \\
08-13-86\end{array}$ & $\begin{array}{l}1445 \\
1215 \\
1430 \\
1200\end{array}$ & $\begin{array}{l}480 \\
470 \\
470 \\
475\end{array}$ & $\begin{array}{l}7.7 \\
7.9 \\
8.0 \\
8.0\end{array}$ & $\begin{array}{l}15.0 \\
15.0 \\
15.0 \\
15.0\end{array}$ & $\begin{array}{r}99 \\
110 \\
100 \\
100\end{array}$ & $\begin{array}{l}0 \\
0 \\
0 \\
0\end{array}$ & $\begin{array}{l}34 \\
36 \\
34 \\
34\end{array}$ \\
\hline 12 & $\begin{array}{l}11-22-85 \\
02-12-86 \\
05-20-86 \\
08-13-86\end{array}$ & $\begin{array}{l}1245 \\
1400 \\
1230 \\
1400\end{array}$ & $\begin{array}{l}350 \\
355 \\
355 \\
350\end{array}$ & $\begin{array}{l}7.4 \\
7.8 \\
7.7 \\
7.8\end{array}$ & $\begin{array}{l}15.5 \\
15.5 \\
16.0 \\
15.5\end{array}$ & $\begin{array}{l}53 \\
54 \\
53 \\
54\end{array}$ & $\begin{array}{l}0 \\
0 \\
0 \\
0\end{array}$ & $\begin{array}{l}14 \\
14 \\
14 \\
14\end{array}$ \\
\hline 13 & $\begin{array}{l}11-22-85 \\
02-12-86 \\
05-20-86 \\
08-27-86\end{array}$ & $\begin{array}{l}1515 \\
1200 \\
1000 \\
1040\end{array}$ & $\begin{array}{l}360 \\
350 \\
370 \\
355\end{array}$ & $\begin{array}{l}7.8 \\
8.0 \\
8.0 \\
8.0\end{array}$ & $\begin{array}{l}20.5 \\
20.0 \\
21.0 \\
21.0\end{array}$ & $\begin{array}{l}35 \\
35 \\
36 \\
37\end{array}$ & $\begin{array}{l}0 \\
0 \\
0 \\
0\end{array}$ & $\begin{array}{l}9.2 \\
9.3 \\
9.5 \\
9.7\end{array}$ \\
\hline 14 & $\begin{array}{l}11-25-85 \\
02-06-86 \\
05-13-86 \\
08-18-86\end{array}$ & $\begin{array}{l}1335 \\
1030 \\
1200 \\
1345\end{array}$ & $\begin{array}{l}300 \\
290 \\
310 \\
305\end{array}$ & $\begin{array}{l}6.9 \\
7.3 \\
7.3 \\
7.2\end{array}$ & $\begin{array}{l}11.0 \\
11.0 \\
11.0 \\
11.0\end{array}$ & $\begin{array}{l}130 \\
120 \\
120 \\
130\end{array}$ & $\begin{array}{l}0 \\
0 \\
0 \\
0\end{array}$ & $\begin{array}{l}43 \\
39 \\
40 \\
41\end{array}$ \\
\hline
\end{tabular}


TABLE 5.--Data on physical properties and major chemical constituents--Continued

\begin{tabular}{|c|c|c|c|c|c|c|c|}
\hline $\begin{array}{c}\text { SITE } \\
\text { NUMBER } \\
\text { (figure 2) }\end{array}$ & DATE & $\begin{array}{l}\text { MAGNE- } \\
\text { SIUM, } \\
\text { DIS- } \\
\text { SOLVED } \\
\text { (MG/L } \\
\text { AS MG) }\end{array}$ & $\begin{array}{l}\text { SODIUM, } \\
\text { DIS- } \\
\text { SOLVED } \\
\text { (MG/L } \\
\text { AS NA) }\end{array}$ & $\begin{array}{l}\text { POTAS- } \\
\text { SIUM, } \\
\text { DIS- } \\
\text { SOLVED } \\
(\text { MG/L } \\
\text { AS K) }\end{array}$ & $\begin{array}{l}\text { BICAR- } \\
\text { BONATE, } \\
\text { IT-FIELD } \\
(\mathrm{MG} / \mathrm{L} \\
\left.\text { AS } \mathrm{HCO}_{3}\right)\end{array}$ & $\begin{array}{c}\text { CAR- } \\
\text { BONATE, } \\
\text { IT-FIELD } \\
(\mathrm{MG} / \mathrm{L} \\
\left.\text { AS } \mathrm{CO}_{3}\right)\end{array}$ & $\begin{array}{c}\text { ALKA- } \\
\text { LINITY, } \\
\text { CARBON- } \\
\text { ATE, } \\
\text { IT-FIELD } \\
\left(\mathrm{MG} / \mathrm{L}^{\mathrm{AS}}\right. \\
\left.\mathrm{CACO}_{3}\right)\end{array}$ \\
\hline 7 & $\begin{array}{l}12-03-85 \\
02-27-86 \\
05-09-86 \\
08-11-86\end{array}$ & $\begin{array}{l}11 \\
12 \\
12 \\
12\end{array}$ & $\begin{array}{l}52 \\
54 \\
53 \\
56\end{array}$ & $\begin{array}{l}1.9 \\
2.2 \\
2.3 \\
2.2\end{array}$ & $\begin{array}{l}217 \\
201 \\
205 \\
203\end{array}$ & $\begin{array}{l}0 \\
0 \\
0 \\
0\end{array}$ & $\begin{array}{l}178 \\
165 \\
168 \\
166\end{array}$ \\
\hline 8 & $\begin{array}{l}12-03-85 \\
02-26-86 \\
05-09-86 \\
08-19-86\end{array}$ & $\begin{array}{l}1.9 \\
2.0 \\
1.9 \\
1.8\end{array}$ & $\begin{array}{l}33 \\
33 \\
33 \\
33\end{array}$ & $\begin{array}{l}1.4 \\
1.6 \\
1.6 \\
1.6\end{array}$ & $\begin{array}{l}107 \\
104 \\
106 \\
105\end{array}$ & $\begin{array}{l}0 \\
0 \\
0 \\
0\end{array}$ & $\begin{array}{l}88 \\
85 \\
87 \\
86\end{array}$ \\
\hline 9 & $\begin{array}{l}11-22-85 \\
02-27-86 \\
05-09-86 \\
08-12-86\end{array}$ & $\begin{array}{r}.1 \\
.1 \\
.1 \\
<.01\end{array}$ & $\begin{array}{l}14 \\
14 \\
14 \\
14\end{array}$ & $\begin{array}{r}.9 \\
.8 \\
.9 \\
1.0\end{array}$ & $\begin{array}{l}70 \\
62 \\
66 \\
67\end{array}$ & $\begin{array}{l}0 \\
6 \\
2 \\
4\end{array}$ & $\begin{array}{l}58 \\
61 \\
58 \\
61\end{array}$ \\
\hline 10 & $\begin{array}{l}12-09-85 \\
02-10-86 \\
05-19-86 \\
08-13-86\end{array}$ & $\begin{array}{l}6.0 \\
5.0 \\
5.0 \\
5.0\end{array}$ & $\begin{array}{l}450 \\
470 \\
490 \\
510\end{array}$ & $\begin{array}{l}5.2 \\
5.6 \\
6.0 \\
6.0\end{array}$ & $\begin{array}{l}180 \\
162 \\
154 \\
159\end{array}$ & $\begin{array}{l}0 \\
0 \\
0 \\
0\end{array}$ & $\begin{array}{l}148 \\
133 \\
126 \\
130\end{array}$ \\
\hline 11 & $\begin{array}{l}11-20-85 \\
02-26-86 \\
05-20-86 \\
08-13-86\end{array}$ & $\begin{array}{l}3.5 \\
4.0 \\
3.6 \\
3.6\end{array}$ & $\begin{array}{l}58 \\
64 \\
59 \\
61\end{array}$ & $\begin{array}{l}3.8 \\
3.6 \\
3.8 \\
4.0\end{array}$ & $\begin{array}{l}190 \\
182 \\
178 \\
176\end{array}$ & $\begin{array}{l}0 \\
0 \\
0 \\
0\end{array}$ & $\begin{array}{l}160 \\
149 \\
146 \\
144\end{array}$ \\
\hline 12 & $\begin{array}{l}11-22-85 \\
02-12-86 \\
05-20-86 \\
08-13-86\end{array}$ & $\begin{array}{l}4.4 \\
4.6 \\
4.4 \\
4.7\end{array}$ & $\begin{array}{l}53 \\
54 \\
53 \\
54\end{array}$ & $\begin{array}{l}3.2 \\
3.5 \\
3.5 \\
3.7\end{array}$ & $\begin{array}{l}130 \\
128 \\
134 \\
131\end{array}$ & $\begin{array}{l}0 \\
0 \\
0 \\
0\end{array}$ & $\begin{array}{l}110 \\
105 \\
110 \\
107\end{array}$ \\
\hline 13 & $\begin{array}{l}11-22-85 \\
02-12-86 \\
05-20-86 \\
08-27-86\end{array}$ & $\begin{array}{l}3.0 \\
2.9 \\
2.9 \\
3.0\end{array}$ & $\begin{array}{l}60 \\
61 \\
58 \\
58\end{array}$ & $\begin{array}{l}4.0 \\
4.4 \\
4.4 \\
4.5\end{array}$ & $\begin{array}{l}140 \\
141 \\
140 \\
140\end{array}$ & $\begin{array}{l}0 \\
0 \\
0 \\
0\end{array}$ & $\begin{array}{l}120 \\
116 \\
115 \\
115\end{array}$ \\
\hline 14 & $\begin{array}{l}11-25-85 \\
02-06-86 \\
05-13-86 \\
08-18-86\end{array}$ & $\begin{array}{l}5.9 \\
5.2 \\
5.6 \\
5.6\end{array}$ & $\begin{array}{l}15 \\
14 \\
15 \\
15\end{array}$ & $\begin{array}{l}2.8 \\
2.9 \\
2.9 \\
2.9\end{array}$ & $\begin{array}{l}180 \\
180 \\
183 \\
183\end{array}$ & $\begin{array}{l}0 \\
0 \\
0 \\
0\end{array}$ & $\begin{array}{l}150 \\
148 \\
150 \\
150\end{array}$ \\
\hline
\end{tabular}


TABLE 5.--Data on physical properties and major chemical constituents--Continued

\begin{tabular}{|c|c|c|c|c|c|c|c|c|}
\hline $\begin{array}{c}\text { SITE } \\
\text { NUMBER } \\
\text { (figure 2) }\end{array}$ & DATE & $\begin{array}{l}\text { SULFATE, } \\
\text { DIS- } \\
\text { SOLVED } \\
\text { (MG/L } \\
\left.\text { AS } \mathrm{SO}_{4}\right)\end{array}$ & $\begin{array}{l}\text { CHLO- } \\
\text { RIDE, } \\
\text { DIS- } \\
\text { SOLVED } \\
\text { (MG/L } \\
\text { AS CL) }\end{array}$ & $\begin{array}{l}\text { FLUO- } \\
\text { RIDE, } \\
\text { DIS- } \\
\text { SOLVED } \\
\text { (MG/L } \\
\text { AS F) }\end{array}$ & $\begin{array}{l}\text { SILICA, } \\
\text { DIS- } \\
\text { SOLVED } \\
\text { (MG/L } \\
\mathrm{AS} \\
\mathrm{SIO}_{2} \text { ) }\end{array}$ & $\begin{array}{l}\text { SOLIDS, } \\
\text { RESIDUE } \\
\text { AT } 180 \\
\text { DEG C, } \\
\text { DIS- } \\
\text { SOLVED } \\
(\mathrm{MG} / \mathrm{L})\end{array}$ & $\begin{array}{l}\text { SOLIDS, } \\
\text { SUM OF } \\
\text { CONSTI- } \\
\text { TUENTS, } \\
\text { DIS- } \\
\text { SOLVED } \\
(\mathrm{MG} / \mathrm{L})\end{array}$ & $\begin{array}{c}\text { TUR- } \\
\text { BID- } \\
\text { ITY } \\
\text { (NTU) }\end{array}$ \\
\hline 7 & $\begin{array}{l}12-03-85 \\
02-27-86 \\
05-09-86 \\
08-11-86\end{array}$ & $\begin{array}{l}32 \\
34 \\
42 \\
44\end{array}$ & $\begin{array}{l}58 \\
59 \\
69 \\
69\end{array}$ & $\begin{array}{r}0.6 \\
.6 \\
.5 \\
.7\end{array}$ & $\begin{array}{l}54 \\
57 \\
55 \\
55\end{array}$ & $\begin{array}{l}472 \\
491 \\
507 \\
519\end{array}$ & $\begin{array}{l}390 \\
390 \\
410 \\
420\end{array}$ & $\begin{array}{r}0.6 \\
3.5 \\
.6 \\
.5\end{array}$ \\
\hline 8 & $\begin{array}{l}12-03-85 \\
02-26-86 \\
05-09-86 \\
08-19-86\end{array}$ & $\begin{array}{l}13 \\
16 \\
20 \\
19\end{array}$ & $\begin{array}{l}11 \\
13 \\
13 \\
12\end{array}$ & $\begin{array}{r}1.6 \\
1.7 \\
1.4 \\
1.4\end{array}$ & $\begin{array}{l}33 \\
35 \\
33 \\
31\end{array}$ & $\begin{array}{l}159 \\
155 \\
167 \\
168\end{array}$ & $\begin{array}{l}170 \\
170 \\
170 \\
170\end{array}$ & $\begin{array}{r}.4 \\
1.0 \\
.6 \\
.6\end{array}$ \\
\hline 9 & $\begin{array}{l}11-22-85 \\
02-27-86 \\
05-09-86 \\
08-12-86\end{array}$ & $\begin{array}{r}.8 \\
.9 \\
.7 \\
1.1\end{array}$ & $\begin{array}{l}1.0 \\
1.1 \\
1.3 \\
1.0\end{array}$ & $\begin{array}{l}.1 \\
<.1 \\
<.1 \\
<.1\end{array}$ & $\begin{array}{l}17 \\
18 \\
17 \\
17\end{array}$ & $\begin{array}{l}82 \\
85 \\
81 \\
78\end{array}$ & $\begin{array}{l}81 \\
91 \\
84 \\
--\end{array}$ & $\begin{array}{r}.7 \\
.4 \\
.8 \\
-.\end{array}$ \\
\hline 10 & $\begin{array}{l}12-09-85 \\
02-10-86 \\
05-19-86 \\
08-13-86\end{array}$ & $\begin{array}{l}1400 \\
1300 \\
1300 \\
1400\end{array}$ & $\begin{array}{r}97 \\
93 \\
110 \\
150\end{array}$ & $\begin{array}{l}-- \\
5.2 \\
5.4 \\
5.2\end{array}$ & $\begin{array}{l}54 \\
53 \\
58 \\
56\end{array}$ & $\begin{array}{l}-- \\
2310 \\
2390 \\
2430\end{array}$ & $\begin{array}{l}2300 \\
2200 \\
2300 \\
2400\end{array}$ & $\begin{array}{r}84 \\
95 \\
60 \\
120\end{array}$ \\
\hline 11 & $\begin{array}{l}11-20-85 \\
02-26-86 \\
05-20-86 \\
08-13-86\end{array}$ & $\begin{array}{l}69 \\
72 \\
70 \\
81\end{array}$ & $\begin{array}{l}16 \\
13 \\
13 \\
12\end{array}$ & $\begin{array}{l}1.6 \\
1.9 \\
1.8 \\
1.7\end{array}$ & $\begin{array}{l}57 \\
63 \\
60 \\
58\end{array}$ & $\begin{array}{l}321 \\
337 \\
338 \\
337\end{array}$ & $\begin{array}{l}340 \\
350 \\
330 \\
340\end{array}$ & $\begin{array}{r}1.6 \\
.5 \\
.6 \\
.3\end{array}$ \\
\hline 12 & $\begin{array}{l}11-22-85 \\
02-12-86 \\
05-20-86 \\
08-13-86\end{array}$ & $\begin{array}{l}47 \\
43 \\
45 \\
50\end{array}$ & $\begin{array}{l}11 \\
10 \\
11 \\
13\end{array}$ & $\begin{array}{l}1.6 \\
1.6 \\
1.7 \\
1.5\end{array}$ & $\begin{array}{l}64 \\
65 \\
65 \\
63\end{array}$ & $\begin{array}{l}266 \\
258 \\
268 \\
277\end{array}$ & $\begin{array}{l}270 \\
260 \\
260 \\
270\end{array}$ & $\begin{array}{r}1.0 \\
1.3 \\
.5 \\
.2\end{array}$ \\
\hline 13 & $\begin{array}{l}11-22-85 \\
02-12-86 \\
05-20-86 \\
08-27-86\end{array}$ & $\begin{array}{l}33 \\
31 \\
36 \\
34\end{array}$ & $\begin{array}{l}9.4 \\
9.7 \\
9.5 \\
8.9\end{array}$ & $\begin{array}{l}1.5 \\
1.6 \\
1.6 \\
1.5\end{array}$ & $\begin{array}{l}78 \\
80 \\
78 \\
75\end{array}$ & $\begin{array}{l}264 \\
276 \\
271 \\
262\end{array}$ & $\begin{array}{l}270 \\
270 \\
270 \\
260\end{array}$ & $\begin{array}{r}1.0 \\
.3 \\
1.0 \\
-.\end{array}$ \\
\hline 14 & $\begin{array}{l}11-25-85 \\
02-06-86 \\
05-13-86 \\
08-18-86\end{array}$ & $\begin{array}{l}11 \\
11 \\
11 \\
12\end{array}$ & $\begin{array}{l}1.2 \\
1.2 \\
1.0 \\
1.3\end{array}$ & $\begin{array}{l}.2 \\
.1 \\
.1 \\
.2\end{array}$ & $\begin{array}{l}22 \\
20 \\
22 \\
22\end{array}$ & $\begin{array}{l}174 \\
174 \\
184 \\
186\end{array}$ & $\begin{array}{l}190 \\
180 \\
190 \\
190\end{array}$ & $\begin{array}{l}1.2 \\
1.4 \\
1.0 \\
1.1\end{array}$ \\
\hline
\end{tabular}


TABLE 5.--Data on physical properties and major chemical constituents--Continued

\begin{tabular}{|c|c|c|c|c|c|c|c|c|}
\hline $\begin{array}{c}\text { SITE } \\
\text { NUMBER } \\
\text { (figure 2) }\end{array}$ & DATE & TIME & $\begin{array}{l}\text { FIELD } \\
\text { SPE- } \\
\text { CIFIC } \\
\text { CON- } \\
\text { DUCT- } \\
\text { ANCE } \\
\text { (US/CM) }\end{array}$ & $\begin{array}{c}\text { FIELD } \\
\text { pH } \\
\text { (STAND- } \\
\text { ARD } \\
\text { UNITS) }\end{array}$ & $\begin{array}{c}\text { WATER } \\
\text { TEMPER- } \\
\text { ATURE } \\
\text { (DEG C) }\end{array}$ & $\begin{array}{c}\text { TOTAL } \\
\text { HARD- } \\
\text { NESS } \\
\text { (MG/L } \\
\text { AS } \\
\left.\mathrm{CACO}_{3}\right)\end{array}$ & $\begin{array}{l}\text { HARD- } \\
\text { NESS, } \\
\text { NONCAR- } \\
\text { BONATE } \\
(\mathrm{MG} / \mathrm{L} \\
\mathrm{AS} \\
\left.\mathrm{CACO}_{3}\right)\end{array}$ & $\begin{array}{l}\text { CALCIUM, } \\
\text { DIS- } \\
\text { SOLVED } \\
\text { (MG/L } \\
\text { AS CA) }\end{array}$ \\
\hline 15 & $\begin{array}{l}11-20-85 \\
02-10-86 \\
05-13-86\end{array}$ & $\begin{array}{l}1235 \\
1430 \\
1610\end{array}$ & $\begin{array}{l}375 \\
310 \\
290\end{array}$ & $\begin{array}{l}7.8 \\
8.1 \\
8.2\end{array}$ & $\begin{array}{l}14.5 \\
14.0 \\
14.5\end{array}$ & $\begin{array}{l}93 \\
83 \\
72\end{array}$ & $\begin{array}{r}10 \\
5 \\
5\end{array}$ & $\begin{array}{l}34 \\
30 \\
27\end{array}$ \\
\hline 16 & $\begin{array}{l}11-27-85 \\
02-06-86 \\
05-13-86 \\
08-18-86\end{array}$ & $\begin{array}{l}1245 \\
1235 \\
1350 \\
1140\end{array}$ & $\begin{array}{l}220 \\
210 \\
225 \\
215\end{array}$ & $\begin{array}{l}7.4 \\
7.6 \\
7.6 \\
7.4\end{array}$ & $\begin{array}{l}12.5 \\
12.5 \\
12.5 \\
12.5\end{array}$ & $\begin{array}{l}88 \\
88 \\
88 \\
90\end{array}$ & $\begin{array}{l}0 \\
0 \\
0 \\
0\end{array}$ & $\begin{array}{l}26 \\
26 \\
26 \\
27\end{array}$ \\
\hline 17 & $\begin{array}{l}11-25-85 \\
02-06-86 \\
05-13-86 \\
08-18-86\end{array}$ & $\begin{array}{l}1030 \\
1435 \\
1010 \\
1000\end{array}$ & $\begin{array}{l}150 \\
195 \\
215 \\
185\end{array}$ & $\begin{array}{l}7.9 \\
8.1 \\
8.2 \\
8.0\end{array}$ & $\begin{array}{l}16.0 \\
15.5 \\
15.5 \\
15.5\end{array}$ & $\begin{array}{l}37 \\
51 \\
54 \\
47\end{array}$ & $\begin{array}{l}0 \\
0 \\
0 \\
0\end{array}$ & $\begin{array}{l}10 \\
14 \\
15 \\
13\end{array}$ \\
\hline 18 & $\begin{array}{l}11-26-85 \\
02-25-86 \\
05-19-86 \\
08-26-86\end{array}$ & $\begin{array}{l}1605 \\
1530 \\
1510 \\
1050\end{array}$ & $\begin{array}{l}330 \\
315 \\
320 \\
325\end{array}$ & $\begin{array}{l}7.5 \\
7.7 \\
7.6 \\
7.8\end{array}$ & $\begin{array}{l}15.5 \\
15.5 \\
16.0 \\
15.5\end{array}$ & $\begin{array}{l}110 \\
120 \\
110 \\
110\end{array}$ & $\begin{array}{l}0 \\
0 \\
0 \\
0\end{array}$ & $\begin{array}{l}31 \\
31 \\
29 \\
30\end{array}$ \\
\hline 19 & $\begin{array}{l}12-10-85 \\
02-11-86 \\
05-14-86 \\
08-12-86\end{array}$ & $\begin{array}{l}1305 \\
1250 \\
1240 \\
1250\end{array}$ & $\begin{array}{l}485 \\
465 \\
505 \\
440\end{array}$ & $\begin{array}{l}7.7 \\
8.0 \\
7.9 \\
8.0\end{array}$ & $\begin{array}{l}11.0 \\
11.5 \\
12.0 \\
12.5\end{array}$ & $\begin{array}{l}220 \\
220 \\
210 \\
220\end{array}$ & $\begin{array}{l}0 \\
0 \\
0 \\
0\end{array}$ & $\begin{array}{l}60 \\
62 \\
60 \\
62\end{array}$ \\
\hline 20 & $\begin{array}{l}11-13-85 \\
02-07-86 \\
05-16-86 \\
08-21-86\end{array}$ & $\begin{array}{l}1400 \\
1315 \\
1240 \\
1535\end{array}$ & $\begin{array}{l}325 \\
310 \\
315 \\
325\end{array}$ & $\begin{array}{l}7.6 \\
7.8 \\
7.8 \\
7.7\end{array}$ & $\begin{array}{l}14.0 \\
13.0 \\
14.5 \\
14.5\end{array}$ & $\begin{array}{l}110 \\
110 \\
100 \\
120\end{array}$ & $\begin{array}{l}0 \\
0 \\
0 \\
0\end{array}$ & $\begin{array}{l}33 \\
32 \\
30 \\
35\end{array}$ \\
\hline 21 & $\begin{array}{l}11-20-85 \\
02-07-86 \\
05-15-86 \\
08-21-86\end{array}$ & $\begin{array}{l}1030 \\
1040 \\
1410 \\
1340\end{array}$ & $\begin{array}{l}265 \\
285 \\
285 \\
265\end{array}$ & $\begin{array}{l}7.7 \\
8.0 \\
8.0 \\
8.0\end{array}$ & $\begin{array}{l}15.0 \\
13.0 \\
14.0 \\
15.5\end{array}$ & $\begin{array}{l}100 \\
110 \\
100 \\
100\end{array}$ & $\begin{array}{l}2 \\
1 \\
0 \\
0\end{array}$ & $\begin{array}{l}31 \\
33 \\
31 \\
31\end{array}$ \\
\hline 22 & $\begin{array}{l}11-26-85 \\
02-11-86 \\
05-14-86 \\
08-21-86\end{array}$ & $\begin{array}{l}1325 \\
1500 \\
1630 \\
1000\end{array}$ & $\begin{array}{l}200 \\
210 \\
225 \\
205\end{array}$ & $\begin{array}{l}6.7 \\
7.0 \\
7.0 \\
7.1\end{array}$ & $\begin{array}{l}14.0 \\
13.5 \\
13.5 \\
14.0\end{array}$ & $\begin{array}{l}83 \\
81 \\
85 \\
80\end{array}$ & $\begin{array}{l}1 \\
0 \\
0 \\
0\end{array}$ & $\begin{array}{l}24 \\
19 \\
20 \\
19\end{array}$ \\
\hline
\end{tabular}


TABLE 5.--Data on physical properties and major chemical constituents--Continued

\begin{tabular}{|c|c|c|c|c|c|c|c|}
\hline $\begin{array}{c}\text { SITE } \\
\text { NUMBER } \\
\text { (figure 2) }\end{array}$ & DATE & $\begin{array}{l}\text { MAGNE- } \\
\text { SIUM, } \\
\text { DIS- } \\
\text { SOLVED } \\
\text { (MG/L } \\
\text { AS MG) }\end{array}$ & $\begin{array}{l}\text { SODIUM, } \\
\text { DIS- } \\
\text { SOLVED } \\
\text { (MG/L } \\
\text { AS NA) }\end{array}$ & $\begin{array}{l}\text { POTAS- } \\
\text { SIUM, } \\
\text { DIS- } \\
\text { SOLVED } \\
\text { (MG/L } \\
\text { AS K) }\end{array}$ & $\begin{array}{l}\text { BICAR- } \\
\text { BONATE, } \\
\text { IT-FIELD } \\
(\mathrm{MG} / \mathrm{L} \\
\left.\text { AS } \mathrm{HCO}_{3}\right)\end{array}$ & $\begin{array}{c}\text { CAR- } \\
\text { BONATE, } \\
\text { IT-FIELD } \\
(\mathrm{MG} / \mathrm{L} \\
\left.\text { AS } \mathrm{CO}_{3}\right)\end{array}$ & $\begin{array}{l}\text { ALKA- } \\
\text { LINITY, } \\
\text { CARBON- } \\
\text { ATE, } \\
\text { IT-FIELD } \\
(\mathrm{MG} / \mathrm{L} \text { AS } \\
\left.\mathrm{CACO}_{3}\right)\end{array}$ \\
\hline 15 & $\begin{array}{l}11-20-85 \\
02-10-86 \\
05-13-86\end{array}$ & $\begin{array}{l}2.0 \\
1.9 \\
1.1\end{array}$ & $\begin{array}{l}35 \\
30 \\
24\end{array}$ & $\begin{array}{l}3.4 \\
3.2 \\
3.2\end{array}$ & $\begin{array}{r}100 \\
95 \\
81\end{array}$ & $\begin{array}{l}0 \\
0 \\
0\end{array}$ & $\begin{array}{l}83 \\
78 \\
67\end{array}$ \\
\hline 16 & $\begin{array}{l}11-27-85 \\
02-06-86 \\
05-13-86 \\
08-18-86\end{array}$ & $\begin{array}{l}5.7 \\
5.5 \\
5.6 \\
5.6\end{array}$ & $\begin{array}{l}10 \\
9.7 \\
10 \\
10\end{array}$ & $\begin{array}{l}1.5 \\
1.3 \\
1.3 \\
1.2\end{array}$ & $\begin{array}{l}132 \\
130 \\
127 \\
126\end{array}$ & $\begin{array}{l}0 \\
0 \\
0 \\
0\end{array}$ & $\begin{array}{l}108 \\
107 \\
104 \\
103\end{array}$ \\
\hline 17 & $\begin{array}{l}11-25-85 \\
02-06-86 \\
05-13-86 \\
08-18-86\end{array}$ & $\begin{array}{l}3.0 \\
3.8 \\
4.1 \\
3.6\end{array}$ & $\begin{array}{l}15 \\
17 \\
17 \\
16\end{array}$ & $\begin{array}{l}4.2 \\
4.4 \\
4.4 \\
4.4\end{array}$ & $\begin{array}{l}74 \\
89 \\
90 \\
85\end{array}$ & $\begin{array}{l}0 \\
0 \\
0 \\
0\end{array}$ & $\begin{array}{l}61 \\
73 \\
74 \\
70\end{array}$ \\
\hline 18 & $\begin{array}{l}11-26-85 \\
02-25-86 \\
05-19-86 \\
08-26-86\end{array}$ & $\begin{array}{l}9.1 \\
9.3 \\
9.0 \\
8.9\end{array}$ & $\begin{array}{l}25 \\
26 \\
24 \\
25\end{array}$ & $\begin{array}{l}3.1 \\
3.3 \\
3.2 \\
3.2\end{array}$ & $\begin{array}{l}160 \\
159 \\
160 \\
160\end{array}$ & $\begin{array}{l}0 \\
0 \\
0 \\
0\end{array}$ & $\begin{array}{l}130 \\
130 \\
131 \\
131\end{array}$ \\
\hline 19 & $\begin{array}{l}12-10-85 \\
02-11-86 \\
05-14-86 \\
08-12-86\end{array}$ & $\begin{array}{l}16 \\
15 \\
15 \\
15\end{array}$ & $\begin{array}{l}20 \\
19 \\
18 \\
19\end{array}$ & $\begin{array}{l}3.4 \\
3.6 \\
3.6 \\
3.5\end{array}$ & $\begin{array}{l}280 \\
281 \\
281 \\
283\end{array}$ & $\begin{array}{l}0 \\
0 \\
0 \\
0\end{array}$ & $\begin{array}{l}230 \\
230 \\
230 \\
232\end{array}$ \\
\hline 20 & $\begin{array}{l}11-13-85 \\
02-07-86 \\
05-16-86 \\
08-21-86\end{array}$ & $\begin{array}{l}7.8 \\
7.2 \\
7.0 \\
8.1\end{array}$ & $\begin{array}{l}25 \\
23 \\
21 \\
23\end{array}$ & $\begin{array}{l}1.8 \\
1.6 \\
1.7 \\
1.7\end{array}$ & $\begin{array}{l}180 \\
167 \\
162 \\
187\end{array}$ & $\begin{array}{l}0 \\
0 \\
0 \\
0\end{array}$ & $\begin{array}{l}150 \\
137 \\
133 \\
153\end{array}$ \\
\hline 21 & $\begin{array}{l}11-20-85 \\
02-07-86 \\
05-15-86 \\
08-21-86\end{array}$ & $\begin{array}{l}6.0 \\
6.2 \\
6.0 \\
5.8\end{array}$ & $\begin{array}{l}14 \\
15 \\
15 \\
14\end{array}$ & $\begin{array}{l}2.4 \\
2.4 \\
2.4 \\
2.3\end{array}$ & $\begin{array}{l}130 \\
130 \\
127 \\
127\end{array}$ & $\begin{array}{l}0 \\
0 \\
0 \\
0\end{array}$ & $\begin{array}{l}100 \\
107 \\
104 \\
104\end{array}$ \\
\hline 22 & $\begin{array}{l}11-26-85 \\
02-11-86 \\
05-14-86 \\
08-21-86\end{array}$ & $\begin{array}{l}5.7 \\
8.1 \\
8.6 \\
7.9\end{array}$ & $\begin{array}{l}15 \\
9.6 \\
9.5 \\
9.4\end{array}$ & $\begin{array}{l}2.5 \\
2.6 \\
2.7 \\
2.5\end{array}$ & $\begin{array}{l}100 \\
104 \\
110 \\
103\end{array}$ & $\begin{array}{l}0 \\
0 \\
0 \\
0\end{array}$ & $\begin{array}{l}82 \\
85 \\
90 \\
84\end{array}$ \\
\hline
\end{tabular}


TABLE 5.--Data on physical properties and major chemical constituents--Continued

\begin{tabular}{|c|c|c|c|c|c|c|c|c|}
\hline $\begin{array}{c}\text { SITE } \\
\text { NUMBER } \\
\text { (f1gure 2) }\end{array}$ & DATE & $\begin{array}{l}\text { SULFATE, } \\
\text { DIS- } \\
\text { SOLVED } \\
(\mathrm{MG} / \mathrm{L} \\
\left.\mathrm{AS} \mathrm{SO}_{4}\right)\end{array}$ & $\begin{array}{l}\text { CHLO- } \\
\text { RIDE, } \\
\text { DIS- } \\
\text { SOLVED } \\
\text { (MG/L } \\
\text { AS CL) }\end{array}$ & $\begin{array}{l}\text { FLUO- } \\
\text { RIDE, } \\
\text { DIS- } \\
\text { SOLVED } \\
(M G / L \\
\text { AS F) }\end{array}$ & $\begin{array}{l}\text { SILICA, } \\
\text { DIS- } \\
\text { SOLVED } \\
(\mathrm{MG} / \mathrm{L} \\
\mathrm{AS} \\
\left.\mathrm{SIO}_{2}\right)\end{array}$ & $\begin{array}{l}\text { SOLIDS, } \\
\text { RESIDUE } \\
\text { AT } 180 \\
\text { DEG C, } \\
\text { DIS- } \\
\text { SOLVED } \\
(\mathrm{MG} / \mathrm{L})\end{array}$ & $\begin{array}{l}\text { SOLIDS, } \\
\text { SUM OF } \\
\text { CONSTI- } \\
\text { TUENTS, } \\
\text { DIS- } \\
\text { SOLVED } \\
\text { (MG/L) }\end{array}$ & $\begin{array}{c}\text { TUR- } \\
\text { BID- } \\
\text { ITY } \\
\text { (NTU) }\end{array}$ \\
\hline 15 & $\begin{array}{l}11-20-85 \\
02-10-86 \\
05-13-86\end{array}$ & $\begin{array}{l}70 \\
53 \\
43\end{array}$ & $\begin{array}{l}20 \\
14 \\
13\end{array}$ & $\begin{array}{r}0.4 \\
.5 \\
.5\end{array}$ & $\begin{array}{l}51 \\
53 \\
54\end{array}$ & $\begin{array}{l}277 \\
228 \\
205\end{array}$ & $\begin{array}{l}270 \\
230 \\
210\end{array}$ & $\begin{array}{r}0.5 \\
.7 \\
.6\end{array}$ \\
\hline 16 & $\begin{array}{l}11-27-85 \\
02-06-86 \\
05-13-86 \\
08-18-86\end{array}$ & $\begin{array}{l}3.4 \\
2.7 \\
2.2 \\
5.2\end{array}$ & $\begin{array}{l}1.6 \\
2.1 \\
2.9 \\
2.1\end{array}$ & $\begin{array}{l}<.1 \\
<.1 \\
<.1 \\
<.1\end{array}$ & $\begin{array}{l}23 \\
22 \\
23 \\
23\end{array}$ & $\begin{array}{l}132 \\
126 \\
133 \\
136\end{array}$ & $\begin{array}{l}140 \\
130 \\
130 \\
140\end{array}$ & $\begin{array}{r}1.0 \\
.8 \\
.5 \\
2.7\end{array}$ \\
\hline 17 & $\begin{array}{l}11-25-85 \\
02-06-86 \\
05-13-86 \\
08-18-86\end{array}$ & $\begin{array}{l}14 \\
14 \\
14 \\
15\end{array}$ & $\begin{array}{l}2.8 \\
4.3 \\
4.0 \\
3.7\end{array}$ & $\begin{array}{l}.4 \\
.3 \\
.3 \\
.3\end{array}$ & $\begin{array}{l}49 \\
48 \\
49 \\
49\end{array}$ & $\begin{array}{l}138 \\
143 \\
151 \\
146\end{array}$ & $\begin{array}{l}140 \\
150 \\
150 \\
150\end{array}$ & $\begin{array}{l}.5 \\
.5 \\
.6 \\
.2\end{array}$ \\
\hline 18 & $\begin{array}{l}11-26-85 \\
02-25-86 \\
05-19-86 \\
08-26-86\end{array}$ & $\begin{array}{l}34 \\
23 \\
30 \\
29\end{array}$ & $\begin{array}{l}6.8 \\
7.7 \\
7.7 \\
7.1\end{array}$ & $\begin{array}{l}.2 \\
.2 \\
.2 \\
.3\end{array}$ & $\begin{array}{l}50 \\
53 \\
51 \\
49\end{array}$ & $\begin{array}{l}224 \\
231 \\
238 \\
214\end{array}$ & $\begin{array}{l}240 \\
230 \\
230 \\
230\end{array}$ & $\begin{array}{r}1.0 \\
.4 \\
1.0 \\
-\end{array}$ \\
\hline 19 & $\begin{array}{l}12-10-85 \\
02-11-86 \\
05-14-86 \\
08-12-86\end{array}$ & $\begin{array}{l}14 \\
15 \\
18 \\
20\end{array}$ & $\begin{array}{l}5.8 \\
5.6 \\
5.6 \\
5.6\end{array}$ & $\begin{array}{l}.1 \\
<.1 \\
<.1 \\
<.1\end{array}$ & $\begin{array}{l}31 \\
31 \\
31 \\
32\end{array}$ & $\begin{array}{l}286 \\
285 \\
292 \\
302\end{array}$ & $\begin{array}{l}290 \\
290 \\
290 \\
300\end{array}$ & $\begin{array}{r}1.3 \\
.6 \\
.8 \\
-\end{array}$ \\
\hline 20 & $\begin{array}{l}11-13-85 \\
02-07-86 \\
05-16-86 \\
08-21-86\end{array}$ & $\begin{array}{l}17 \\
15 \\
18 \\
16\end{array}$ & $\begin{array}{l}4.0 \\
4.3 \\
4.1 \\
3.7\end{array}$ & $\begin{array}{l}.2 \\
.2 \\
.2 \\
.3\end{array}$ & $\begin{array}{l}36 \\
36 \\
37 \\
36\end{array}$ & $\begin{array}{l}208 \\
193 \\
197 \\
206\end{array}$ & $\begin{array}{l}210 \\
200 \\
200 \\
220\end{array}$ & $\begin{array}{l}.8 \\
.4 \\
.5 \\
.-\end{array}$ \\
\hline 21 & $\begin{array}{l}11-20-85 \\
02-07-86 \\
05-15-86 \\
08-21-86\end{array}$ & $\begin{array}{l}22 \\
16 \\
21 \\
21\end{array}$ & $\begin{array}{l}11 \\
8.8 \\
8.7 \\
8.3\end{array}$ & $\begin{array}{r}<.1 \\
<.1 \\
.2 \\
<.1\end{array}$ & $\begin{array}{l}30 \\
31 \\
32 \\
30\end{array}$ & $\begin{array}{l}180 \\
174 \\
180 \\
172\end{array}$ & $\begin{array}{l}180 \\
180 \\
180 \\
170\end{array}$ & $\begin{array}{r}1.1 \\
.4 \\
1.0 \\
--\end{array}$ \\
\hline 22 & $\begin{array}{l}11-26-85 \\
02-11-86 \\
05-14-86 \\
08-21-86\end{array}$ & $\begin{array}{l}12 \\
11 \\
12 \\
10\end{array}$ & $\begin{array}{l}4.3 \\
5.2 \\
6.0 \\
4.2\end{array}$ & $\begin{array}{l}<.1 \\
<.1 \\
.1 \\
.1\end{array}$ & $\begin{array}{l}33 \\
45 \\
47 \\
45\end{array}$ & $\begin{array}{l}144 \\
148 \\
162 \\
153\end{array}$ & $\begin{array}{l}150 \\
150 \\
160 \\
150\end{array}$ & $\begin{array}{r}1.5 \\
.9 \\
.6 \\
-\end{array}$ \\
\hline
\end{tabular}


TABLE 5.--Data on physical properties and major chemical constituents - - Continued

\begin{tabular}{|c|c|c|c|c|c|c|c|c|}
\hline $\begin{array}{c}\text { SITE } \\
\text { NUMBER } \\
\text { (f1gure 2) }\end{array}$ & DATE & TIME & $\begin{array}{l}\text { FIELD } \\
\text { SPE- } \\
\text { CIFIC } \\
\text { CON- } \\
\text { DUCT- } \\
\text { ANCE } \\
\text { (US/CM) }\end{array}$ & $\begin{array}{c}\text { FIELD } \\
\text { pH } \\
\text { (STAND- } \\
\text { ARD } \\
\text { UNITS ) }\end{array}$ & $\begin{array}{c}\text { WATER } \\
\text { TEMPER- } \\
\text { ATURE } \\
\text { (DEG C) }\end{array}$ & $\begin{array}{c}\text { TOTAL } \\
\text { HARD- } \\
\text { NESS } \\
(\mathrm{MG} / \mathrm{L} \\
\mathrm{AS}^{-} \\
\left.\mathrm{CACO}_{3}\right)\end{array}$ & $\begin{array}{c}\text { HARD- } \\
\text { NESS , } \\
\text { NONCAR- } \\
\text { BONATE } \\
\text { (MG/L } \\
\text { AS } \\
\mathrm{CACO}_{3} \text { ) }\end{array}$ & $\begin{array}{l}\text { CALCIUM, } \\
\text { DIS- } \\
\text { SOLVED } \\
\text { (MG/L } \\
\text { AS CA) }\end{array}$ \\
\hline 23 & $\begin{array}{l}11-19-85 \\
02-25-86 \\
05-15-86 \\
08-21-86\end{array}$ & $\begin{array}{l}1420 \\
1240 \\
1200 \\
1210\end{array}$ & $\begin{array}{l}560 \\
530 \\
580 \\
535\end{array}$ & $\begin{array}{l}7.7 \\
8.0 \\
7.9 \\
8.0\end{array}$ & $\begin{array}{l}13.5 \\
14.0 \\
14.0 \\
13.5\end{array}$ & $\begin{array}{l}230 \\
240 \\
240 \\
230\end{array}$ & $\begin{array}{l}27 \\
41 \\
42 \\
42\end{array}$ & $\begin{array}{l}71 \\
74 \\
74 \\
72\end{array}$ \\
\hline 24 & $\begin{array}{l}11-14-85 \\
02-25-86 \\
05-14-86 \\
08-12-86\end{array}$ & $\begin{array}{l}1510 \\
1015 \\
1745 \\
1000\end{array}$ & $\begin{array}{l}110 \\
105 \\
115 \\
105\end{array}$ & $\begin{array}{l}7.3 \\
7.5 \\
7.7 \\
7.8\end{array}$ & $\begin{array}{l}15.0 \\
14.5 \\
14.0 \\
14.5\end{array}$ & $\begin{array}{l}15 \\
14 \\
15 \\
15\end{array}$ & $\begin{array}{l}0 \\
0 \\
0 \\
0\end{array}$ & $\begin{array}{l}5.3 \\
5.0 \\
5.2 \\
5.2\end{array}$ \\
\hline 25 & $\begin{array}{l}11-14-85 \\
02-24-86 \\
05-12-86 \\
08-01-86\end{array}$ & $\begin{array}{l}1215 \\
1200 \\
1235 \\
1100\end{array}$ & $\begin{array}{l}485 \\
540 \\
525 \\
510\end{array}$ & $\begin{array}{l}7.1 \\
7.3 \\
7.2 \\
7.2\end{array}$ & $\begin{array}{l}17.0 \\
16.5 \\
17.0 \\
16.5\end{array}$ & $\begin{array}{l}220 \\
220 \\
210 \\
220\end{array}$ & $\begin{array}{l}63 \\
56 \\
49 \\
58\end{array}$ & $\begin{array}{l}63 \\
60 \\
59 \\
61\end{array}$ \\
\hline 26 & $\begin{array}{l}11-15-85 \\
02-24-86 \\
05-12-86 \\
08-01-86\end{array}$ & $\begin{array}{l}1335 \\
1400 \\
1430 \\
1310\end{array}$ & $\begin{array}{l}550 \\
440 \\
435 \\
445\end{array}$ & $\begin{array}{l}6.8 \\
7.1 \\
7.1 \\
7.1\end{array}$ & $\begin{array}{l}14.5 \\
14.5 \\
15.0 \\
14.5\end{array}$ & $\begin{array}{l}230 \\
190 \\
180 \\
210\end{array}$ & $\begin{array}{r}25 \\
11 \\
0 \\
13\end{array}$ & $\begin{array}{l}61 \\
48 \\
47 \\
54\end{array}$ \\
\hline 27 & $09-02-86$ & 1545 & 425 & 7.6 & 15.0 & 130 & 0 & 41 \\
\hline 28 & $\begin{array}{l}08-26-86 \\
08-26-86\end{array}$ & $\begin{array}{l}1305 \\
1400\end{array}$ & $\begin{array}{l}585 \\
585\end{array}$ & $\begin{array}{l}7.9 \\
7.9\end{array}$ & $\begin{array}{l}14.0 \\
13.0\end{array}$ & $\overline{94}$ & -- & 24 \\
\hline 29 & $08-19-86$ & 1525 & 605 & 6.8 & 17.0 & - & - & -- \\
\hline 30 & $08-19-86$ & 1430 & 205 & 8.5 & 18.5 & 23 & 0 & 8.2 \\
\hline 31 & $09-04-86$ & 1340 & 875 & 7.4 & 13.0 & 340 & 83 & 100 \\
\hline 32 & $09-05-86$ & 1445 & 645 & 7.4 & 13.5 & 240 & 0 & 66 \\
\hline 33 & $11-26-85$ & 1055 & 235 & 7.7 & 13.5 & 81 & 0 & 23 \\
\hline
\end{tabular}


TABLE 5.--Data on physical properties and major chemical const Ituents--Continued

\begin{tabular}{|c|c|c|c|c|c|c|c|}
\hline $\begin{array}{c}\text { SITE } \\
\text { NUMBER } \\
\text { ( f igure 2) }\end{array}$ & DATE & $\begin{array}{l}\text { MAGNE- } \\
\text { SIUM, } \\
\text { DIS- } \\
\text { SOLVED } \\
\text { (MG/L } \\
\text { AS MG) }\end{array}$ & $\begin{array}{l}\text { SODIUM, } \\
\text { DIS- } \\
\text { SOLVED } \\
\text { (MG/L } \\
\text { AS NA) }\end{array}$ & $\begin{array}{l}\text { POTAS- } \\
\text { SIUM, } \\
\text { DIS- } \\
\text { SOLVED } \\
\text { (MG/L } \\
\text { AS K) }\end{array}$ & $\begin{array}{c}\text { BICAR- } \\
\text { BONATE, } \\
\text { IT-FIELD } \\
(\mathrm{MG} / \mathrm{L} \\
\left.\text { AS } \mathrm{HCO}_{3}\right)\end{array}$ & $\begin{array}{c}\text { CAR- } \\
\text { BONATE, } \\
\text { IT-FIELD } \\
(\mathrm{MG} / \mathrm{L} \\
\left.\text { AS } \mathrm{CO}_{3}\right)\end{array}$ & 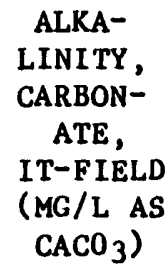 \\
\hline 23 & $\begin{array}{l}11-19-85 \\
02-25-86 \\
05-15-86 \\
08-21-86\end{array}$ & $\begin{array}{l}12 \\
14 \\
13 \\
13\end{array}$ & $\begin{array}{l}24 \\
26 \\
25 \\
23\end{array}$ & $\begin{array}{l}2.6 \\
2.6 \\
2.7 \\
2.5\end{array}$ & $\begin{array}{l}240 \\
245 \\
239 \\
233\end{array}$ & $\begin{array}{l}0 \\
0 \\
0 \\
0\end{array}$ & $\begin{array}{l}200 \\
201 \\
196 \\
191\end{array}$ \\
\hline 24 & $\begin{array}{l}11-14-85 \\
02-25-86 \\
05-14-86 \\
08-12-86\end{array}$ & $\begin{array}{l}.5 \\
.5 \\
.4 \\
.4\end{array}$ & $\begin{array}{l}18 \\
17 \\
16 \\
17\end{array}$ & $\begin{array}{r}1.0 \\
.9 \\
.8 \\
.9\end{array}$ & $\begin{array}{l}33 \\
37 \\
36 \\
39\end{array}$ & $\begin{array}{l}0 \\
0 \\
0 \\
0\end{array}$ & $\begin{array}{l}27 \\
30 \\
30 \\
32\end{array}$ \\
\hline 25 & $\begin{array}{l}11-14-85 \\
02-24-86 \\
05-12-86 \\
08-01-86\end{array}$ & $\begin{array}{l}16 \\
16 \\
15 \\
16\end{array}$ & $\begin{array}{l}23 \\
23 \\
22 \\
23\end{array}$ & $\begin{array}{l}1.6 \\
1.7 \\
1.7 \\
1.6\end{array}$ & $\begin{array}{l}200 \\
195 \\
195 \\
195\end{array}$ & $\begin{array}{l}0 \\
0 \\
0 \\
0\end{array}$ & $\begin{array}{l}160 \\
160 \\
160 \\
160\end{array}$ \\
\hline 26 & $\begin{array}{l}11-15-85 \\
02-24-86 \\
05-12-86 \\
08-01-86\end{array}$ & $\begin{array}{l}20 \\
17 \\
16 \\
18\end{array}$ & $\begin{array}{l}15 \\
13 \\
13 \\
13\end{array}$ & $\begin{array}{l}6.0 \\
5.6 \\
5.6 \\
5.8\end{array}$ & $\begin{array}{l}260 \\
218 \\
223 \\
239\end{array}$ & $\begin{array}{l}0 \\
0 \\
0 \\
0\end{array}$ & $\begin{array}{l}210 \\
179 \\
183 \\
196\end{array}$ \\
\hline 27 & $09-02-86$ & 7.4 & 35 & 5.5 & 177 & 0 & 145 \\
\hline 28 & $\begin{array}{l}08-26-86 \\
08-26-86\end{array}$ & $-\overline{8.2}$ & 110 & $2 . \overline{5}$ & $\begin{array}{l}225 \\
225\end{array}$ & $\begin{array}{l}0 \\
0\end{array}$ & $\begin{array}{l}184 \\
184\end{array}$ \\
\hline 29 & $08-19-86$ & -- & -- & -- & -- & -- & -- \\
\hline 30 & $08-19-86$ & .5 & 31 & 2.6 & 76 & 4 & 68 \\
\hline 31 & $09-04-86$ & 23 & 61 & 4.7 & 320 & 0 & 262 \\
\hline 32 & $09-05-86$ & 19 & 65 & 3.3 & 412 & 0 & 338 \\
\hline 33 & $11-26-85$ & 5.6 & 15 & 2.4 & 100 & 0 & 87 \\
\hline
\end{tabular}


TABLE 5.--Data on physical properties and major chemical constituents--Continued

\begin{tabular}{|c|c|c|c|c|c|c|c|c|}
\hline $\begin{array}{c}\text { SITE } \\
\text { NUMBER } \\
\text { (figure 2) }\end{array}$ & DATE & $\begin{array}{l}\text { SULFATE, } \\
\text { DIS- } \\
\text { SOLVED } \\
(\mathrm{MG} / \mathrm{L} \\
\left.\text { AS } \mathrm{SO}_{4}\right)\end{array}$ & $\begin{array}{l}\text { CHLO- } \\
\text { RIDE, } \\
\text { DIS- } \\
\text { SOLVED } \\
\text { (MG/L } \\
\text { AS CL) }\end{array}$ & $\begin{array}{l}\text { FLUO- } \\
\text { RIDE, } \\
\text { DIS- } \\
\text { SOLVED } \\
\text { (MG/L } \\
\text { AS F) }\end{array}$ & $\begin{array}{l}\text { SILICA, } \\
\text { DIS- } \\
\text { SOLVED } \\
\text { (MG/L } \\
\text { AS } \\
\mathrm{SIO}_{2} \text { ) }\end{array}$ & $\begin{array}{l}\text { SOLIDS, } \\
\text { RESIDUE } \\
\text { AT } 180 \\
\text { DEG C, } \\
\text { DIS- } \\
\text { SOLVED } \\
\text { (MG/L) }\end{array}$ & $\begin{array}{l}\text { SOLIDS, } \\
\text { SUM OF } \\
\text { CONSTI- } \\
\text { TUENTS, } \\
\text { DIS- } \\
\text { SOLVED } \\
\text { (MG/L) }\end{array}$ & $\begin{array}{l}\text { TUR- } \\
\text { BID- } \\
\text { ITY } \\
\text { (NTU) }\end{array}$ \\
\hline 23 & $\begin{array}{l}11-19-85 \\
02-25-86 \\
05-15-86 \\
08-21-86\end{array}$ & $\begin{array}{l}66 \\
66 \\
65 \\
63\end{array}$ & $\begin{array}{l}22 \\
22 \\
20 \\
16\end{array}$ & $\begin{array}{l}<0.1 \\
<.1 \\
<.1 \\
<.1\end{array}$ & $\begin{array}{l}29 \\
32 \\
32 \\
30\end{array}$ & $\begin{array}{l}348 \\
360 \\
362 \\
330\end{array}$ & $\begin{array}{l}360 \\
360 \\
350 \\
330\end{array}$ & $\begin{array}{r}0.6 \\
.3 \\
1.0 \\
-.\end{array}$ \\
\hline 24 & $\begin{array}{l}11-14-85 \\
02-25-86 \\
05-14-86 \\
08-12-86\end{array}$ & $\begin{array}{l}20 \\
15 \\
19 \\
20\end{array}$ & $\begin{array}{l}1.1 \\
1.3 \\
1.1 \\
1.1\end{array}$ & $\begin{array}{l}.8 \\
.8 \\
.8 \\
.8\end{array}$ & $\begin{array}{l}21 \\
23 \\
21 \\
20\end{array}$ & $\begin{array}{l}82 \\
83 \\
87 \\
83\end{array}$ & $\begin{array}{l}84 \\
82 \\
82 \\
85\end{array}$ & $\begin{array}{r}.8 \\
2.7 \\
1.0 \\
.2\end{array}$ \\
\hline 25 & $\begin{array}{l}11-14-85 \\
02-24-86 \\
05-12-86 \\
08-01-86\end{array}$ & $\begin{array}{l}89 \\
89 \\
88 \\
86\end{array}$ & $\begin{array}{l}4.4 \\
4.5 \\
4.9 \\
4.5\end{array}$ & $\begin{array}{r}.1 \\
.1 \\
<.1 \\
.2\end{array}$ & $\begin{array}{l}39 \\
40 \\
38 \\
39\end{array}$ & $\begin{array}{l}332 \\
338 \\
341 \\
352\end{array}$ & $\begin{array}{l}330 \\
330 \\
320 \\
330\end{array}$ & $\begin{array}{r}.5 \\
1.0 \\
.8\end{array}$ \\
\hline 26 & $\begin{array}{l}11-15-85 \\
02-24-86 \\
05-12-86 \\
08-01-86\end{array}$ & $\begin{array}{l}15 \\
11 \\
12 \\
12\end{array}$ & $\begin{array}{l}18 \\
13 \\
13 \\
11\end{array}$ & $\begin{array}{l}<.1 \\
<.1 \\
<.1 \\
<.1\end{array}$ & $\begin{array}{l}51 \\
51 \\
50 \\
50\end{array}$ & $\begin{array}{l}316 \\
269 \\
271 \\
311\end{array}$ & $\begin{array}{l}310 \\
270 \\
270 \\
280\end{array}$ & $\begin{array}{l}7.1 \\
2.0 \\
1.0 \\
1.0\end{array}$ \\
\hline 27 & $09-02-86$ & 62 & 11 & .4 & 48 & 288 & 300 & - \\
\hline 28 & $\begin{array}{l}08-26-86 \\
08-26-86\end{array}$ & $\overline{120}$ & $\overline{14}$ & -- & $\overline{53}$ & $\overline{440}$ & - & - \\
\hline 29 & $08-19-86$ & - & - & - & - & -- & -- & - \\
\hline 30 & $08-19-86$ & 21 & 4.9 & 1.0 & 55 & 166 & 170 & .2 \\
\hline 31 & $09-04-86$ & 150 & 63 & .2 & 54 & 532 & 620 & - \\
\hline 32 & $09-05-86$ & 46 & 8.3 & .2 & 55 & 476 & 470 & - \\
\hline 33 & $11-26-85$ & 23 & 5.6 & .2 & 33 & 152 & 160 & .5 \\
\hline
\end{tabular}


TABLE 6.--Data on dissolved nitrogen, phosphorus, and organic carbon

[Abbreviations: MG/L, milligrams per liter; "-," data not available; " $<$," less than]

\begin{tabular}{|c|c|c|c|c|c|}
\hline $\begin{array}{l}\text { SITE NUMBER } \\
\text { (f1gure 2) }\end{array}$ & DATE & TIME & $\begin{array}{l}\text { NITRO- } \\
\text { GEN, } \\
\text { DIS- } \\
\text { SOLVED } \\
\text { (MG/L } \\
\text { AS N) }\end{array}$ & $\begin{array}{l}\text { NITRO- } \\
\text { GEN, } \\
\text { AMMONIA, } \\
\text { DIS- } \\
\text { SOLVED } \\
\text { (MG/L } \\
\text { AS N) }\end{array}$ & $\begin{array}{l}\text { NITRO- } \\
\text { GEN, } \\
\text { ORGANIC, } \\
\text { DIS- } \\
\text { SOLVED } \\
\text { (MG/L } \\
\text { AS N) }\end{array}$ \\
\hline 1 & $\begin{array}{l}12-19-85 \\
01-31-86 \\
03-17-86 \\
05-09-86 \\
08-06-86 \\
09-04-86\end{array}$ & $\begin{array}{l}1515 \\
1600 \\
1550 \\
0930 \\
1415 \\
1505\end{array}$ & $\begin{array}{l}1.4 \\
1.4 \\
1.7 \\
1.2 \\
1.3 \\
1.4\end{array}$ & $\begin{array}{l}0.01 \\
<.01 \\
<.01 \\
<.01 \\
.02 \\
<.01\end{array}$ & $\begin{array}{c}<0.2 \\
.2 \\
.2 \\
.3 \\
.3\end{array}$ \\
\hline 2 & $\begin{array}{l}11-18-85 \\
01-27-86 \\
03-17-86 \\
05-14-86\end{array}$ & $\begin{array}{l}1510 \\
1440 \\
1150 \\
1440\end{array}$ & $\begin{array}{l}.5 \\
.6 \\
.6 \\
-\end{array}$ & $\begin{array}{r}.01 \\
.01 \\
<.01 \\
<.01\end{array}$ & $\begin{array}{r}.2 \\
.2 \\
.3 \\
<.2\end{array}$ \\
\hline 3 & $\begin{array}{l}11-18-85 \\
01-28-86 \\
03-17-86 \\
05-16-86 \\
07-31-86 \\
09-04-86\end{array}$ & $\begin{array}{l}1315 \\
1415 \\
1400 \\
1040 \\
1220 \\
1050\end{array}$ & $\begin{array}{l}1.5 \\
1.4 \\
1.4 \\
1.4 \\
1.5 \\
1.4\end{array}$ & $\begin{array}{l}.02 \\
.03 \\
<.01 \\
<.01 \\
<.01 \\
<.01\end{array}$ & $\begin{array}{r}.3 \\
.3 \\
.2 \\
.3 \\
<.2 \\
.3\end{array}$ \\
\hline 4 & $\begin{array}{l}02-28-86 \\
05-15-86 \\
07-30-86 \\
09-03-86\end{array}$ & $\begin{array}{l}0950 \\
1610 \\
1300 \\
1430\end{array}$ & $\begin{array}{l}1.8 \\
3.9 \\
1.5 \\
1.6\end{array}$ & $\begin{array}{r}.03 \\
.27 \\
.02 \\
<.01\end{array}$ & $\begin{array}{l}.4 \\
.2 \\
.3 \\
.5\end{array}$ \\
\hline 5 & $\begin{array}{l}11-19-85 \\
01-29-86 \\
03-19-86 \\
05-15-86 \\
07-30-86 \\
09-03-86\end{array}$ & $\begin{array}{l}1145 \\
1115 \\
1400 \\
1040 \\
1000 \\
1245\end{array}$ & $\begin{array}{l}5.5 \\
5.3 \\
4.9 \\
4.5 \\
4.8 \\
4.4\end{array}$ & $\begin{array}{l}.01 \\
.01 \\
<.01 \\
<.01 \\
<.01 \\
<.01\end{array}$ & $\begin{array}{r}1.1 \\
.8 \\
.5 \\
.2 \\
.4 \\
.4\end{array}$ \\
\hline 6 & $\begin{array}{l}11-15-85 \\
01-27-86 \\
03-19-86 \\
05-12-86 \\
08-04-86 \\
09-03-86\end{array}$ & $\begin{array}{l}1035 \\
0930 \\
1100 \\
1040 \\
1010 \\
1015\end{array}$ & $\begin{array}{r}.8 \\
.9 \\
.5 \\
-. \\
.6 \\
.9\end{array}$ & $\begin{array}{r}.04 \\
.03 \\
<.01 \\
<.01 \\
.02 \\
.01\end{array}$ & $\begin{array}{r}.3 \\
.3 \\
<.2 \\
.2 \\
<.2 \\
.2\end{array}$ \\
\hline
\end{tabular}


TABLE 6.--Data on dissolved nitrogen, phosphorus, and organic carbon--Continued

\begin{tabular}{|c|c|c|c|c|c|c|}
\hline $\begin{array}{l}\text { SITE NUMBER } \\
\text { (figure 2) }\end{array}$ & DATE & $\begin{array}{l}\text { NITRO- } \\
\text { GEN, } \\
\text { NITRITE, } \\
\text { DIS- } \\
\text { SOLVED } \\
\text { (MG/L } \\
\text { AS N) }\end{array}$ & $\begin{array}{l}\text { NITRO- } \\
\text { GEN, } \\
\text { NITRATE, } \\
\text { DIS- } \\
\text { SOLVED } \\
\text { (MG/L } \\
\text { AS N) }\end{array}$ & $\begin{array}{l}\text { PHOS- } \\
\text { PHORUS, } \\
\text { DIS- } \\
\text { SOLVED } \\
\text { (MG/L } \\
\text { AS P) }\end{array}$ & $\begin{array}{l}\text { PHOS- } \\
\text { PHORUS, } \\
\text { ORTHO, } \\
\text { DIS- } \\
\text { SOLVED } \\
\text { (MG/L } \\
\text { AS P) }\end{array}$ & $\begin{array}{l}\text { CARBON, } \\
\text { ORGANIC, } \\
\text { DIS- } \\
\text { SOLVED } \\
\text { (MG/L } \\
\text { AS C) }\end{array}$ \\
\hline 1 & $\begin{array}{l}12-19-85 \\
01-31-86 \\
03-17-86 \\
05-09-86 \\
08-06-86 \\
09-04-86\end{array}$ & $\begin{array}{l}<0.01 \\
<.01 \\
<.01 \\
<.01 \\
<.01 \\
<.01\end{array}$ & $\begin{array}{l}1.4 \\
1.4 \\
1.5 \\
1.0 \\
1.0 \\
1.1\end{array}$ & $\begin{array}{c}<0.01 \\
-- \\
- \\
- \\
- \\
-\end{array}$ & $\begin{array}{c}<0.01 \\
-- \\
- \\
- \\
-\end{array}$ & $\begin{array}{r}1.1 \\
.9 \\
.6 \\
.6 \\
.7 \\
.2\end{array}$ \\
\hline 2 & $\begin{array}{l}11-18-85 \\
01-27-86 \\
03-17-86 \\
05-14-86\end{array}$ & $\begin{array}{r}<.01 \\
.02 \\
<.01 \\
<.01\end{array}$ & $\begin{array}{l}.32 \\
.35 \\
.30 \\
.25\end{array}$ & $\frac{.02}{-}$ & $\begin{array}{l}.02 \\
-- \\
-- \\
--\end{array}$ & $\begin{array}{l}.5 \\
.4 \\
.7 \\
.2\end{array}$ \\
\hline 3 & $\begin{array}{l}11-18-85 \\
01-28-86 \\
03-17-86 \\
05-16-86 \\
07-31-86 \\
09-04-86\end{array}$ & $\begin{array}{l}<.01 \\
.01 \\
<.01 \\
<.01 \\
<.01 \\
<.01\end{array}$ & $\begin{array}{l}1.2 \\
1.1 \\
1.2 \\
1.2 \\
1.5 \\
1.1\end{array}$ & $\begin{array}{l}.02 \\
- \\
- \\
- \\
-\end{array}$ & $\begin{array}{l}.02 \\
-- \\
-- \\
- \\
-- \\
-\end{array}$ & $\begin{array}{r}1.4 \\
.8 \\
.8 \\
.3 \\
.6 \\
.3\end{array}$ \\
\hline 4 & $\begin{array}{l}02-28-86 \\
05-15-86 \\
07-30-86 \\
09-03-86\end{array}$ & $\begin{array}{r}<.01 \\
.01 \\
<.01 \\
<.01\end{array}$ & $\begin{array}{l}1.4 \\
3.4 \\
1.2 \\
1.1\end{array}$ & - & - & $\begin{array}{l}.7 \\
.7 \\
.5 \\
.3\end{array}$ \\
\hline 5 & $\begin{array}{l}11-19-85 \\
01-29-86 \\
03-19-86 \\
05-15-86 \\
07-30-86 \\
09-03-86\end{array}$ & $\begin{array}{l}<.01 \\
.01 \\
<.01 \\
<.01 \\
<.01 \\
<.01\end{array}$ & $\begin{array}{l}4.4 \\
4.5 \\
4.4 \\
4.3 \\
4.4 \\
4.0\end{array}$ & $\begin{array}{l}.14 \\
- \\
- \\
-\end{array}$ & $\begin{array}{l}.02 \\
- \\
- \\
- \\
-\end{array}$ & $\begin{array}{l}.6 \\
.5 \\
.5 \\
.5 \\
.6 \\
.5\end{array}$ \\
\hline 6 & $\begin{array}{l}11-15-85 \\
01-27-86 \\
03-19-86 \\
05-12-86 \\
08-04-86 \\
09-03-86\end{array}$ & $\begin{array}{l}<.01 \\
.02 \\
<.01 \\
<.01 \\
<.01 \\
<.01\end{array}$ & $\begin{array}{r}.53 \\
.54 \\
.48 \\
<.10 \\
.60 \\
.69\end{array}$ & $\begin{array}{l}.04 \\
- \\
- \\
-\end{array}$ & $\begin{array}{l}.05 \\
- \\
-- \\
-- \\
--\end{array}$ & $\begin{array}{l}.6 \\
.7 \\
.6 \\
.7 \\
.8 \\
.2\end{array}$ \\
\hline
\end{tabular}


TABLE 6.--Data on dissolved nitrogen, phosphorus, and organic carbon--Continued

\begin{tabular}{|c|c|c|c|c|c|}
\hline $\begin{array}{l}\text { SITE NUMBER } \\
\text { (figure 2) }\end{array}$ & DATE & TIME & $\begin{array}{l}\text { NITRO- } \\
\text { GEN, } \\
\text { DIS- } \\
\text { SOLVED } \\
\text { (MG/L } \\
\text { AS N) }\end{array}$ & $\begin{array}{l}\text { NITRO- } \\
\text { GEN, } \\
\text { AMMONIA, } \\
\text { DIS- } \\
\text { SOLVED } \\
\text { (MG/L } \\
\text { AS N) }\end{array}$ & $\begin{array}{l}\text { NITRO- } \\
\text { GEN, } \\
\text { ORGANIC, } \\
\text { DIS- } \\
\text { SOLVED } \\
\text { (MG/L } \\
\text { AS N) }\end{array}$ \\
\hline 7 & $\begin{array}{l}12-03-85 \\
02-27-86 \\
05-09-86 \\
08-11-86\end{array}$ & $\begin{array}{l}1415 \\
0945 \\
1240 \\
1010\end{array}$ & $\begin{array}{l}20 \\
21 \\
21 \\
20\end{array}$ & $\begin{array}{r}0.02 \\
.03 \\
<.01 \\
<.03\end{array}$ & $\begin{array}{r}0.3 \\
.2 \\
.4 \\
.8\end{array}$ \\
\hline 8 & $\begin{array}{l}12-03-85 \\
02-26-86 \\
05-09-86 \\
08-19-86\end{array}$ & $\begin{array}{l}1215 \\
1505 \\
1450 \\
1240\end{array}$ & $\begin{array}{l}.2 \\
.6 \\
.6 \\
.4\end{array}$ & $\begin{array}{l}<.01 \\
.02 \\
.01 \\
.02\end{array}$ & $\begin{array}{r}<.2 \\
.4 \\
.2 \\
<.2\end{array}$ \\
\hline 9 & $\begin{array}{l}11-22-85 \\
02-27-86 \\
05-09-86 \\
08-12-86\end{array}$ & $\begin{array}{l}0950 \\
1230 \\
1600 \\
1440\end{array}$ & $\begin{array}{l}1.0 \\
1.3 \\
1.1 \\
1.2\end{array}$ & $\begin{array}{r}.02 \\
<.01 \\
<.01 \\
.01\end{array}$ & $\begin{array}{r}<.2 \\
.3 \\
.2 \\
.2\end{array}$ \\
\hline 10 & $\begin{array}{l}12-09-85 \\
02-10-86 \\
05-19-86 \\
08-13-86\end{array}$ & $\begin{array}{l}1210 \\
1200 \\
1150 \\
1020\end{array}$ & $\begin{array}{l}.4 \\
.4 \\
.3 \\
.3\end{array}$ & $\begin{array}{l}.21 \\
.16 \\
.19 \\
.20\end{array}$ & $\begin{array}{l}.2 \\
.2 \\
.1 \\
.1\end{array}$ \\
\hline 11 & $\begin{array}{l}11-20-85 \\
02-26-86 \\
05-20-86 \\
08-13-86\end{array}$ & $\begin{array}{l}1445 \\
1215 \\
1430 \\
1200\end{array}$ & $\begin{array}{r}.5 \\
1.1 \\
.8 \\
.6\end{array}$ & $\begin{array}{l}.02 \\
<.01 \\
<.01 \\
.02\end{array}$ & $\begin{array}{r}<.2 \\
.5 \\
.2 \\
<.2\end{array}$ \\
\hline 12 & $\begin{array}{l}11-22-85 \\
02-12-86 \\
05-20-86 \\
08-13-86\end{array}$ & $\begin{array}{l}1245 \\
1400 \\
1230 \\
1400\end{array}$ & $\begin{array}{l}1.3 \\
1.3 \\
1.1 \\
1.0\end{array}$ & $\begin{array}{r}.03 \\
.01 \\
<.01 \\
<.01\end{array}$ & $\begin{array}{r}.4 \\
.4 \\
.2 \\
<.2\end{array}$ \\
\hline 13 & $\begin{array}{l}11-22-85 \\
02-12-86 \\
05-20-86 \\
08-27-86\end{array}$ & $\begin{array}{l}1515 \\
1200 \\
1000 \\
1040\end{array}$ & $\begin{array}{l}1.7 \\
1.7 \\
1.8 \\
2.2\end{array}$ & $\begin{array}{r}.02 \\
.02 \\
<.01 \\
.03\end{array}$ & $\begin{array}{l}.3 \\
.3 \\
.3 \\
.7\end{array}$ \\
\hline 14 & $\begin{array}{l}11-25-85 \\
02-06-86 \\
05-13-86 \\
08-18-86\end{array}$ & $\begin{array}{l}1335 \\
1030 \\
1200 \\
1345\end{array}$ & $\begin{array}{l}.2 \\
.6 \\
.2 \\
.2\end{array}$ & $\begin{array}{r}.02 \\
.03 \\
<.01 \\
.01\end{array}$ & $\begin{array}{r}<.2 \\
.4 \\
<.2 \\
<.2\end{array}$ \\
\hline
\end{tabular}


TABLE 6.--Data on dissolved nitrogen, phosphorus, and organic carbon--Continued

\begin{tabular}{|c|c|c|c|c|c|c|}
\hline $\begin{array}{l}\text { SITE NUMBER } \\
\text { (f1gure 2) }\end{array}$ & DATE & $\begin{array}{l}\text { NITRO- } \\
\text { GEN, } \\
\text { NITRITE, } \\
\text { DIS- } \\
\text { SOLVED } \\
\text { (MG/L } \\
\text { AS N) }\end{array}$ & $\begin{array}{l}\text { NITRO- } \\
\text { GEN, } \\
\text { NITRATE, } \\
\text { DIS- } \\
\text { SOLVED } \\
\text { (MG/L } \\
\text { AS N) }\end{array}$ & $\begin{array}{l}\text { PHOS- } \\
\text { PHORUS, } \\
\text { DIS- } \\
\text { SOLVED } \\
\text { (MG/L } \\
\text { AS P) }\end{array}$ & $\begin{array}{l}\text { PHOS- } \\
\text { PHORUS, } \\
\text { ORTHO, } \\
\text { DIS- } \\
\text { SOLVED } \\
\text { (MG/L } \\
\text { AS P) }\end{array}$ & $\begin{array}{l}\text { CARBON, } \\
\text { ORGANIC, } \\
\text { DIS- } \\
\text { SOLVED } \\
\text { (MG/L } \\
\text { AS C) }\end{array}$ \\
\hline 7 & $\begin{array}{l}12-03-85 \\
02-27-86 \\
05-09-86 \\
08-11-86\end{array}$ & $\begin{array}{l}<0.01 \\
<.01 \\
<.01 \\
<.01\end{array}$ & $\begin{array}{l}20 \\
21 \\
21 \\
19\end{array}$ & $\begin{array}{c}0.05 \\
- \\
- \\
-\end{array}$ & $\begin{array}{c}0.05 \\
-- \\
- \\
-\end{array}$ & $\begin{array}{l}2.6 \\
2.2 \\
2.4 \\
2.4\end{array}$ \\
\hline 8 & $\begin{array}{l}12-03-85 \\
02-26-86 \\
05-09-86 \\
08-19-86\end{array}$ & $\begin{array}{l}.01 \\
<.01 \\
<.01 \\
<.01\end{array}$ & $\begin{array}{l}.21 \\
.24 \\
.40 \\
.43\end{array}$ & $\frac{.05}{-}$ & $\begin{array}{l}.05 \\
- \\
-\end{array}$ & $\begin{array}{l}.5 \\
.4 \\
.7 \\
.7\end{array}$ \\
\hline 9 & $\begin{array}{l}11-22-85 \\
02-27-86 \\
05-09-86 \\
08-12-86\end{array}$ & $\begin{array}{l}<.01 \\
<.01 \\
<.01 \\
<.01\end{array}$ & $\begin{array}{l}1.0 \\
1.0 \\
.93 \\
1.0\end{array}$ & $\begin{array}{l}.06 \\
- \\
- \\
-\end{array}$ & $\begin{array}{l}.01 \\
- \\
-- \\
--\end{array}$ & $\begin{array}{l}.9 \\
.5 \\
.7 \\
.5\end{array}$ \\
\hline 10 & $\begin{array}{l}12-09-85 \\
02-10-86 \\
05-19-86 \\
08-13-86\end{array}$ & $\begin{array}{l}<.01 \\
<.01 \\
<.01 \\
<.01\end{array}$ & $\begin{array}{l}<.10 \\
<.10 \\
<.10 \\
<.10\end{array}$ & $\frac{.14}{-}$ & $\begin{array}{l}.06 \\
- \\
-- \\
-\end{array}$ & $\begin{array}{l}2.4 \\
2.2 \\
2.5 \\
3.0\end{array}$ \\
\hline 11 & $\begin{array}{l}11-20-85 \\
02-26-86 \\
05-20-86 \\
08-13-86\end{array}$ & $\begin{array}{l}<.01 \\
<.01 \\
<.01 \\
<.01\end{array}$ & $\begin{array}{l}.54 \\
.64 \\
.58 \\
.62\end{array}$ & $\frac{.02}{-}$ & $\begin{array}{l}.03 \\
-- \\
--\end{array}$ & $\begin{array}{l}.4 \\
.6 \\
.3 \\
.6\end{array}$ \\
\hline 12 & $\begin{array}{l}11-22-85 \\
02-12-86 \\
05-20-86 \\
08-13-86\end{array}$ & $\begin{array}{l}.01 \\
<.01 \\
<.01 \\
<.01\end{array}$ & $\begin{array}{l}.90 \\
.90 \\
.90 \\
.97\end{array}$ & $\begin{array}{l}.24 \\
-- \\
-\end{array}$ & $\begin{array}{l}.04 \\
- \\
- \\
-\end{array}$ & $\begin{array}{l}.5 \\
.4 \\
.3 \\
.4\end{array}$ \\
\hline 13 & $\begin{array}{l}11-22-85 \\
02-12-86 \\
05-20-86 \\
08-27-86\end{array}$ & $\begin{array}{l}.01 \\
<.01 \\
<.01 \\
<.01\end{array}$ & $\begin{array}{l}1.4 \\
1.4 \\
1.5 \\
1.5\end{array}$ & $\frac{.26}{-}$ & $\begin{array}{l}.02 \\
-- \\
-- \\
--\end{array}$ & $\begin{array}{l}.8 \\
.5 \\
.2 \\
.4\end{array}$ \\
\hline 14 & $\begin{array}{l}11-25-85 \\
02-06-86 \\
05-13-86 \\
08-18-86\end{array}$ & $\begin{array}{l}.01 \\
<.01 \\
<.01 \\
<.01\end{array}$ & $\begin{array}{l}.21 \\
.21 \\
.21 \\
.19\end{array}$ & $\frac{.09}{-}$ & $\begin{array}{l}.02 \\
- \\
-\end{array}$ & $\begin{array}{l}.7 \\
.7 \\
.6 \\
.8\end{array}$ \\
\hline
\end{tabular}


TABLE 6.--Data on dissolved nitrogen, phosphorus, and organic carbon--Continued

\begin{tabular}{|c|c|c|c|c|c|}
\hline $\begin{array}{l}\text { SITE NUMBER } \\
\text { (f 1gure 2) }\end{array}$ & DATE & TIME & $\begin{array}{l}\text { NITRO- } \\
\text { GEN, } \\
\text { DIS- } \\
\text { SOLVED } \\
\text { (MG/L } \\
\text { AS N) }\end{array}$ & $\begin{array}{l}\text { NITRO- } \\
\text { GEN, } \\
\text { AMMONIA, } \\
\text { DIS- } \\
\text { SOLVED } \\
\text { (MG/L } \\
\text { AS N) }\end{array}$ & $\begin{array}{l}\text { NITRO- } \\
\text { GEN, } \\
\text { ORGANIC, } \\
\text { DIS- } \\
\text { SOLVED } \\
(M G / L \\
\text { AS N) }\end{array}$ \\
\hline 15 & $\begin{array}{l}11-20-85 \\
02-10-86 \\
05-13-86\end{array}$ & $\begin{array}{l}1235 \\
1430 \\
1610\end{array}$ & $\begin{array}{r}0.2 \\
.3 \\
.2\end{array}$ & $\begin{array}{r}0.04 \\
.05 \\
.03\end{array}$ & $\begin{array}{r}0.2 \\
.2 \\
.2\end{array}$ \\
\hline 16 & $\begin{array}{l}11-27-85 \\
02-06-86 \\
05-13-86 \\
08-18-86\end{array}$ & $\begin{array}{l}1245 \\
1235 \\
1350 \\
1140\end{array}$ & $\begin{array}{l}1.8 \\
2.0 \\
1.7 \\
1.7\end{array}$ & $\begin{array}{r}.02 \\
.02 \\
<.01 \\
.02\end{array}$ & $\begin{array}{l}.3 \\
.5 \\
.2 \\
.3\end{array}$ \\
\hline 17 & $\begin{array}{l}11-25-85 \\
02-06-86 \\
05-13-86 \\
08-18-86\end{array}$ & $\begin{array}{l}1030 \\
1435 \\
1010 \\
1000\end{array}$ & $\begin{array}{l}.3 \\
.6 \\
.6 \\
.5\end{array}$ & $\begin{array}{l}.14 \\
.12 \\
.09 \\
.14\end{array}$ & $\begin{array}{l}.1 \\
.2 \\
.1 \\
.1\end{array}$ \\
\hline 18 & $\begin{array}{l}11-26-85 \\
02-25-86 \\
05-19-86 \\
08-26-86\end{array}$ & $\begin{array}{l}1605 \\
1530 \\
1510 \\
1050\end{array}$ & $\begin{array}{r}.9 \\
.7 \\
1.0 \\
.7\end{array}$ & $\begin{array}{l}.02 \\
.02 \\
.01 \\
.02\end{array}$ & $\begin{array}{r}.3 \\
.2 \\
.3 \\
<.2\end{array}$ \\
\hline 19 & $\begin{array}{l}12-10-85 \\
02-11-86 \\
05-14-86 \\
08-12-86\end{array}$ & $\begin{array}{l}1305 \\
1250 \\
1240 \\
1250\end{array}$ & $\begin{array}{l}2.3 \\
2.5 \\
2.7 \\
2.8\end{array}$ & $\begin{array}{r}.01 \\
.01 \\
<.01 \\
.02\end{array}$ & $\begin{array}{l}.2 \\
.5 \\
.6 \\
.8\end{array}$ \\
\hline 20 & $\begin{array}{l}11-13-85 \\
02-07-86 \\
05-16-86 \\
08-21-86\end{array}$ & $\begin{array}{l}1400 \\
1315 \\
1240 \\
1535\end{array}$ & $\begin{array}{l}.7 \\
.9 \\
.5 \\
.8\end{array}$ & $\begin{array}{r}.01 \\
.04 \\
<.01 \\
<.01\end{array}$ & $\begin{array}{r}.2 \\
.4 \\
<.2 \\
.2\end{array}$ \\
\hline 21 & $\begin{array}{l}11-20-85 \\
02-07-86 \\
05-15-86 \\
08-21-86\end{array}$ & $\begin{array}{l}1030 \\
1040 \\
1410 \\
1340\end{array}$ & $\begin{array}{l}1.4 \\
1.4 \\
1.2 \\
1.2\end{array}$ & $\begin{array}{r}.01 \\
.02 \\
<.01 \\
.01\end{array}$ & $\begin{array}{l}.4 \\
.3 \\
.2 \\
.2\end{array}$ \\
\hline 22 & $\begin{array}{l}11-26-85 \\
02-11-86 \\
05-14-86 \\
08-21-86\end{array}$ & $\begin{array}{l}1325 \\
1500 \\
1630 \\
1000\end{array}$ & $\begin{array}{l}1.5 \\
1.6 \\
1.6 \\
1.5\end{array}$ & $\begin{array}{r}.03 \\
.03 \\
.01 \\
<.01\end{array}$ & $\begin{array}{l}.3 \\
.6 \\
.2 \\
.2\end{array}$ \\
\hline
\end{tabular}


TABLE 6.--Data on dissolved nitrogen, phosphorus, and organic carbon--Continued

\begin{tabular}{|c|c|c|c|c|c|c|}
\hline $\begin{array}{l}\text { SITE NUMBER } \\
\text { (f1gure 2) }\end{array}$ & DATE & $\begin{array}{l}\text { NITRO- } \\
\text { GEN, } \\
\text { NITRITE, } \\
\text { DIS- } \\
\text { SOLVED } \\
\text { (MG/L } \\
\text { AS N) }\end{array}$ & $\begin{array}{l}\text { NITRO- } \\
\text { GEN, } \\
\text { NITRATE, } \\
\text { DIS- } \\
\text { SOLVED } \\
\text { (MG/L } \\
\text { AS N) }\end{array}$ & $\begin{array}{l}\text { PHOS- } \\
\text { PHORUS, } \\
\text { DIS- } \\
\text { SOLVED } \\
\text { (MG/L } \\
\text { AS P) }\end{array}$ & $\begin{array}{l}\text { PHOS- } \\
\text { PHORUS, } \\
\text { ORTHO, } \\
\text { DIS- } \\
\text { SOLVED } \\
\text { (MG/L } \\
\text { AS P) }\end{array}$ & $\begin{array}{l}\text { CARBON, } \\
\text { ORGANIC, } \\
\text { DIS- } \\
\text { SOLVED } \\
\text { (MG/L } \\
\text { AS C) }\end{array}$ \\
\hline 15 & $\begin{array}{l}11-20-85 \\
02-10-86 \\
05-13-86\end{array}$ & $\begin{array}{l}<0.01 \\
<.01 \\
<.01\end{array}$ & $\begin{array}{r}<0.10 \\
<.10 \\
<.10\end{array}$ & $\frac{0.02}{-}$ & $\begin{array}{c}0.03 \\
-- \\
-\end{array}$ & $\begin{array}{r}0.8 \\
.6 \\
.6\end{array}$ \\
\hline 16 & $\begin{array}{l}11-27-85 \\
02-06-86 \\
05-13-86 \\
08-18-86\end{array}$ & $\begin{array}{l}<.01 \\
<.01 \\
<.01 \\
<.01\end{array}$ & $\begin{array}{l}1.5 \\
1.5 \\
1.5 \\
1.4\end{array}$ & $\begin{array}{l}.07 \\
-- \\
-\end{array}$ & $\begin{array}{l}.06 \\
-- \\
--\end{array}$ & $\begin{array}{l}.8 \\
.5 \\
.7 \\
.4\end{array}$ \\
\hline 17 & $\begin{array}{l}11-25-85 \\
02-06-86 \\
05-13-86 \\
08-18-86\end{array}$ & $\begin{array}{l}.01 \\
.03 \\
.03 \\
.03\end{array}$ & $\begin{array}{l}.13 \\
.29 \\
.35 \\
.25\end{array}$ & $\begin{array}{l}.27 \\
-- \\
-- \\
-\end{array}$ & $\begin{array}{l}.12 \\
-- \\
-- \\
--\end{array}$ & $\begin{array}{r}.8 \\
1.1 \\
.9 \\
.5\end{array}$ \\
\hline 18 & $\begin{array}{l}11-26-85 \\
02-25-86 \\
05-19-86 \\
08-26-86\end{array}$ & $\begin{array}{l}<.01 \\
<.01 \\
<.01 \\
<.01\end{array}$ & $\begin{array}{l}.61 \\
.54 \\
.67 \\
.71\end{array}$ & $\begin{array}{l}.06 \\
- \\
--\end{array}$ & $\begin{array}{l}.06 \\
- \\
-\end{array}$ & $\begin{array}{l}.7 \\
.7 \\
.4 \\
.5\end{array}$ \\
\hline 19 & $\begin{array}{l}12-10-85 \\
02-11-86 \\
05-14-86 \\
08-12-86\end{array}$ & $\begin{array}{l}<.01 \\
<.01 \\
<.01 \\
<.01\end{array}$ & $\begin{array}{l}2.1 \\
2.0 \\
2.1 \\
1.0\end{array}$ & $\frac{.03}{-}$ & $\begin{array}{l}.04 \\
- \\
-\end{array}$ & $\begin{array}{r}.8 \\
.5 \\
.3 \\
1.2\end{array}$ \\
\hline 20 & $\begin{array}{l}11-13-85 \\
02-07-86 \\
05-16-86 \\
08-21-86\end{array}$ & $\begin{array}{l}<.01 \\
<.01 \\
<.01 \\
<.01\end{array}$ & $\begin{array}{l}.52 \\
.50 \\
.49 \\
.59\end{array}$ & $\begin{array}{l}.15 \\
-\infty \\
-\infty\end{array}$ & $\begin{array}{l}.14 \\
-- \\
--\end{array}$ & $\begin{array}{r}.7 \\
.7 \\
.5 \\
1.0\end{array}$ \\
\hline 21 & $\begin{array}{l}11-20-85 \\
02-07-86 \\
05-15-86 \\
08-21-86\end{array}$ & $\begin{array}{l}<.01 \\
<.01 \\
<.01 \\
<.01\end{array}$ & $\begin{array}{l}.98 \\
1.1 \\
1.0 \\
1.0\end{array}$ & $\begin{array}{l}.07 \\
-- \\
- \\
-\end{array}$ & $\frac{.07}{-}$ & $\begin{array}{r}.5 \\
1.0 \\
.3 \\
.4\end{array}$ \\
\hline 22 & $\begin{array}{l}11-26-85 \\
02-11-86 \\
05-14-86 \\
08-21-86\end{array}$ & $\begin{array}{l}<.01 \\
<.01 \\
<.01 \\
<.01\end{array}$ & $\begin{array}{l}1.2 \\
1.0 \\
1.4 \\
1.3\end{array}$ & $\begin{array}{l}.09 \\
- \\
-\end{array}$ & $\begin{array}{l}.09 \\
-- \\
- \\
-\end{array}$ & $\begin{array}{r}1.2 \\
1.0 \\
.8 \\
.8\end{array}$ \\
\hline
\end{tabular}


TABLE 6.--Data on dissolved nitrogen, phosphorus, and organic carbon--Continued

\begin{tabular}{|c|c|c|c|c|c|}
\hline $\begin{array}{l}\text { SITE NUMBER } \\
\text { (figure 2) }\end{array}$ & DATE & TIME & $\begin{array}{l}\text { NITRO- } \\
\text { GEN, } \\
\text { DIS- } \\
\text { SOLVED } \\
\text { (MG/L } \\
\text { AS N) }\end{array}$ & $\begin{array}{l}\text { NITRO- } \\
\text { GEN, } \\
\text { AMMONIA, } \\
\text { DIS } \\
\text { SOLVED } \\
\text { (MG/L } \\
\text { AS N) }\end{array}$ & $\begin{array}{l}\text { NITRO- } \\
\text { GEN, } \\
\text { ORGANIC, } \\
\text { DIS- } \\
\text { SOLVED } \\
\text { (MG/L } \\
\text { AS N) }\end{array}$ \\
\hline 23 & $\begin{array}{l}11-19-85 \\
02-25-86 \\
05-15-86 \\
08-21-86\end{array}$ & $\begin{array}{l}1420 \\
1240 \\
1200 \\
1210\end{array}$ & $\begin{array}{l}3.3 \\
3.6 \\
3.2 \\
3.3\end{array}$ & $\begin{array}{r}0.03 \\
.01 \\
.02 \\
.02\end{array}$ & $\begin{array}{r}0.3 \\
.5 \\
.3 \\
.4\end{array}$ \\
\hline 24 & $\begin{array}{l}11-14-85 \\
02-25-86 \\
05-14-86 \\
08-12-86\end{array}$ & $\begin{array}{l}1510 \\
1015 \\
1745 \\
1000\end{array}$ & $\begin{array}{l}.3 \\
.3 \\
.3 \\
.2\end{array}$ & $\begin{array}{l}.12 \\
.13 \\
.08 \\
.05\end{array}$ & $\begin{array}{l}.2 \\
.2 \\
.2 \\
.2\end{array}$ \\
\hline 25 & $\begin{array}{l}11-14-85 \\
02-24-86 \\
05-12-86 \\
08-01-86\end{array}$ & $\begin{array}{l}1215 \\
1200 \\
1235 \\
1100\end{array}$ & $\begin{array}{l}3.4 \\
3.3 \\
3.2 \\
3.5\end{array}$ & $\begin{array}{r}.02 \\
.02 \\
<.01 \\
.01\end{array}$ & $\begin{array}{l}.5 \\
.3 \\
.2 \\
.5\end{array}$ \\
\hline 26 & $\begin{array}{l}11-15-85 \\
02-24-86 \\
05-12-86 \\
08-01-86\end{array}$ & $\begin{array}{l}1335 \\
1400 \\
1430 \\
1310\end{array}$ & $\begin{array}{l}6.6 \\
4.3 \\
4.5 \\
5.5\end{array}$ & $\begin{array}{r}.01 \\
.01 \\
<.01 \\
.02\end{array}$ & $\begin{array}{l}.7 \\
.3 \\
.2 \\
.4\end{array}$ \\
\hline 27 & $09-02-86$ & 1545 & .4 & .22 & .2 \\
\hline 28 & $08-26-86$ & 1400 & .6 & .44 & .2 \\
\hline 30 & $08-19-86$ & 1430 & $<.2$ & .02 & $<.2$ \\
\hline 31 & $09-04-86$ & 1340 & 2.3 & 1.2 & .4 \\
\hline 32 & $09-05-86$ & 1445 & 2.1 & .02 & 1.1 \\
\hline 33 & $11-26-85$ & 1055 & .5 & .02 & .2 \\
\hline
\end{tabular}


TABLE 6.--Data on dissolved nitrogen, phosphorus, and organic carbon--Continued

\begin{tabular}{|c|c|c|c|c|c|c|}
\hline $\begin{array}{l}\text { SITE NUMBER } \\
\text { (figure 2) }\end{array}$ & DATE & $\begin{array}{l}\text { NITRO- } \\
\text { GEN, } \\
\text { NITRITE, } \\
\text { DIS- } \\
\text { SOLVED } \\
\text { (MG/L } \\
\text { AS N) }\end{array}$ & $\begin{array}{l}\text { NITRO- } \\
\text { GEN, } \\
\text { NITRATE, } \\
\text { DIS- } \\
\text { SOLVED } \\
\text { (MG/L } \\
\text { AS N) }\end{array}$ & $\begin{array}{l}\text { PHOS- } \\
\text { PHORUS, } \\
\text { DIS- } \\
\text { SOLVED } \\
\text { (MG/L } \\
\text { AS P) }\end{array}$ & $\begin{array}{l}\text { PHOS- } \\
\text { PHORUS, } \\
\text { ORTHO, } \\
\text { DIS- } \\
\text { SOLVED } \\
\text { (MG/L } \\
\text { AS P) }\end{array}$ & $\begin{array}{l}\text { CARBON, } \\
\text { ORGANIC, } \\
\text { DIS- } \\
\text { SOLVED } \\
\text { (MG/L } \\
\text { AS C) }\end{array}$ \\
\hline 23 & $\begin{array}{l}11-19-85 \\
02-25-86 \\
05-15-86 \\
08-21-86\end{array}$ & $\begin{array}{l}0.01 \\
<.01 \\
<.01 \\
<.01\end{array}$ & $\begin{array}{l}3.0 \\
3.1 \\
2.9 \\
2.9\end{array}$ & $\begin{array}{c}0.05 \\
- \\
- \\
-\end{array}$ & $\begin{array}{c}0.05 \\
- \\
- \\
-\end{array}$ & $\begin{array}{r}0.8 \\
1.1 \\
.8 \\
1.0\end{array}$ \\
\hline 24 & $\begin{array}{l}11-14-85 \\
02-25-86 \\
05-14-86 \\
08-12-86\end{array}$ & $\begin{array}{l}<.01 \\
<.01 \\
<.01 \\
<.01\end{array}$ & $\begin{array}{l}<.10 \\
<.10 \\
<.10 \\
<.10\end{array}$ & $\frac{.02}{-}$ & $\begin{array}{l}.01 \\
- \\
- \\
-\end{array}$ & $\begin{array}{l}.6 \\
.6 \\
.3 \\
.5\end{array}$ \\
\hline 25 & $\begin{array}{l}11-14-85 \\
02-24-86 \\
05-12-86 \\
08-01-86\end{array}$ & $\begin{array}{l}<.01 \\
<.01 \\
<.01 \\
<.01\end{array}$ & $\begin{array}{l}2.9 \\
3.0 \\
3.0 \\
3.0\end{array}$ & $\begin{array}{l}.13 \\
-- \\
--\end{array}$ & $\frac{.12}{-}$ & $\begin{array}{l}.4 \\
.6 \\
.5 \\
.5\end{array}$ \\
\hline 26 & $\begin{array}{l}11-15-85 \\
02-24-86 \\
05-12-86 \\
08-01-86\end{array}$ & $\begin{array}{l}<.01 \\
<.01 \\
<.01 \\
<.01\end{array}$ & $\begin{array}{l}5.9 \\
4.0 \\
4.3 \\
5.1\end{array}$ & $\begin{array}{l}.08 \\
- \\
-\end{array}$ & $\frac{.08}{-}$ & $\begin{array}{l}.5 \\
.7 \\
.7 \\
.5\end{array}$ \\
\hline 27 & $09-02-86$ & $<.01$ & $<.10$ & - & -- & 1.5 \\
\hline 28 & $08-26-86$ & $<.01$ & $<.10$ & - & -- & 4.4 \\
\hline 30 & $08-19-86$ & $<.01$ & $<.10$ & - & - & .3 \\
\hline 31 & $09-04-86$ & .05 & .62 & - & - & 6.3 \\
\hline 32 & $09-05-86$ & .06 & .93 & - & - & 3.4 \\
\hline 33 & $11-26-85$ & $<.01$ & .28 & .03 & .03 & .9 \\
\hline
\end{tabular}


TABLE 7.--Data on trace elements

[Abbreviations: UG/L, micrograms per 11ter; "--," data not avallable; $"<, "$ less than]

\begin{tabular}{|c|c|c|c|c|c|c|c|c|}
\hline $\begin{array}{l}\text { SITE NUMBER } \\
\text { (f1gure 2) }\end{array}$ & DATE & TIME & $\begin{array}{l}\text { ARSENIC, } \\
\text { DIS- } \\
\text { SOLVED } \\
\text { (UG/L } \\
\text { AS AS) }\end{array}$ & $\begin{array}{l}\text { BARIUM, } \\
\text { DIS- } \\
\text { SOLVED } \\
\text { (UG/L } \\
\text { AS BA) }\end{array}$ & $\begin{array}{l}\text { BORON, } \\
\text { DIS- } \\
\text { SOLVED } \\
\text { (UG/L } \\
\text { AS B) }\end{array}$ & $\begin{array}{l}\text { CADMIUM, } \\
\text { DIS- } \\
\text { SOLVED } \\
\text { (UG/L } \\
\text { AS CD) }\end{array}$ & $\begin{array}{l}\text { CHRO- } \\
\text { MIUM, } \\
\text { DIS- } \\
\text { SOLVED } \\
\text { (UG/L } \\
\text { AS CK) }\end{array}$ & $\begin{array}{l}\text { CUPPER, } \\
\text { DIS- } \\
\text { SOLVED } \\
\text { (UG/L } \\
\text { AS CU) }\end{array}$ \\
\hline 1 & $\begin{array}{l}12-19-85 \\
01-31-86 \\
03-17-86 \\
05-09-86 \\
08-06-86 \\
09-04-86\end{array}$ & $\begin{array}{l}1515 \\
1600 \\
1550 \\
0930 \\
1415 \\
1505\end{array}$ & $\begin{array}{r}5 \\
5 \\
5 \\
12 \\
12 \\
12\end{array}$ & $\begin{array}{l}40 \\
41 \\
49 \\
28 \\
14 \\
16\end{array}$ & $\begin{array}{l}120 \\
- \\
110 \\
120 \\
130 \\
120\end{array}$ & $\begin{array}{l}<1 \\
<1 \\
<1 \\
<1 \\
<1 \\
<1\end{array}$ & $\begin{array}{r}<10 \\
20 \\
<10 \\
<10 \\
<10 \\
<10\end{array}$ & $\begin{array}{r}10 \\
<10 \\
<10 \\
<10 \\
<10 \\
<10\end{array}$ \\
\hline 2 & $\begin{array}{l}11-18-85 \\
01-27-86 \\
03-17-86 \\
05-14-86\end{array}$ & $\begin{array}{l}1510 \\
1440 \\
1150 \\
1440\end{array}$ & $\begin{array}{r}10 \\
9 \\
10 \\
11\end{array}$ & $\begin{array}{l}66 \\
71 \\
63 \\
60\end{array}$ & $\begin{array}{l}170 \\
160 \\
160\end{array}$ & $\begin{array}{l}<1 \\
<1 \\
<1 \\
<1\end{array}$ & $\begin{array}{l}<10 \\
<10 \\
<10 \\
<10\end{array}$ & $\begin{array}{l}<10 \\
<10 \\
<10 \\
<10\end{array}$ \\
\hline 3 & $\begin{array}{l}11-18-85 \\
01-28-86 \\
03-17-86 \\
05-16-86 \\
07-31-86 \\
09-04-86\end{array}$ & $\begin{array}{l}1315 \\
1415 \\
1400 \\
1040 \\
1220 \\
1050\end{array}$ & $\begin{array}{l}2 \\
2 \\
2 \\
2 \\
1 \\
2\end{array}$ & $\begin{array}{l}140 \\
130 \\
140 \\
130 \\
150 \\
130\end{array}$ & $\begin{array}{l}220 \\
- \\
210 \\
210 \\
230 \\
210\end{array}$ & $\begin{array}{l}2 \\
<1 \\
<1 \\
<1 \\
<1 \\
<1\end{array}$ & $\begin{array}{r}<10 \\
10 \\
<10 \\
<10 \\
<10 \\
<10\end{array}$ & $\begin{array}{l}<10 \\
<10 \\
<10 \\
<10 \\
<10 \\
<10\end{array}$ \\
\hline 4 & $\begin{array}{l}02-28-86 \\
05-15-86 \\
07-30-86 \\
09-03-86\end{array}$ & $\begin{array}{l}0950 \\
1610 \\
1300 \\
1430\end{array}$ & $\begin{array}{r}<1 \\
2 \\
2 \\
2\end{array}$ & $\begin{array}{r}290 \\
99 \\
62 \\
62\end{array}$ & $\begin{array}{l}- \\
150 \\
170 \\
170\end{array}$ & $\begin{array}{r}<1 \\
<1 \\
<1 \\
1\end{array}$ & $\begin{array}{l}<10 \\
<10 \\
<10 \\
<10\end{array}$ & $\begin{array}{l}<10 \\
<10 \\
<10 \\
<10\end{array}$ \\
\hline 5 & $\begin{array}{l}11-19-85 \\
01-29-86 \\
03-19-86 \\
05-15-86 \\
07-30-86 \\
09-03-86\end{array}$ & $\begin{array}{l}1145 \\
1115 \\
1400 \\
1040 \\
1000 \\
1245\end{array}$ & $\begin{array}{l}14 \\
12 \\
15 \\
16 \\
14 \\
14\end{array}$ & $\begin{array}{r}100 \\
100 \\
100 \\
95 \\
99 \\
93\end{array}$ & $\begin{array}{l}40 \\
40 \\
50 \\
40 \\
40\end{array}$ & $\begin{array}{l}<1 \\
<1 \\
<1 \\
<1 \\
<1 \\
1\end{array}$ & $\begin{array}{l}<10 \\
<10 \\
<10 \\
<10 \\
<10 \\
<10\end{array}$ & $\begin{array}{l}<10 \\
<10 \\
<10 \\
<10 \\
<10 \\
<10\end{array}$ \\
\hline 6 & $\begin{array}{l}11-15-85 \\
01-27-86 \\
03-19-86 \\
05-12-86 \\
08-04-86 \\
09-03-86\end{array}$ & $\begin{array}{l}1035 \\
0930 \\
1100 \\
1040 \\
1010 \\
1015\end{array}$ & $\begin{array}{l}<1 \\
<1 \\
<1 \\
<1 \\
<1 \\
<1\end{array}$ & $\begin{array}{l}83 \\
83 \\
83 \\
77 \\
71 \\
74\end{array}$ & $\begin{array}{r}<20 \\
<20 \\
20 \\
20 \\
20\end{array}$ & $\begin{array}{r}<1 \\
<1 \\
<1 \\
<1 \\
<1 \\
1\end{array}$ & $\begin{array}{l}<10 \\
<10 \\
<10 \\
<10 \\
<10 \\
<10\end{array}$ & $\begin{array}{r}<10 \\
<10 \\
10 \\
<10 \\
<10 \\
10\end{array}$ \\
\hline
\end{tabular}


TABLE 7.--Data on trace elements--Continued

\begin{tabular}{|c|c|c|c|c|c|c|c|c|}
\hline $\begin{array}{l}\text { SITE NUMBER } \\
\text { (figure 2) }\end{array}$ & DATE & $\begin{array}{l}\text { IRON, } \\
\text { DIS- } \\
\text { SOLVED } \\
\text { (UG/L } \\
\text { AS FE) }\end{array}$ & $\begin{array}{l}\text { LEAD, } \\
\text { DIS- } \\
\text { SOLVED } \\
\text { (UG/L } \\
\text { AS PB) }\end{array}$ & $\begin{array}{l}\text { MANGA- } \\
\text { NESE, } \\
\text { DIS- } \\
\text { SOLVED } \\
\text { (UG/L } \\
\text { AS MN) }\end{array}$ & $\begin{array}{l}\text { MERCURY, } \\
\text { DIS- } \\
\text { SOLVED } \\
\text { (UG/L } \\
\text { AS HG) }\end{array}$ & $\begin{array}{l}\text { SELE- } \\
\text { NIUM, } \\
\text { DIS- } \\
\text { SOLVED } \\
\text { (UG/L } \\
\text { AS SE) }\end{array}$ & $\begin{array}{l}\text { SILVER, } \\
\text { DIS- } \\
\text { SOLVED } \\
\text { (UG/L } \\
\text { AS AG) }\end{array}$ & $\begin{array}{l}\text { ZINC, } \\
\text { DIS- } \\
\text { SOLVED } \\
\text { (UG/L } \\
\text { AS } \mathrm{ZN} \text { ) }\end{array}$ \\
\hline 1 & $\begin{array}{l}12-19-85 \\
01-31-86 \\
03-17-86 \\
05-09-86 \\
08-06-86 \\
09-04-86\end{array}$ & $\begin{array}{r}4 \\
6 \\
8 \\
<3 \\
9 \\
6\end{array}$ & $\begin{array}{l}<10 \\
<10 \\
<10 \\
<10 \\
<10 \\
<10\end{array}$ & $\begin{array}{r}<1 \\
<1 \\
<1 \\
<1 \\
2 \\
1\end{array}$ & $\begin{array}{l}<0.1 \\
<.1 \\
<.1 \\
<.1 \\
<.1 \\
<.1\end{array}$ & $\begin{array}{r}<1 \\
<1 \\
1 \\
<1 \\
<1 \\
<1\end{array}$ & $\begin{array}{l}<1 \\
<1 \\
<1 \\
<1 \\
<1 \\
<1\end{array}$ & $\begin{array}{r}16 \\
14 \\
10 \\
4 \\
9 \\
5\end{array}$ \\
\hline 2 & $\begin{array}{l}11-18-85 \\
01-27-86 \\
03-17-86 \\
05-14-86\end{array}$ & $\begin{array}{l}<3 \\
<3 \\
<3 \\
<3\end{array}$ & $\begin{array}{l}<10 \\
<10 \\
<10 \\
<10\end{array}$ & $\begin{array}{l}<1 \\
<1 \\
<1 \\
<1\end{array}$ & $\begin{array}{l}<.1 \\
<.1 \\
<.1 \\
<.1\end{array}$ & $\begin{array}{r}<1 \\
<1 \\
1 \\
<1\end{array}$ & $\begin{array}{l}<1 \\
<1 \\
<1 \\
<1\end{array}$ & $\begin{array}{l}<3 \\
<3 \\
<3 \\
<3\end{array}$ \\
\hline 3 & $\begin{array}{l}11-18-85 \\
01-28-86 \\
03-17-86 \\
05-16-86 \\
07-31-86 \\
09-04-86\end{array}$ & $\begin{array}{l}<3 \\
<3 \\
<3 \\
<3 \\
<3 \\
<3\end{array}$ & $\begin{array}{r}<10 \\
<10 \\
10 \\
<10 \\
<10 \\
<10\end{array}$ & $\begin{array}{r}<1 \\
<1 \\
<1 \\
<1 \\
<1 \\
1\end{array}$ & $\begin{array}{l}<.1 \\
<.1 \\
<.1 \\
<.1 \\
<.1 \\
<.1\end{array}$ & $\begin{array}{r}<1 \\
<1 \\
2 \\
<1 \\
<1 \\
<1\end{array}$ & $\begin{array}{l}<1 \\
<1 \\
<1 \\
<1 \\
<1 \\
<1\end{array}$ & $\begin{array}{r}11 \\
10 \\
4 \\
5 \\
<3 \\
5\end{array}$ \\
\hline 4 & $\begin{array}{l}02-28-86 \\
05-15-86 \\
07-30-86 \\
09-03-86\end{array}$ & $\begin{array}{r}230 \\
630 \\
32 \\
19\end{array}$ & $\begin{array}{l}<10 \\
<10 \\
<10 \\
<10\end{array}$ & $\begin{array}{r}520 \\
250 \\
13 \\
7\end{array}$ & $\begin{array}{l}.1 \\
<.1 \\
<.1 \\
<.1\end{array}$ & $\begin{array}{l}<1 \\
<1 \\
<1 \\
<1\end{array}$ & $\begin{array}{l}<1 \\
<1 \\
<1 \\
<1\end{array}$ & $\begin{array}{r}20 \\
5 \\
4 \\
20\end{array}$ \\
\hline 5 & $\begin{array}{l}11-19-85 \\
01-29-86 \\
03-19-86 \\
05-15-86 \\
07-30-86 \\
09-03-86\end{array}$ & $\begin{array}{r}8 \\
13 \\
5 \\
<3 \\
14 \\
6\end{array}$ & $\begin{array}{l}<10 \\
<10 \\
<10 \\
<10 \\
<10 \\
<10\end{array}$ & $\begin{array}{r}<1 \\
8 \\
<1 \\
<1 \\
3 \\
2\end{array}$ & $\begin{array}{l}<.1 \\
<.1 \\
<.1 \\
<.1 \\
<.1 \\
<.1\end{array}$ & $\begin{array}{l}2 \\
2 \\
2 \\
2 \\
2 \\
2\end{array}$ & $\begin{array}{l}<1 \\
<1 \\
<1 \\
<1 \\
<1 \\
<1\end{array}$ & $\begin{array}{r}64 \\
170 \\
41 \\
14 \\
66 \\
32\end{array}$ \\
\hline 6 & $\begin{array}{l}11-15-85 \\
01-27-86 \\
03-19-86 \\
05-12-86 \\
08-04-86 \\
09-03-86\end{array}$ & $\begin{array}{r}9 \\
8 \\
9 \\
41 \\
7 \\
6\end{array}$ & $\begin{array}{l}<10 \\
<10 \\
<10 \\
<10 \\
<10 \\
<10\end{array}$ & $\begin{array}{r}<1 \\
1 \\
<1 \\
<1 \\
<1 \\
1\end{array}$ & $\begin{array}{l}<.1 \\
<.1 \\
<.1 \\
<.1 \\
<.1 \\
<.1\end{array}$ & $\begin{array}{r}<1 \\
<1 \\
2 \\
<1 \\
<1 \\
<1\end{array}$ & $\begin{array}{r}<1 \\
<1 \\
1 \\
<1 \\
<1 \\
<1\end{array}$ & $\begin{array}{r}9 \\
14 \\
11 \\
24 \\
<3 \\
5\end{array}$ \\
\hline
\end{tabular}


TABLE 7.--Data on trace elements--Continued

\begin{tabular}{|c|c|c|c|c|c|c|c|c|}
\hline $\begin{array}{l}\text { SITE NUMBER } \\
\text { (f1gure 2) }\end{array}$ & DATE & TIME & $\begin{array}{l}\text { ARSENIC, } \\
\text { DIS- } \\
\text { SOLVED } \\
\text { (UG/L } \\
\text { AS AS) }\end{array}$ & $\begin{array}{c}\text { BARIUM, } \\
\text { DIS- } \\
\text { SOLVED } \\
\text { (UG/L } \\
\text { AS BA) }\end{array}$ & $\begin{array}{c}\text { BORON, } \\
\text { DIS- } \\
\text { SOLVED } \\
\text { (UG/L } \\
\text { AS B) }\end{array}$ & $\begin{array}{l}\text { CADMIUM, } \\
\text { DIS- } \\
\text { SOLVED } \\
\text { (UG/L } \\
\text { AS CD) }\end{array}$ & $\begin{array}{l}\text { CHRO- } \\
\text { MIUM, } \\
\text { DIS- } \\
\text { SOLVED } \\
\text { (UG/L } \\
\text { AS CR) }\end{array}$ & $\begin{array}{l}\text { COPPER, } \\
\text { DIS- } \\
\text { SOLVED } \\
\text { (UG/L } \\
\text { AS CU) }\end{array}$ \\
\hline $\begin{array}{r}7 \\
8 \\
9 \\
10 \\
11\end{array}$ & $\begin{array}{l}05-09-86 \\
05-09-86 \\
05-09-86 \\
05-19-86 \\
05-20-86\end{array}$ & $\begin{array}{l}1240 \\
1450 \\
1600 \\
1150 \\
1430\end{array}$ & $\begin{array}{r}4 \\
3 \\
<1 \\
22 \\
18\end{array}$ & $\begin{array}{r}130 \\
26 \\
14 \\
<100 \\
48\end{array}$ & $\begin{array}{r}170 \\
220 \\
10 \\
2,400 \\
210\end{array}$ & $\begin{array}{l}<1 \\
<1 \\
<1 \\
10 \\
<1\end{array}$ & $\begin{array}{l}<10 \\
<10 \\
<10 \\
<10 \\
<10\end{array}$ & $\begin{array}{r}<10 \\
<10 \\
<10 \\
10 \\
<10\end{array}$ \\
\hline $\begin{array}{l}12 \\
13 \\
14 \\
15 \\
16\end{array}$ & $\begin{array}{l}05-20-86 \\
05-20-86 \\
05-13-86 \\
05-13-86 \\
05-13-86\end{array}$ & $\begin{array}{l}1230 \\
1000 \\
1200 \\
1610 \\
1350\end{array}$ & $\begin{array}{l}23 \\
25 \\
<1 \\
29 \\
<1\end{array}$ & $\begin{array}{l}40 \\
10 \\
28 \\
48 \\
21\end{array}$ & $\begin{array}{r}150 \\
160 \\
20 \\
160 \\
10\end{array}$ & $\begin{array}{l}<1 \\
<1 \\
<1 \\
<1 \\
<1\end{array}$ & $\begin{array}{l}<10 \\
<10 \\
<10 \\
<10 \\
<10\end{array}$ & $\begin{array}{r}10 \\
<10 \\
<10 \\
<10 \\
<10\end{array}$ \\
\hline $\begin{array}{l}17 \\
18 \\
19 \\
20 \\
21\end{array}$ & $\begin{array}{l}05-13-86 \\
05-19-86 \\
05-14-86 \\
05-16-86 \\
05-15-86\end{array}$ & $\begin{array}{l}1010 \\
1510 \\
1240 \\
1240 \\
1410\end{array}$ & $\begin{array}{r}12 \\
8 \\
2 \\
9 \\
7\end{array}$ & $\begin{array}{r}58 \\
81 \\
170 \\
64 \\
73\end{array}$ & $\begin{array}{r}80 \\
60 \\
160 \\
110 \\
90\end{array}$ & $\begin{array}{l}<1 \\
<1 \\
<1 \\
<1 \\
<1\end{array}$ & $\begin{array}{l}<10 \\
<10 \\
<10 \\
<10 \\
<10\end{array}$ & $\begin{array}{l}<10 \\
<10 \\
<10 \\
<10 \\
<10\end{array}$ \\
\hline $\begin{array}{l}22 \\
23 \\
24 \\
25 \\
26\end{array}$ & $\begin{array}{l}05-14-86 \\
05-15-86 \\
05-14-86 \\
05-12-86 \\
05-12-86\end{array}$ & $\begin{array}{l}1630 \\
1200 \\
1745 \\
1235 \\
1430\end{array}$ & $\begin{array}{r}3 \\
4 \\
3 \\
21 \\
15\end{array}$ & $\begin{array}{r}85 \\
130 \\
12 \\
73 \\
120\end{array}$ & $\begin{array}{l}80 \\
60 \\
50 \\
20 \\
20\end{array}$ & $\begin{array}{l}<1 \\
<1 \\
<1 \\
<1 \\
<1\end{array}$ & $\begin{array}{l}<10 \\
<10 \\
<10 \\
<10 \\
<10\end{array}$ & $\begin{array}{l}<10 \\
<10 \\
<10 \\
<10 \\
<10\end{array}$ \\
\hline $\begin{array}{l}27 \\
28 \\
30 \\
31 \\
32 \\
33\end{array}$ & $\begin{array}{l}09-02-86 \\
08-26-86 \\
08-19-86 \\
09-04-86 \\
09-05-86 \\
11-26-85\end{array}$ & $\begin{array}{l}1545 \\
1400 \\
1430 \\
1340 \\
1445 \\
1055\end{array}$ & $\begin{array}{r}43 \\
55 \\
38 \\
21 \\
19 \\
6\end{array}$ & $\begin{array}{r}140 \\
56 \\
24 \\
97 \\
100 \\
63\end{array}$ & $\begin{array}{l}190 \\
260 \\
170 \\
290 \\
410 \\
180\end{array}$ & $\begin{array}{r}1 \\
<1 \\
<1 \\
1 \\
1 \\
<1\end{array}$ & $\begin{array}{l}<10 \\
<10 \\
<10 \\
<10 \\
<10 \\
<10\end{array}$ & $\begin{array}{r}<10 \\
<10 \\
<10 \\
<10 \\
<10 \\
10\end{array}$ \\
\hline
\end{tabular}


TABLE 7.--Data on trace elements--Cont Inued

\begin{tabular}{|c|c|c|c|c|c|c|c|c|}
\hline $\begin{array}{l}\text { SITE NUMBER } \\
\text { (f1gure 2) }\end{array}$ & DATE & $\begin{array}{r}\text { IRON, } \\
\text { DIS- } \\
\text { SOLVED } \\
\text { (UG/L } \\
\text { AS FE) }\end{array}$ & $\begin{array}{l}\text { LEAD, } \\
\text { DIS- } \\
\text { SOLVED } \\
\text { (UG/L } \\
\text { AS PB) }\end{array}$ & $\begin{array}{l}\text { MANGA- } \\
\text { NESE, } \\
\text { DIS- } \\
\text { SOLVED } \\
\text { (UG/L } \\
\text { AS MN) }\end{array}$ & $\begin{array}{l}\text { MERCURY, } \\
\text { DIS- } \\
\text { SOLVED } \\
\text { (UG/L } \\
\text { AS HG) }\end{array}$ & $\begin{array}{l}\text { SELE- } \\
\text { NIUM, } \\
\text { DIS- } \\
\text { SOLVED } \\
\text { (UG/L } \\
\text { AS SE) }\end{array}$ & $\begin{array}{l}\text { SILVER, } \\
\text { DIS- } \\
\text { SOLVED } \\
\text { (UG/L } \\
\text { AS AG) }\end{array}$ & $\begin{array}{l}\text { ZINC, } \\
\text { DIS- } \\
\text { SOLVED } \\
\text { (UG/L } \\
\text { AS } 2 N \text { ) }\end{array}$ \\
\hline $\begin{array}{r}7 \\
8 \\
9 \\
10 \\
11\end{array}$ & $\begin{array}{l}05-09-86 \\
05-09-86 \\
05-09-86 \\
05-19-86 \\
05-20-86\end{array}$ & $\begin{array}{r}<3 \\
11 \\
<3 \\
11,000 \\
4\end{array}$ & $\begin{array}{r}10 \\
<10 \\
<10 \\
<100 \\
<10\end{array}$ & $\begin{array}{r}<1 \\
<1 \\
<1 \\
1,000 \\
<1\end{array}$ & $\begin{array}{l}<0.1 \\
<.1 \\
<.1 \\
<.1 \\
<.1\end{array}$ & $\begin{array}{l}<1 \\
<1 \\
<1 \\
<1 \\
<1\end{array}$ & $\begin{array}{l}<1 \\
<1 \\
<1 \\
<1 \\
<1\end{array}$ & $\begin{array}{r}53 \\
21 \\
4 \\
10 \\
28\end{array}$ \\
\hline $\begin{array}{l}12 \\
13 \\
14 \\
15 \\
16\end{array}$ & $\begin{array}{l}05-20-86 \\
05-20-86 \\
05-13-86 \\
05-13-86 \\
05-13-86\end{array}$ & $\begin{array}{r}<3 \\
6 \\
19 \\
16 \\
18\end{array}$ & $\begin{array}{l}<10 \\
<10 \\
<10 \\
<10 \\
<10\end{array}$ & $\begin{array}{l}<1 \\
<1 \\
<1 \\
36 \\
<1\end{array}$ & $\begin{array}{l}<.1 \\
<.1 \\
<.1 \\
<.1 \\
<.1\end{array}$ & $\begin{array}{l}<1 \\
<1 \\
1 \\
<1 \\
<1\end{array}$ & $\begin{array}{l}<1 \\
<1 \\
<1 \\
<1 \\
<1\end{array}$ & $\begin{array}{r}32 \\
23 \\
88 \\
8 \\
23\end{array}$ \\
\hline $\begin{array}{l}17 \\
18 \\
19 \\
20 \\
21\end{array}$ & $\begin{array}{l}05-13-86 \\
05-19-86 \\
05-14-86 \\
05-16-86 \\
05-15-86\end{array}$ & $\begin{array}{r}48 \\
5 \\
<3 \\
<3 \\
<3\end{array}$ & $\begin{array}{l}<10 \\
<10 \\
<10 \\
<10 \\
<10\end{array}$ & $\begin{array}{l}66 \\
<1 \\
<1 \\
<1 \\
<1\end{array}$ & $\begin{array}{l}<.1 \\
<.1 \\
<.1 \\
<.1 \\
<.1\end{array}$ & $\begin{array}{l}<1 \\
<1 \\
<1 \\
<1 \\
<1\end{array}$ & $\begin{array}{l}<1 \\
<1 \\
<1 \\
<1 \\
<1\end{array}$ & $\begin{array}{r}4 \\
18 \\
66 \\
18 \\
25\end{array}$ \\
\hline $\begin{array}{l}22 \\
23 \\
24 \\
25 \\
26\end{array}$ & $\begin{array}{l}05-14-86 \\
05-15-86 \\
05-14-86 \\
05-12-86 \\
05-12-86\end{array}$ & $\begin{array}{r}5 \\
<3 \\
65 \\
9 \\
10\end{array}$ & $\begin{array}{l}<10 \\
<10 \\
<10 \\
<10 \\
<10\end{array}$ & $\begin{array}{l}<1 \\
<1 \\
15 \\
<1 \\
3\end{array}$ & $\begin{array}{l}<.1 \\
<.1 \\
<.1 \\
<.1 \\
<.1\end{array}$ & $\begin{array}{r}<1 \\
4 \\
<1 \\
<1 \\
<1\end{array}$ & $\begin{array}{l}<1 \\
<1 \\
<1 \\
<1 \\
<1\end{array}$ & $\begin{array}{r}99 \\
13 \\
28 \\
120 \\
380\end{array}$ \\
\hline $\begin{array}{l}27 \\
28 \\
30 \\
31 \\
32 \\
33\end{array}$ & $\begin{array}{l}09-02-86 \\
08-26-86 \\
08-19-86 \\
09-04-86 \\
09-05-86 \\
11-26-85\end{array}$ & $\begin{array}{r}870 \\
210 \\
6 \\
190 \\
250 \\
12\end{array}$ & $\begin{array}{l}<10 \\
<10 \\
<10 \\
<10 \\
<10 \\
<10\end{array}$ & $\begin{array}{r}490 \\
570 \\
3 \\
270 \\
520 \\
4\end{array}$ & $\begin{array}{l}<.1 \\
<.1 \\
<.1 \\
<.1 \\
<.1 \\
<.1\end{array}$ & $\begin{array}{l}<1 \\
<1 \\
<1 \\
<1 \\
<1 \\
<1\end{array}$ & $\begin{array}{l}<1 \\
<1 \\
<1 \\
<1 \\
<1 \\
<1\end{array}$ & $\begin{array}{r}3 \\
<3 \\
10 \\
<3 \\
4 \\
<3\end{array}$ \\
\hline
\end{tabular}


TABLE 8.--Data on organic compounds 1

\begin{tabular}{|c|c|c|c|}
\hline $\begin{array}{l}\text { Site number } \\
(\text { figure } 2)^{2}\end{array}$ & Date & $\begin{array}{c}\text { Estimated } \\
\text { combined concentration } \\
\text { of organic compounds } \\
\text { (micrograms per liter) }\end{array}$ & $\begin{array}{c}\text { Number of } \\
\text { organic compounds } \\
\text { detected }\end{array}$ \\
\hline 1 & $08-06-86$ & 0 & 0 \\
\hline 3 & $07-31-86$ & 2 & 1 \\
\hline 4 & $07-30-86$ & 6 & 1 \\
\hline 5 & $07-30-86$ & 17 & 1 \\
\hline 6 & $08-04-86$ & 4 & 1 \\
\hline 7 & $08-11-86$ & 0 & 0 \\
\hline 8 & $08-19-86$ & 0 & 0 \\
\hline 9 & $08-12-86$ & 0 & 0 \\
\hline $10 *$ & $08-13-86$ & 17 & 2 \\
\hline 11 & $08-13-86$ & 0 & 0 \\
\hline 12 & $08-13-86$ & 0 & 0 \\
\hline 13 & $08-27-86$ & 4 & 1 \\
\hline 14 & $08-18-86$ & 0 & 0 \\
\hline 16 & $08-18-86$ & 0 & 0 \\
\hline 17 & $08-18-86$ & 3 & 1 \\
\hline 18 & $08-26-86$ & 3 & 1 \\
\hline 19 & $08-12-86$ & 0 & 0 \\
\hline 20 & $08-21-86$ & 3 & 1 \\
\hline 21 & $08-21-86$ & 0 & 0 \\
\hline 22 & $08-21-86$ & 0 & 0 \\
\hline 23 & $08-21-86$ & 0 & 0 \\
\hline 24 & $08-12-86$ & 0 & 0 \\
\hline 25 & $08-01-86$ & 19 & 1 \\
\hline 26 & $08-01-86$ & 0 & 0 \\
\hline $27 *$ & $09-02-86$ & 40 & 6 \\
\hline $28 *$ & $08-26-86$ & 19 & 5 \\
\hline 29 & $08-19-86$ & 180 & 47 \\
\hline 30 & $08-19-86$ & 0 & 0 \\
\hline $31 *$ & $09-04-86$ & 40 & 5 \\
\hline $32 *$ & $09-05-86$ & 0 & 0 \\
\hline
\end{tabular}

1 Semiquantitative analysis by the U.S. Geological Survey National Water Quality Laboratory, Denver, Colo., determined by gas chromatograph using a flame-ionization detector. See text for details.

2 Asterisk indicates that well-casing material is polyvinyl chloride (PVC), which can contribute organic compounds to water s amples. 
TABLE 9.--Data on dissolved radium-226, radon-222, and uranium ["-, " no data available]

\begin{tabular}{|c|c|c|c|c|}
\hline $\begin{array}{l}\text { Site number } \\
\text { (figure 2) }\end{array}$ & Date & $\begin{array}{l}\text { Radium-226 } \\
\text { (picocuries } \\
\text { per liter) }\end{array}$ & $\begin{array}{l}\text { Radon- } 222 \\
\text { (picocuries } \\
\text { per liter) }\end{array}$ & $\begin{array}{l}\text { Uranium } \\
\text { (micrograms } \\
\text { per liter) }\end{array}$ \\
\hline 1 & $08-06-86$ & 0.05 & 610 & 3.0 \\
\hline 5 & $07-30-86$ & .06 & 600 & 1.2 \\
\hline 6 & $08-04-86$ & .06 & 340 & .68 \\
\hline 9 & $08-12-86$ & .04 & 900 & -. \\
\hline 13 & $08-27-86$ & .04 & 580 & .94 \\
\hline 14 & $08-18-86$ & .09 & 700 & 1.3 \\
\hline 17 & $08-18-86$ & .07 & 550 & 2.7 \\
\hline 18 & $08-26-86$ & $\ldots$ & 670 & - \\
\hline 24 & $08-12-86$ & .56 & 5,000 & 5.3 \\
\hline 26 & $08-01-86$ & .10 & 840 & 1.2 \\
\hline
\end{tabular}


TABLE 10.-Statistical summary of water-quality data ${ }^{1}$

[Abbreviations: mg/L, milligrams per liter; n.a., not applicable; NTu, nephelometric turbidity units; $\mathrm{pCi} / \mathrm{L}$, picocuries per liter; res. evap., residue remaining after evaporation at 180 'C; DS, dissolved solids; $\mathrm{kg} / \mathrm{L}$, micrograms per liter; $\mu \mathrm{S} / \mathrm{cm}$, microsiemens per centimeter at $25{ }^{\circ} \mathrm{C}$; ${ }^{\circ} \mathrm{C}$, degrees Celsius]

\begin{tabular}{|c|c|c|c|c|c|c|c|c|c|}
\hline \multirow[b]{3}{*}{ Constituent or property } & \multirow[b]{3}{*}{$\begin{array}{l}\text { Mean } \\
\text { value }\end{array}$} & \multirow[b]{3}{*}{$\begin{array}{l}\text { Standard } \\
\text { deviation }\end{array}$} & \multirow[b]{3}{*}{$\begin{array}{l}\text { Median } \\
\text { value }\end{array}$} & \multirow{2}{*}{\multicolumn{2}{|c|}{ Minimum }} & \multirow{2}{*}{\multicolumn{2}{|c|}{ Msxi mum }} & \multirow{2}{*}{\multicolumn{2}{|c|}{$\begin{array}{l}\text { Number of } \\
\text { measurement } 8\end{array}$}} \\
\hline & & & & & & & & & \\
\hline & & & & Value & $\begin{array}{l}\text { Site } \\
\text { number }{ }^{2}\end{array}$ & Value & $\begin{array}{c}\text { Site } \\
\text { number }\end{array}$ & $\begin{array}{l}\text { Used in } \\
\text { calcula- } \\
\text { tions }\end{array}$ & $\begin{array}{l}\text { Below } \\
\text { detection } \\
\text { limit }\end{array}$ \\
\hline $\begin{array}{l}\text { Specific conductance }(\mu \mathrm{S} / \mathrm{cm}) \\
\text { pH (standard units) } \\
\text { Water temperature ( } \\
\text { Turbidity (NTU) } \\
\text { Hardness (mg/L) }\end{array}$ & $\begin{array}{l}450 \\
\text { n.a. } \\
15 \\
4.5 \\
130\end{array}$ & $\begin{array}{r}485 \\
\mathrm{n.a} \\
2.6 \\
18.1 \\
104\end{array}$ & $100^{355} 7.7$ & $11^{105} 6.6$ & $\begin{array}{l}24 \\
10 \\
19 \\
17 \\
24\end{array}$ & $\begin{array}{c}3,000 \\
9.1 \\
22 \\
120 \\
570\end{array}$ & $\begin{array}{r}10 \\
1 \\
5 \\
10 \\
10\end{array}$ & $\begin{array}{r}119 \\
119 \\
117 \\
99 \\
116\end{array}$ & $\begin{array}{l}0 \\
0 \\
0 \\
0 \\
0\end{array}$ \\
\hline $\begin{array}{l}\text { Calcium (ng/L) } \\
\text { Magnesium (mg/L) } \\
\text { Sodium (mg/L) } \\
\text { Sodium (percenc) } \\
\text { Sodium adsorption ratio }\end{array}$ & $\begin{array}{r}40.4 \\
7.5 \\
44.3 \\
38 \\
1.8\end{array}$ & $\begin{array}{l}37 \\
5.4 \\
84.3 \\
21 \\
1.9\end{array}$ & $\begin{array}{r}31.0 \\
5.7 \\
23.0 \\
31 \\
1.0\end{array}$ & $\begin{array}{r}5.0 \\
.1 \\
9.4 \\
12 \\
.4\end{array}$ & $\begin{array}{r}24 \\
9 \\
22 \\
26 \\
26\end{array}$ & $\begin{array}{r}220 \\
23 \\
510 \\
84 \\
10\end{array}$ & $\begin{array}{r}10 \\
31 \\
10 \\
1 \\
10\end{array}$ & $\begin{array}{l}117 \\
116 \\
117 \\
116 \\
116\end{array}$ & $\begin{array}{l}0 \\
1 \\
0 \\
0 \\
0\end{array}$ \\
\hline $\begin{array}{l}\text { Potassium (mg/L) } \\
\text { Bicarbonate (mg/L) } \\
\text { Carbonate (mg/L) } \\
\text { Alkalinity (CaCO3; mg/L) } \\
\text { Sulfate (mg/L) }\end{array}$ & $\begin{array}{c}2.7 \\
156 \\
0.5 \\
129 \\
78\end{array}$ & $\begin{array}{c}1 \\
66 \\
53 \\
242\end{array}$ & $\begin{array}{c}2.5 \\
150 \\
0 \\
122 \\
23\end{array}$ & $\begin{array}{r}333^{.6} \\
27 \\
.7\end{array}$ & $\begin{array}{r}1 \\
24 \\
\star \\
24 \\
9\end{array}$ & $\begin{array}{l}6.0 \\
412 \\
10 \\
338 \\
1,400\end{array}$ & $26, \begin{array}{r}10 \\
32 \\
1 \\
32 \\
10\end{array}$ & $\begin{array}{l}117 \\
118 \\
118 \\
118 \\
117\end{array}$ & $\begin{array}{l}0 \\
0 \\
0 \\
0 \\
0\end{array}$ \\
\hline $\begin{array}{l}\text { Chloride (mg/L) } \\
\text { Fluoride (mg/L) } \\
\text { S111ca; mg/L) } \\
\text { DS (res. evap.; mg/L) } \\
\text { NO2-N (mg/L) }\end{array}$ & $\begin{array}{r}14.9 \\
. .5 \\
299 \\
.02\end{array}$ & $\begin{array}{l}23.5 \\
15 \\
362 \\
.02\end{array}$ & $\begin{array}{r}8.8 \\
33^{.2} \\
.01\end{array}$ & $\begin{array}{l}1.0 \\
17^{.1} \\
78 \\
.01\end{array}$ & $\begin{array}{l}9 \\
\star \\
9 \\
9 \\
\star\end{array}$ & $\begin{array}{c}150 \\
5.4 \\
80 \\
2,430 \\
.06\end{array}$ & $\begin{array}{l}10 \\
10 \\
13 \\
10 \\
32\end{array}$ & $\begin{array}{r}117 \\
83 \\
117 \\
117 \\
16\end{array}$ & $\begin{array}{r}0 \\
34 \\
0 \\
0 \\
101\end{array}$ \\
\hline $\begin{array}{l}\text { NO2+NO3 (mg/L) } \\
\text { NH4-N (mg/L) } \\
\text { NH4torganlc-N (mg/L) } \\
\text { N1trogen-N (mg/L) } \\
\text { Phosphorus-P (mg/L) }\end{array}$ & $\begin{array}{l}2.0 \\
.06 \\
.4 \\
1.9 \\
.09\end{array}$ & $\begin{array}{l}3.4 \\
.15 \\
.2 \\
1.6 \\
.08\end{array}$ & $\begin{array}{l}1.0 \\
.02 \\
.3 \\
1.4 \\
.06\end{array}$ & $\begin{array}{l}.1 \\
.01 \\
.2 \\
.3 \\
.02\end{array}$ & $\begin{array}{r}17 \\
\star \\
\star \\
17 \\
\star\end{array}$ & $\begin{array}{c}21 \\
1.2 \\
1.6 \\
6.6 \\
.27\end{array}$ & $\begin{array}{l}7 \\
31 \\
31 \\
26 \\
17\end{array}$ & $\begin{array}{r}102 \\
81 \\
99 \\
34 \\
25\end{array}$ & $\begin{array}{r}15 \\
36 \\
17 \\
0 \\
1\end{array}$ \\
\hline $\begin{array}{l}\text { Orthophosphate-P (mg/L) } \\
\text { Arsenic ( } \mu \mathrm{g} / \mathrm{L}) \\
\text { Barium ( } \mu \mathrm{g} / \mathrm{L}) \\
\text { Boron ( } \mathrm{g}_{\mathrm{g} / \mathrm{L})} \\
\text { Cadmium }(\mu \mathrm{g} / \mathrm{L})\end{array}$ & $\begin{array}{r}12^{.05} \\
80 \\
170 \\
2\end{array}$ & $\begin{array}{r}11 \\
49 \\
330 \\
3\end{array}$ & $\begin{array}{r}10^{.05} \\
73 \\
130 \\
1\end{array}$ & $\begin{array}{l}1 \\
10^{.01} \\
1\end{array}$ & $\begin{array}{r}24,9 \\
3 \\
13 \\
9,6 \\
\star\end{array}$ & $\begin{array}{c}55^{.14} \\
290 \\
2,400 \\
10\end{array}$ & $\begin{array}{r}20 \\
28 \\
4 \\
10 \\
10\end{array}$ & $\begin{array}{r}25 \\
48 \\
57 \\
51 \\
8\end{array}$ & $\begin{array}{r}1 \\
10 \\
1 \\
1 \\
50\end{array}$ \\
\hline $\begin{array}{l}\text { Chromium }(\mu g / L) \\
\text { Copper }(\mu g / L) \\
\text { Iron }(\mu g / L) \\
\text { Lead }(\mu g / L) \\
\text { Manganese }(\mu g / L)\end{array}$ & $\begin{array}{r}15 \\
10 \\
354 \\
10 \\
165\end{array}$ & $\begin{array}{r}7 \\
0 \\
1,758 \\
0 \\
272\end{array}$ & $\begin{array}{r}15 \\
10 \\
10 \\
10 \\
8\end{array}$ & $\begin{array}{r}10 \\
10 \\
4 \\
10 \\
1\end{array}$ & $\begin{array}{l}3 \\
\star \\
1 \\
\star \\
\star\end{array}$ & $\begin{array}{r}20 \\
10 \\
11,000 \\
10 \\
1,000\end{array}$ & $\begin{array}{r}1 \\
\star \\
10 \\
\star \\
10\end{array}$ & $\begin{array}{r}2 \\
6 \\
39 \\
2 \\
23\end{array}$ & $\begin{array}{l}56 \\
52 \\
19 \\
56 \\
35\end{array}$ \\
\hline $\begin{array}{l}\text { Mercury }(\mu g / L) \\
\text { Selenium }(\mu g / L) \\
\text { S1lver }(\mu g / L) \\
\text { Zinc }(\mu g / L) \\
\text { Radium-226 (pCI/L) }\end{array}$ & $2_{34}^{.1}$ & $0^{0} .8$ & $2^{.1} 4^{.06}$ & $\begin{array}{ll}1^{.1} & \\
1 & \\
.04\end{array}$ & $\begin{array}{r}\star \\
\star \\
\star \\
27 \\
9\end{array}$ & ${ }_{380}^{4^{1}}$ & $\begin{array}{r}\star \\
23 \\
\star \\
20 \\
24\end{array}$ & $\begin{array}{r}1 \\
12 \\
1 \\
49 \\
9\end{array}$ & $\begin{array}{r}57 \\
46 \\
57 \\
9 \\
0\end{array}$ \\
\hline $\begin{array}{l}\text { Redon }(\mathrm{pCl} / \mathrm{L}) \\
\text { Uranlum }(\mu \mathrm{g} / \mathrm{L}) \\
\text { Dissolved organlc carbon }(\mathrm{mg} / \mathrm{L})\end{array}$ & $\begin{aligned} & 1,080 \\
& 2 . 04 \\
& .9\end{aligned}$ & $\begin{array}{r}1.393 \\
1.56 \\
.8\end{array}$ & $\begin{array}{c}642 \\
1.25 \\
.6\end{array}$ & $\begin{array}{c}339 \\
.68 \\
.2\end{array}$ & $\begin{array}{l}6 \\
6 \\
1\end{array}$ & $\begin{array}{r}5,020 \\
5.3 \\
6.3\end{array}$ & $\begin{array}{l}24 \\
24 \\
31\end{array}$ & $\begin{array}{r}10 \\
8 \\
117\end{array}$ & $\begin{array}{l}0 \\
0 \\
0\end{array}$ \\
\hline
\end{tabular}

1 Measurements below detection limit were not included when summary statistics were calculated; thus, these statistical estimates may be skewed higher than the true population statistics for constituents and properties having values below detection limit.

2 Asterisk indicates multiple occurrences of this value. 


\section{REFERENCES CITED}

Barcelona, M.J., Gibb, J.P., and Miller, R.A., 1983, A guide to the selection of materials for monitoring well construction and ground-water sampling: Champaign, Ill., Illinois State Water Survey, ISWS Contract Report 327,78 p.

Barnes, Ivan, 1964, Field measurement of alkalinity and $\mathrm{pH}$ : U.S. Geological Survey Water-Supply Paper 1535-H, 17 p.

Berger, D.L., 1987, Ground-water levels in water years 1984-86 and estimated ground-water pumpage in water years 1984-85, Carson Valley, Douglas County, Nevada: U.S. Geological Survey Open-File Report 86-539, 16 p.

Committee on Water Quality Criteria, 1973, Water quality criteria, 1972: National Academies of Sciences and Engineering, Report EPA-R3-73-033, 594 p.

Cothern, C.R., and Lappenbusch, W.L., 1984, Compliance data for the occurrence of radium and gross $\alpha$-particle activity in drinking water supplies in the United States: Health Physics, v. 46, no. 3 , p. 503-510.

Fairbridge, R.W., ed., 1972, The encyclopedia of geochemistry and environmental sciences, in Encyclopedia of earth sciences series: Stroudsburg, Pa., Dowden, Hutchinson, and Ross, Inc., v. IVA, $1321 \mathrm{p}$.

Feltz, H.R., and Anthony, E.R., eds., 1985, 1985 Water-quality laboratory services catalog: U.S. Geological Survey Open-File Report 84-171, 132 p.

Garcia, K.T., 1989, Ground-water quality in Douglas County, Nevada: U.S. Geological Survey Water-Resources Investigations Report $87-4269,107 \mathrm{p}$.

Governor's Office of Planning Coordination, 1984, Nevada statistical abstract, 1983-84 edition: State of Nevada, 282 p.

Hammond, P.B., and Beliles, R.P., 1980, Metals, in Doull, J., Klaassen, C.D., and Amdur, M.O., eds., Casarett and Doull's toxicology: New York, Macmillan, p. 409-467. 
Hem, J.D., 1985, Study and interpretation of the chemical characteristics of natural waters ( $3 \mathrm{~d}$ ed.): U.S. Geological Survey Water-Supply Paper 2254, 263 p.

Hiltebrand, D.J., Dyksen, J.E, and Raman, Kalyan, 1987, Radon in water supply wells--Treatment facility requirements and costs, in Graves, Barbara, ed., Radon, radium and other radioactivity in ground water: Chelsea, Mich., Lewis Publishers, p. 521-534.

Hobbs, C.H., and McClellan, R.O., 1980, Radiation and radioactive materials, in Doul1, J., Klaassen, C.D., and Amdur, M.O., eds., Casarett and Doull's toxicology: New York, Macmillan, p. 497-530.

Horton, T.R., 1983, Methods and results of EPA's study of radon in drinking water: U.S. Environmental Protection Agency, Office of Radiation Programs, Report EPA-520/5-83-027, 25 p.

Maurer, D.K., 1986, Geohydrology and simulated response to groundwater pumpage in Carson Valley, a river-dominated basin in Douglas County, Nevada, and Alpine County, California: U.S. Geological Survey Water-Resources Investigations Report 86-4328, $109 \mathrm{p}$.

Nevada Bureau of Consumer Health Protection Services, 1980, Water supply regulations, Part I. Water quality standards--monitoring, recordkeeping, and reporting: Nevada Bureau of Consumer Health Protection Services report, $17 \mathrm{p}$.

Nowlin, J.0., 1982, Ground-water levels and water quality in an area near Topaz Lake, Douglas County, Nevada: U.S. Geological Survey Open-File Report 80-2046, 76 p.

Otton, J.K., Zielinski, R.A., and Been, J.M., 1985, Uranium in Holocene valley-fill sediments, and uranium, radon, and helium in waters, Lake Tahoe-Carson Range area, Nevada and California: U.S. Geological Survey Open-File Report 85-389, 30 p.

Rush, F.E., 1968, Index of hydrographic areas in Nevada: Nevada Division of Water Resources, Information Report 6, 38 p.

Skougstad, M.W., Fishman, N.J., Friedman, L.C., Erdmann, D.E., and Duncan, S.S., eds., 1979, Methods for determination of inorganic substances in water and fluvial sediments: U.S. Geological Survey Techniques of Water-Resources Investigations, Book 5, Chapter A1, 626 p. 
Smales, T.J., and Harrill, J.R., 1971, Estimated water use in Nevada: Nevada Division of Water Resources, Water for Nevada Report 2, 32 p.

Thurman, E.M., 1985, Organic geochemistry of natural waters: Dordrecht, The Netherlands, Martinus Nijhoff/Dr W. Junk Publishers, 497 p.

U.S. Bureau of the Census, 1983, 1980 Census of population, number of inhabitants: v. 1, chap. A, pt. 1, United States Summary, 269 p.

U.S. Environmental Protection Agency, 1972, Handbook for analytical quality control in water and wastewater laboratories: Cincinnati, Analytical Quality Control Laboratory, National Environmental Research Center, 99 p.

...-1976, Quality criteria for water: U.S. Environmental Protection Agency report, $256 \mathrm{p}$.

-..-1977, National interim primary drinking water regulations: Office of Water Supply, Report EPA-570/9-76-003, 159 p.

-..-1986a, A citizen's guide to radon: Office of Air and Radiation, Report OPA-86-004, 13 p.

-..-1986b, Quality criteria for water, 1986: Office of Water Regulations and Standards, Report EPA-440/5-86-001, unpaginated.

Wigget, Gail, and Alfors, John, 1986, Selenium: California Geology, May, p. 99-107. 